\title{
Non-Arbitrage Models of Financial Markets
}

Abstract- In the first part of the paper, we construct the models of the complete non-arbitrage financial markets for a wide class of evolutions of risky assets. This construction is based on the observation that for a certain class of risky asset evolutions the martingale measure is invariant with respect to these evolutions. For such a financial market model the only martingale measure being equivalent to an initial measure is built. On such a financial market, formulas for the fair price of contingent liabilities are presented. A multi-parameter model of the financial market is proposed, the martingale measure of which does not depend on the parameters of the model of the evolution of risky assets and is the only one.

Keywords: random process; spot set of measures; optional doob decomposition; supermartingale; martingale; assessment of derivatives; non-arbitrage markets.

\section{GJSFR-A Classification: FOR Code: 240201}

Strictly as per the compliance and regulations of:

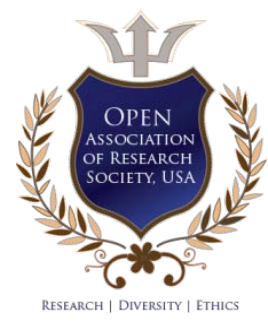

(c) 2021. N. S. Gonchar. This research/review article is distributed under the terms of the Attribution-NonCommercialNoDerivatives 4.0 International (CC BY-NC-ND 4.0). You must give appropriate credit to authors and reference this article if parts of the article are reproduced in any manner. Applicable licensing terms are at https://creativecommons.org/licenses/by-nc$\mathrm{nd} / 4.0 \%$. 


\title{
Non-Arbitrage Models of Financial Markets
}

\author{
N. S. Gonchar
}

Abstract- In the first part of the paper, we construct the models of the complete non-arbitrage financial markets for a wide class of evolutions of risky assets. This construction is based on the observation that for a certain class of risky asset evolutions the martingale measure is invariant with respect to these evolutions. For such a financial market model the only martingale measure being equivalent to an initial measure is built. On such a financial market, formulas for the fair price of contingent liabilities are presented. A multiparameter model of the financial market is proposed, the martingale measure of which does not depend on the parameters of the model of the evolution of risky assets and is the only one.

In the second part of the paper, a model of an incomplete non-arbitrage financial market is proposed. As in the first part of the paper, we use the fact that the family of spot martingale measures is invariant with respect to a certain class of evolutions of risky assets. The set of all martingale measures being equivalent to an initial measure is completely described. Each martingale measure is a linear convex combination of the finite number of spot measures whose structure is completely described. For a wide class of models for the evolution of risky assets, a formula is found for the fair price of a super-hedge, as well as an interval of non-arbitrage prices for any contingent liability. A multi-parameter model of the incomplete financial market is proposed, the martingale measures of which do not depend on the parameters of the model of the evolution of risky assets. For the parameters of the models of the evolution of risky assets, statistical estimates are found for both complete and incomplete non-arbitrage markets.

Keywords: random process; spot set of measures; optional doob decomposition; supermartingale; martingale; assessment of derivatives; non-arbitrage markets.

\section{INTRODUCTION}

In this paper, models of non-arbitrage markets are constructed on the basis of the invariance of a set of spot measures with respect to a certain class of evolution of risky assets. In the first part of the paper, models of complete non arbitrage markets are built on the basis of an analysis of conditions under which there is only one martingale measure. In the second part of the work, models of incomplete nonarbitrage realistic market models are built based on the same principles as in the first part of the work. For the introduced parametric models of the markets, estimates of parameters were obtained based on the observed real values of the evolution of risky assets. This opens up wide opportunities for hedging risks.

Historically the first model evolution of risky assets was suggested in Bachelier's work [4]. Then, in the famous works of Black F. and Scholes M. [5] and Merton R. S. [6] the formula was found for the fair price of the standard call option of European type. The absence of arbitrage in the financial market has a very transparent economic sense, since it can be considered reasonably arranged. The concept of non arbitrage in financial market is associated with the fact that one cannot earn money without risking, that is, to make money you need to invest in risky or risk-free assets. The exact mathematical substantiation of the concept of non arbitrage was first made in the papers [7], [8] for the finite probability space and in the general case in the paper [9]. In the continuous time evolution of risky asset, the proof of absence of arbitrage possibility see in [11]. The value of the established Theorems is that they make it possible to value assets. They got a special name "The First and The Second Fundamental Asset Pricing Theorems." Generalizations of these Theorems are contained in papers [12], [13], [14].

Author: Bogolyubov Institute for Theoretical Physics of NAS of Ukraine. e-mail: mhonchar@i.ua

\footnotetext{
${ }^{1}$ This work was partially supported by the Program of Fundamental Research of the Department of Physics and Astronomy of the National Academy of Sciences of Ukraine (project No 0117U000240).
} 
This work is a continuation of the works [1], [19], [20], [21]. In paper [1], a new method for constructing and describing a family of martingale measures was proposed. This made it possible to build models of non-arbitrage markets. The construction of a realistic model of non-arbitrage markets has been an urgent problem since the moment when the concept of the absence of arbitrage appeared in the scientific literature as the most equitable model of the functioning of financial markets. What could be more attractive than a realistic model that can be built on the basis of observations of the evolution of the financial market. The main obstacle to this was the limited possibilities of constructing a risk-neutral martingale measure for a given evolution of risky assets in the case of a complete market and their complete description in the case of incomplete markets. In the case of discrete evolution of risky assets, the theoretical possibility of the existence of such non-arbitrage markets was established in [7], [8], [9], [10], [11], [12], [13], [14]. But, there were no practically regular methods for constructing such non-arbitrage market models, although such attempts were made for some kind of models of the evolution of risky assets [13], [14]. With the appearance of the work [1], which proposes a regular method for describing all martingale measures for a wide class of evolutions of risky assets [22], [23], [24] that capture the phenomenon of price memory and clustering, it became possible to construct realistic models of non-arbitrage markets. Note that such efforts have been made in this direction, and more about this can be found in the monograph [13], [14]. Valuable is the fact that there is a wide range of models for the evolution of risky assets for which it is possible to build parametric models of non-arbitrage markets whose parameters can be estimated based on statistical data. Problems of risk estimates was considered in papers [15], [16], [17], [18].

This work is the first step in constructing parametric models of non-arbitrage markets whose parameters can be estimated based on empirical data. In this paper, models of the evolution of risk assets on a discrete probabilistic space are considered. Such models can be used to approximate realistic models of the evolution of risky assets. The value of this model is that in this case the structure of the set of martingale measures is relatively simple.

In the case of incomplete non-arbitrage markets, the set of equivalent martingale measures has the cardinality of the continuum, but since they are a linear convex combination of a set of spot measures whose number is finite, this allows calculating the required characteristics using a finite number of operations. This allows a computer to be used to simulate non-arbitrage markets.

In the third section of the work, the necessary and sufficient conditions for the uniqueness of a martingale measure are established in terms of the law of evolution of risky assets, and the only martingale measure is found. Using the results of Section 3 in Section 4, a multi-parameter model of the complete financial market is built and parameter estimates are obtained through empirical data of the financial market. This will allow the model to be adapted to realistic financial markets to estimate the fair price of European-type derivatives with different payment functions.

Section 5 establishes the general structure of the family of equivalent martingale measures for a wide class of risky asset evolutions. The structure of spot measures is completely described, the formulas for the fair price of the super hedge and the range of non-arbitrage prices are established. Based on the results of Section 5, Section 6 builds a multi-parameter model of the incomplete non-arbitrage market. The estimates of the parameters of the model are obtained through empirical observations of the financial market. This will allow the computer to be used to model the financial market.

\section{Evolutions of Risky Assets}

In this section, a class of evolutions of risky assets is described which is used in this paper. This class is fairly wide and includes well known in the literature evolutions of risky assets. Let $\left\{\Omega_{N}, \mathcal{F}_{N}, P_{N}\right\}$ be a direct product of the probability spaces $\left\{\Omega_{i}^{0}, \mathcal{F}_{i}^{0}, P_{i}^{0}\right\}, \quad i=\overline{1, N}, \Omega_{N}=\prod_{i=1}^{N} \Omega_{i}^{0}, P_{N}=\prod_{i=1}^{N} P_{i}^{0}, \mathcal{F}_{N}=\prod_{i=1}^{N} \mathcal{F}_{i}^{0}$, where the $\sigma-$ 
$\operatorname{algebra} \mathcal{F}_{N}$ is a minimal $\sigma$-algebra, generated by the sets $\prod_{i=1}^{N} G_{i}, G_{i} \in \mathcal{F}_{i}^{0}$. On the measurable space $\left\{\Omega_{N}, \mathcal{F}_{N}\right\}$, under the filtration $\mathcal{F}_{n}, n=\overline{1, N}$, we understand the minimal $\sigma$-algebra generated by the sets $\prod_{i=1}^{N} G_{i}, G_{i} \in \mathcal{F}_{i}^{0}$, where $G_{i}=\Omega_{i}^{0}$ for $i>n$. We also introduce the probability spaces $\left\{\Omega_{n}, \mathcal{F}_{n}, P_{n}\right\}, n=\overline{1, N}$, where $\Omega_{n}=\prod_{i=1}^{n} \Omega_{i}^{0}$, $\mathcal{F}_{n}=\prod_{i=1}^{n} \mathcal{F}_{i}^{0}, P_{n}=\prod_{i=1}^{n} P_{i}^{0}$. There is a one-to-one correspondence between the sets of the $\sigma$-algebra $\mathcal{F}_{n}$, belonging to the introduced filtration, and the sets of the $\sigma$ algebra $\mathcal{F}_{n}=\prod_{i=1}^{n} \mathcal{F}_{i}^{0}$ of the measurable space $\left\{\Omega_{n}, \mathcal{F}_{n}\right\}, n=\overline{1, N}$. Therefore, we don't introduce new denotation for the $\sigma$-algebra $\mathcal{F}_{n}$ of the measurable space $\left\{\Omega_{n}, \mathcal{F}_{n}\right\}$, since it always will be clear the difference between the above introduced $\sigma$-algebra $\mathcal{F}_{n}$ of filtration on the measurable space $\left\{\Omega_{N}, \mathcal{F}_{N}\right\}$ and the $\sigma$-algebra $\mathcal{F}_{n}$ of the measurable space $\left\{\Omega_{n}, \mathcal{F}_{n}\right\}, n=\overline{1, N}$.

We assume that the evolution of risky asset $\left\{S_{n}\right\}_{n=0}^{N}$, given on the probability space $\left\{\Omega_{N}, \mathcal{F}_{N}, P_{N}\right\}$, is consistent with the filtration $\mathcal{F}_{n}$, that is, $S_{n}$ is a $\mathcal{F}_{n^{-}}$ measurable. Due to the above one-to-one correspondence between the sets of the $\sigma$-algebra $\mathcal{F}_{n}$, belonging to the introduced filtration, and the sets of the $\sigma$-algebra $\mathcal{F}_{n}$ of the measurable space $\left\{\Omega_{n}, \mathcal{F}_{n}\right\}, n=\overline{1, N}$, we give the evolution of risky assets in the form $\left\{S_{n}\left(\omega_{1}, \ldots, \omega_{n}\right)\right\}_{n=0}^{N}$, where $S_{n}\left(\omega_{1}, \ldots, \omega_{n}\right)$ is an $\mathcal{F}_{n}$-measurable random variable, given on the measurable space $\left\{\Omega_{n}, \mathcal{F}_{n}\right\}$. It is evident that such evolution is consistent with the filtration $\mathcal{F}_{n}$ on the measurable space $\left\{\Omega_{N}, \mathcal{F}_{N}, P_{N}\right\}$.

Further, we assume that

$$
\begin{gathered}
P_{n}\left(\left(\omega_{1}, \ldots, \omega_{n}\right) \in \Omega_{n}, \Delta S_{n}>0\right)>0, \\
P_{n}\left(\left(\omega_{1}, \ldots, \omega_{n}\right) \in \Omega_{n}, \Delta S_{n}<0\right)>0, \quad n=\overline{1, N},
\end{gathered}
$$

where $\Delta S_{n}=S_{n}\left(\omega_{1}, \ldots, \omega_{n}\right)-S_{n-1}\left(\omega_{1}, \ldots, \omega_{n-1}\right), n=\overline{1, N}$.

Let us introduce the denotations

$$
\begin{gathered}
\Omega_{n}^{-}=\left\{\left(\omega_{1}, \ldots, \omega_{n}\right) \in \Omega_{n}, \Delta S_{n} \leq 0\right\}, \quad \Omega_{n}^{+}=\left\{\left(\omega_{1}, \ldots, \omega_{n}\right) \in \Omega_{n}, \Delta S_{n}>0\right\} \\
\Delta S_{n}^{-}=-\Delta S_{n} \chi_{\Omega_{n}^{-}}\left(\omega_{1}, \ldots, \omega_{n}\right), \quad \Delta S_{n}^{+}=\Delta S_{n} \chi_{\Omega_{n}^{+}}\left(\omega_{1}, \ldots, \omega_{n}\right), \\
V_{n}\left(\omega_{1}, \ldots, \omega_{n-1}, \omega_{n}^{1}, \omega_{n}^{2}\right)=\Delta S_{n}^{-}\left(\omega_{1}, \ldots, \omega_{n-1}, \omega_{n}^{1}\right)+\Delta S_{n}^{+}\left(\omega_{1}, \ldots, \omega_{n-1}, \omega_{n}^{2}\right), \\
\left(\omega_{1}, \ldots, \omega_{n-1}, \omega_{n}^{1}\right) \in \Omega_{n}^{-}, \quad\left(\omega_{1}, \ldots, \omega_{n-1}, \omega_{n}^{2}\right) \in \Omega_{n}^{+} .
\end{gathered}
$$

In this paper we assume that

$$
\Omega_{n}^{+}=\Omega_{n-1} \times \Omega_{n}^{0+}, \Omega_{n}^{-}=\Omega_{n-1} \times \Omega_{n}^{0-}, \Omega_{n}^{0+}, \Omega_{n}^{0-} \in \mathcal{F}_{n}^{0}, \Omega_{n}^{0-} \cup \Omega_{n}^{0+}=\Omega_{n}^{0} .
$$

Further, in this paper, we assume that $P_{n}^{0}\left(\Omega_{n}^{0-}\right)>0, P_{n}^{0}\left(\Omega_{n}^{0+}\right)>0, n=\overline{1, N}$. We also assume some technical suppositions: there exist subsets $B_{n, i}^{0-} \in \mathcal{F}_{n}^{0}, \quad i=$ $\overline{1, I_{n}}, I_{n}>1$, and $B_{n, s}^{0+} \in \mathcal{F}_{n}^{0}, s=\overline{1, S_{n}}, S_{n}>1$, satisfying the conditions

$$
B_{n, i}^{0-} \cap B_{n, j}^{0-}=\emptyset, i \neq j, \quad B_{n, s}^{0+} \cap B_{n, l}^{0+}=\emptyset, s \neq l, \quad n=\overline{1, N},
$$




$$
\begin{gathered}
P_{n}^{0}\left(B_{n, i}^{0-}\right)>0, i=\overline{1, I_{n}}, P_{n}^{0}\left(B_{n, s}^{0+}\right)>0, s=\overline{1, S_{n}}, \quad n=\overline{1, N}, \\
\Omega_{n}^{0-}=\bigcup_{i=1}^{I_{n}} B_{n, i}^{0-}, \quad \Omega_{n}^{0+}=\bigcup_{s=1}^{S_{n}} B_{n, s}^{0+}, \quad n=\overline{1, N} .
\end{gathered}
$$

Below, we give the examples of evolutions $\left\{S_{n}\left(\omega_{1}, \ldots, \omega_{n}\right)\right\}_{n=1}^{N}$ for which the representations (5) are true.

Suppose that the random values $a_{i}\left(\omega_{1}, \ldots, \omega_{i}\right), \eta_{i}\left(\omega_{i}\right)$ satisfy the inequalities

$$
a_{i}\left(\omega_{1}, \ldots, \omega_{i}\right)>0, \quad \sup _{\left\{\omega_{1}, \ldots, \omega_{i}\right\} \in \Omega_{i}} a_{i}\left(\omega_{1}, \ldots, \omega_{i}\right)<\frac{1}{\sup _{\omega_{i} \in \Omega_{i}^{0}, \eta_{i}\left(\omega_{i}\right)<0} \eta_{i}^{-}\left(\omega_{i}\right)},
$$

$$
P_{i}^{0}\left(\eta_{i}\left(\omega_{i}\right)<0\right)>0, \quad P_{i}^{0}\left(\eta_{i}\left(\omega_{i}\right)>0\right)>0, \quad i=\overline{1, N}
$$

If $S_{n}\left(\omega_{1}, \ldots, \omega_{n}\right)$ is given by the formula

$$
S_{n}\left(\omega_{1}, \ldots, \omega_{n}\right)=S_{0} \prod_{i=1}^{n}\left(1+a_{i}\left(\omega_{1}, \ldots, \omega_{i}\right) \eta_{i}\left(\omega_{i}\right)\right), \quad n=\overline{1, N}
$$

then

$$
\begin{gathered}
\left\{\omega_{i} \in \Omega_{i}^{0}, \eta_{i}\left(\omega_{i}\right) \leq 0\right\}=\Omega_{i}^{0-}, \quad\left\{\omega_{i} \in \Omega_{i}^{0}, \eta_{i}\left(\omega_{i}\right)>0\right\}=\Omega_{i}^{0+}, \\
\Omega_{i}^{-}=\Omega_{i-1} \times \Omega_{i}^{0-}, \quad \Omega_{i}^{+}=\Omega_{i-1} \times \Omega_{i}^{0+}, \quad i=\overline{1, N} .
\end{gathered}
$$

Let us note that not only the evolutions given by the formula (8) provide the representation (5). In this work, we use the evolutions of the kind (8). Below we give examples of the evolution of risky assets that have the form (8). For example, if

$$
S_{n}\left(\omega_{1}, \ldots, \omega_{n}\right)=S_{0} \prod_{i=1}^{n} e^{\sigma_{i}\left(\omega_{1}, \ldots, \omega_{i-1}\right) \varepsilon_{i}\left(\omega_{i}\right)}, \quad n=\overline{1, N}
$$

where the random values $\sigma_{i}\left(\omega_{1}, \ldots, \omega_{i-1}\right) \geq \sigma_{i}^{0}>0, i=\overline{1, N}$, and $P_{i}^{0}\left(\varepsilon_{i}\left(\omega_{i}\right)<0\right)>$ $0, P_{i}^{0}\left(\varepsilon_{i}\left(\omega_{i}\right)>0\right)>0$, then such an evolution has the form (8) with

$$
a_{i}\left(\omega_{1}, \ldots, \omega_{i}\right)=\frac{e^{\sigma_{i}\left(\omega_{1}, \ldots, \omega_{i-1}\right) \varepsilon_{i}\left(\omega_{i}\right)}-1}{e^{\sigma_{i}^{0} \varepsilon_{i}\left(\omega_{i}\right)}-1}, \quad \eta_{i}\left(\omega_{i}\right)=e^{\sigma_{i}^{0} \varepsilon_{i}\left(\omega_{i}\right)}-1, \quad i=\overline{1, N} .
$$

satisfying needed conditions.

\section{iII. Uniqueness of the Martingale Measure}

In this section, the necessary and sufficient conditions in terms of the evolution of risky assets are obtained relative to the uniqueness of martingale measure. Under the fairly wide assumptions about the evolution of risky assets, an expression for a single martingale measure is found. Based on the explicit construction of the martingale measure and its invariance with respect to a certain type of evolutions, it is possible to construct the models of non arbitrage markets, both complete and incomplete. 
In this and section 4 , we put that $\Omega_{i}^{0}=\left\{\omega_{i}^{1}, \omega_{i}^{2}\right\}$. Denote by $\mathcal{F}_{i}^{0}$ the $\sigma$-algebra of all subsets of the set $\Omega_{i}^{0}$. Let $P_{i}^{0}$ be a probability measure on $\mathcal{F}_{i}^{0}$. We assume that $P_{i}^{0}\left(\omega_{i}^{s}\right)>0, i=\overline{1, N}, s=\overline{1,2}$. As before, we put that the probability space $\left\{\Omega_{N}, \mathcal{F}_{N}, P_{N}\right\}$ is a direct product of the probability spaces $\left\{\Omega_{i}^{0}, \mathcal{F}_{i}^{0}, P_{i}^{0}\right\}, i=\overline{1, N}$, and we put $N<\infty$. We also consider the probability spaces $\left\{\Omega_{n}, \mathcal{F}_{n}, P_{n}\right\}, n=\overline{1, N}$, being the direct product of the probability spaces $\left\{\Omega_{i}^{0}, \mathcal{F}_{i}^{0}, P_{i}^{0}\right\}, i=\overline{1, n}$. We assume that the evolution of a risky asset is given by the formula

$$
\begin{gathered}
S_{n}\left(\omega_{1}, \ldots, \omega_{n}\right)= \\
S_{0} \prod_{i=1}^{n}\left(1+a_{i}\left(\omega_{1}, \ldots, \omega_{i}\right) \eta_{i}\left(\omega_{i}\right)\right),\left\{\omega_{1}, \ldots, \omega_{n-1}, \omega_{n}\right\} \in \Omega_{n}, n=\overline{1, N},
\end{gathered}
$$

where the random values $a_{n}\left(\omega_{1}, \ldots, \omega_{n-1}, \omega_{n}\right), \eta_{n}\left(\omega_{n}\right), \quad n=\overline{1, N}$, given on the probability space $\left\{\Omega_{n}, \mathcal{F}_{n}, P_{n}\right\}$, satisfy the conditions

$$
\begin{gathered}
a_{n}\left(\omega_{1}, \ldots, \omega_{n-1}, \omega_{n}\right)>0, \quad \max _{\left\{\omega_{1}, \quad \ldots, \omega_{n-1}\right\} \in \Omega_{n-1}} a_{n}\left(\omega_{1}, \ldots, \omega_{n-1}, \omega_{n}^{1}\right)<\frac{1}{\eta_{n}^{-}\left(\omega_{n}^{1}\right)}, \\
\eta_{n}\left(\omega_{n}^{2}\right)>0, \quad \eta_{n}\left(\omega_{n}^{1}\right)<0 .
\end{gathered}
$$

So, for $\Delta S_{n}\left(\omega_{1}, \ldots, \omega_{n-1}, \omega_{n}\right), n=\overline{1, N}$, the representation

$$
\begin{gathered}
\Delta S_{n}\left(\omega_{1}, \ldots, \omega_{n-1}, \omega_{n}\right)= \\
S_{n-1}\left(\omega_{1}, \ldots, \omega_{n-1}\right) a_{n}\left(\omega_{1}, \ldots, \omega_{n-1}, \omega_{n}\right) \eta_{n}\left(\omega_{n}\right)= \\
d_{n}\left(\omega_{1}, \ldots, \omega_{n-1}, \omega_{n}\right) \eta_{n}\left(\omega_{n}\right), \quad d_{n}\left(\omega_{1}, \ldots, \omega_{n-1}, \omega_{n}\right)>0, \quad n=\overline{1, N}, \quad S_{0}>0,
\end{gathered}
$$

is true, From these conditions, we obtain $\Omega_{n}^{-}=\Omega_{n-1} \times \Omega_{n}^{0-}, \Omega_{n}^{+}=\Omega_{n-1} \times \Omega_{n}^{0+}$, where $\Omega_{n}^{0-}=\left\{\omega_{n} \in \Omega_{n}^{0}, \eta_{n}\left(\omega_{n}\right) \leq 0\right\}, \Omega_{n}^{0+}=\left\{\omega_{n} \in \Omega_{n}^{0}, \eta_{n}\left(\omega_{n}\right)>0\right\}$.

From the suppositions above, it follows that $P_{n}^{0}\left(\Omega_{n}^{0-}\right)>0, P_{n}^{0}\left(\Omega_{n}^{0+}\right)>0$. The measure $P_{n}^{0-}$ is a contraction of the measure $P_{n}^{0}$ on the $\sigma$-algebra $\mathcal{F}_{n}^{0-}=\Omega_{n}^{0-} \cap \mathcal{F}_{n}^{0}$, $P_{n}^{0+}$ is a contraction of the measure $P_{n}^{0}$ on the $\sigma$-algebra $\mathcal{F}_{n}^{0+}=\Omega_{n}^{0+} \cap \mathcal{F}_{n}^{0}$.

Let us introduce the following denotation. For every point $\left\{\omega_{1}, \ldots, \omega_{n-1}, \omega_{n}\right\} \in$ $\Omega_{n}$, we introduce the set $A\left(\omega_{1}, \ldots, \omega_{n-1}, \omega_{n}\right) \in \Omega_{N}$, where

$$
A\left(\omega_{1}, \ldots, \omega_{n-1}, \omega_{n}\right)=\bigcup_{i_{n+1}=1, \ldots, i_{N}=1}^{2}\left\{\omega_{1}, \ldots, \omega_{n-1}, \omega_{n}, \omega_{n+1}^{i_{n+1}}, \ldots, \omega_{N}^{i_{N}}\right\} .
$$

For fixed indexes $i_{1}, \ldots, i_{n}$ we also use the denotation

$$
A\left(\omega_{1}^{i_{1}}, \ldots, \omega_{n-1}^{i_{n-1}}, \omega_{n}^{i_{n}}\right)=A^{i_{1}, \ldots, i_{n}} .
$$

It is evident that every set $A^{i_{1}, \ldots, i_{n}}$ has the form

$$
A^{i_{1}, \ldots, i_{n}}=\bigcup_{i_{n+1}=1, \ldots, i_{N}=1}^{2}\left\{\omega_{1}^{i_{1}}, \ldots, \omega_{n}^{i_{n}}, \omega_{n+1}^{i_{n+1}}, \ldots, \omega_{N}^{i_{N}}\right\}
$$


where indexes $i_{s}$ takes only one value from the set $\{1,2\}$. Then, $A^{i_{1}, \ldots, i_{n-1}}=$ $A^{i_{1}, \ldots, i_{n-1}, 1} \cup A^{i_{1}, \ldots, i_{n-1}, 2} \in \mathcal{F}_{n-1}$, where

$$
\begin{aligned}
A^{i_{1}, \ldots, i_{n-1}, 1} & =\bigcup_{i_{n+1}=1, \ldots, i_{N}=1}^{2}\left\{\omega_{1}^{i_{1}}, \ldots, \omega_{n-1}^{i_{n-1}}, \omega_{n}^{1}, \omega_{n+1}^{i_{n+1}}, \ldots, \omega_{N}^{i_{N}}\right\} \in \mathcal{F}_{n} \\
A^{i_{1}, \ldots, i_{n-1}, 2} & \left.=\bigcup_{i_{n+1}=1, \ldots, i_{N}=1}^{2}\left\{\omega_{1}^{i_{1}}, \ldots, \omega_{n-1}^{i_{n-1}}, \omega_{n}^{2}, \omega_{n+1}^{i_{n+1}}, \ldots, \omega_{N}^{i_{N}}\right\}\right\} \in \mathcal{F}_{n} .
\end{aligned}
$$

If $P_{N}$ is a measure on $\mathcal{F}_{N}$, then

$$
P_{N}\left(A\left(\omega_{1}, \ldots, \omega_{n-1}, \omega_{n}\right)\right)=\sum_{i_{n+1}=1, \ldots, i_{N}=1}^{2} P_{N}\left(\left\{\omega_{1}, \ldots, \omega_{n-1}, \omega_{n}, \omega_{n+1}^{i_{n+1}}, \ldots, \omega_{N}^{i_{N}}\right\}\right) .
$$

We give an evident construction of martingale measure for risky assets evolution, given by the formula (11). Below, we assume that measures $P_{n}^{0}$ is concentrated at points $\omega_{n}^{1}, \omega_{n}^{2} \in \Omega_{n}^{0}$, where $\omega_{n}^{1} \in \Omega_{n}^{0-}, \omega_{n}^{2} \in \Omega_{n}^{0+}$ and we have the representation $\Omega_{n}^{-}=\Omega_{n-1} \times \Omega^{0-}$ and $\Omega_{n}^{+}=\Omega_{n-1} \times \Omega^{0+}$. So, we have $\eta_{n}\left(\omega_{n}^{1}\right)<0, \eta_{n}\left(\omega_{n}^{2}\right)>0$.

Let us put $P_{n}^{0}\left(\omega_{n}^{1}\right)=p_{n}, P_{n}^{0}\left(\omega_{n}^{2}\right)=1-p_{n}$, where $0<p_{n}<1$. Then, to satisfy the conditions $(14-16)$, (see [1]) we need to put

$$
\alpha_{n}\left(\left\{\omega_{1}^{1}, \ldots, \omega_{n}^{1}\right\} ;\left\{\omega_{1}^{2}, \ldots, \omega_{n}^{2}\right\}\right)=\frac{1}{p_{n}\left(1-p_{n}\right)}, \quad n=\overline{1, N}
$$

and to require that

$$
\begin{aligned}
& \Delta S_{n}^{-}\left(\omega_{1}, \ldots, \omega_{n-1}, \omega_{n}^{1}\right)<\infty, \quad\left(\omega_{1}, \ldots, \omega_{n-1}, \omega_{n}^{1}\right) \in \Omega_{n}^{-}, \\
& \Delta S_{n}^{+}\left(\omega_{1}, \ldots, \omega_{n-1}, \omega_{n}^{2}\right)<\infty, \quad\left(\omega_{1}, \ldots, \omega_{n-1}, \omega_{n}^{2}\right) \in \Omega_{n}^{+} .
\end{aligned}
$$

The next Lemma 1 is a consequence of results in [1].

Lemma 1. On the probability space $\left\{\Omega_{N}, \mathcal{F}_{N}, P_{N}\right\}$, being the direct product of the probability spaces $\left\{\Omega_{i}^{0}, \mathcal{F}_{i}^{0}, P_{i}^{0}\right\}$, for the evolution of risky asset given by the formula (11) only one spot measure $\mu_{\left\{\omega_{1}^{1}, \omega_{1}^{2}\right\}, \ldots,\left\{\omega_{N}^{1}, \omega_{N}^{2}\right\}}(A)$ exists, where $\left\{\omega_{i}^{1}, \omega_{i}^{2}\right\} \in \Omega_{i}^{0}, \quad i=$ $\overline{1, N}$. For it the representation

$$
\begin{gathered}
\mu_{0}(A)=\mu_{\left\{\omega_{1}^{1}, \omega_{1}^{2}\right\}, \ldots,\left\{\omega_{N}^{1}, \omega_{N}^{2}\right\}}(A)= \\
\sum_{i_{1}=1}^{2} \ldots \sum_{i_{N}=1}^{2} \prod_{n=1}^{N} \psi_{n}\left(\omega_{1}^{i_{1}}, \ldots, \omega_{n}^{i_{n}}\right) \chi_{A}\left(\omega_{1}^{i_{1}}, \ldots, \omega_{N}^{i_{N}}\right), \quad A \in \mathcal{F}_{N},
\end{gathered}
$$

is true. This measure is martingale measure for the considered evolution of risky asset, where

$$
\begin{gathered}
\psi_{n}\left(\omega_{1}, \ldots, \omega_{n}\right)=\chi_{\Omega_{n}^{-}}\left(\omega_{1}, \ldots, \omega_{n-1}, \omega_{n}\right) \psi_{n}^{1}\left(\omega_{1}, \ldots, \omega_{n}\right)+ \\
\chi_{\Omega_{n}^{+}}\left(\omega_{1}, \ldots, \omega_{n-1}, \omega_{n}\right) \psi_{n}^{2}\left(\omega_{1}, \ldots, \omega_{n}\right)
\end{gathered}
$$




$$
\begin{gathered}
\psi_{n}^{1}\left(\omega_{1}, \ldots, \omega_{n-1}, \omega_{n}\right)= \\
\frac{\Delta S_{n}^{+}\left(\omega_{1}, \ldots, \omega_{n-1}, \omega_{n}^{2}\right)}{V_{n}\left(\omega_{1}, \ldots, \omega_{n-1}, \omega_{n}^{1}, \omega_{n}^{2}\right)}, \quad\left(\omega_{1}, \ldots, \omega_{n-1}\right) \in \Omega_{n-1}, \\
\psi_{n}^{2}\left(\omega_{1}, \ldots, \omega_{n-1}, \omega_{n}\right)= \\
\frac{\Delta S_{n}^{-}\left(\omega_{1}, \ldots, \omega_{n-1}, \omega_{n}^{1}\right)}{V_{n}\left(\omega_{1}, \ldots, \omega_{n-1}, \omega_{n}^{1}, \omega_{n}^{2}\right)}, \quad\left(\omega_{1}, \ldots, \omega_{n-1}\right) \in \Omega_{n-1} .
\end{gathered}
$$

Next Theorem 1 appeared first in [2] (Theorem 1.4.1), where it was proved under the less general conditions.

Theorem 1. On the probability space $\left\{\Omega_{N}, \mathcal{F}_{N}, P_{N}\right\}$, being the direct product of the probability spaces $\left\{\Omega_{i}^{0}, \mathcal{F}_{i}^{0}, P_{i}^{0}\right\}$, suppose that the evolution of risky asset $\left\{S_{n}\left(\omega_{1}, \ldots, \omega_{n}\right)\right\}_{n=1}^{N}$ is given by the formula (11). The necessary and sufficient conditions of the uniqueness of martingale measure $\mu_{0}(A), A \in \mathcal{F}_{N}$, are the inequalities

$$
S_{n}\left(\omega_{1}^{i_{1}}, \ldots, \omega_{n-1}^{i_{n-1}}, \omega_{n}^{1}\right) \neq S_{n}\left(\omega_{1}^{i_{1}}, \ldots, \omega_{n-1}^{i_{n-1}}, \omega_{n}^{2}\right), \quad n=\overline{1, N}
$$

for every set of indexes $i_{1}, \ldots, i_{n-1}$. For any martingale $\left\{m_{n}\left(\omega_{1}, \ldots, \omega_{n-1}, \omega_{n}\right)\right\}_{n=0}^{N}$ relative to the unique measure $\mu_{0}(A)$ the representation

$$
\begin{gathered}
m_{n}\left(\omega_{1}, \ldots, \omega_{n-1}, \omega_{n}\right)= \\
\sum_{k=1}^{n} C_{k}\left(\omega_{1}, \ldots, \omega_{k-1}\right)\left[S_{k}\left(\omega_{1}, \ldots, \omega_{k-1}, \omega_{i}\right)-S_{k-1}\left(\omega_{1}, \ldots, \omega_{k-1}\right)\right]+ \\
m_{0}, \quad n=\overline{1, N}
\end{gathered}
$$

is true, where

$$
\begin{gathered}
C_{k}\left(\omega_{1}, \ldots, \omega_{k-1}\right)=\sum_{i_{1}=1, \ldots, i_{k-1}=1}^{2} d_{i_{1}, \ldots, i_{k-1}} \chi_{A^{i_{1}, \ldots, i_{k-1}}}\left(\omega_{1}, \ldots, \omega_{k-1}\right) . \\
d_{i_{1}, \ldots, i_{k-1}}= \\
\frac{m_{k}\left(\omega_{1}^{i_{1}}, \ldots, \omega_{k-1}^{i_{k-1}}, \omega_{k}^{1}\right)-m_{k}\left(\omega_{1}^{i_{1}}, \ldots, \omega_{k-1}^{i_{k-1}}, \omega_{k}^{2}\right)}{S_{k}\left(\omega_{1}^{i_{1}}, \ldots, \omega_{k-1}^{i_{k-1}}, \omega_{k}^{1}\right)-S_{k}\left(\omega_{1}^{i_{1}}, \ldots, \omega_{k-1}^{i_{k-1}}, \omega_{k}^{2}\right)}, \quad k=\overline{1, N} .
\end{gathered}
$$

Proof. The necessity. Suppose that the evolution $\left\{S_{n}\left(\omega_{1}, \ldots, \omega_{n}\right)\right\}_{n=1}^{N}$ of the risky asset on the probability space $\left\{\Omega_{N}, \mathcal{F}_{N}, P_{N}\right\}$ is such that the martingale measure $\mu_{0}(A), A \in \mathcal{F}_{N}$, being equivalent to the measure $P_{N}$, is unique. Then, for every attainable contingent liability $m_{N}\left(\omega_{1}, \ldots, \omega_{N}\right)$ the representation $(21)$ is true [11] for some $\mathcal{F}_{k-1}$-measurable finite valued random value $C_{k}\left(\omega_{1}, \ldots, \omega_{k-1}\right), k=\overline{1, N}$, where $m_{n}\left(\omega_{1}, \ldots, \omega_{n-1}, \omega_{n}\right)=E^{\mu_{0}}\left\{m_{N}\left(\omega_{1}, \ldots, \omega_{N}\right) \mid \mathcal{F}_{n}\right\}$. For $m_{n}\left(\omega_{1}, \ldots, \omega_{n-1}, \omega_{n}\right)$ and $S_{n}\left(\omega_{1}, \ldots, \omega_{n-1}, \omega_{n}\right)$ the representations 


$$
\begin{aligned}
& m_{n}\left(\omega_{1}, \ldots, \omega_{n-1}, \omega_{n}\right)= \\
& \sum_{i_{1}=1, \ldots, i_{n}=1}^{2} \frac{\chi_{A^{i_{1}, \ldots, i_{n-1}, i_{n}}\left(\omega_{1}, \ldots, \omega_{n}\right)}}{\mu_{0}\left(A^{i_{1}, \ldots, i_{n-1}, i_{n}}\right)} \int_{A^{i_{1}, \ldots, i_{n-1}, i_{n}}} m_{N}\left(\omega_{1}, \ldots, \omega_{N}\right) d \mu_{0}, n=\overline{1, N}, \\
& S_{n}\left(\omega_{1}, \ldots, \omega_{n-1}, \omega_{n}\right)= \\
& \sum_{i_{1}=1, \ldots, i_{n}=1}^{2} \frac{\chi_{A^{i_{1}, \ldots, i_{n-1}, i_{n}}\left(\omega_{1}, \ldots, \omega_{n}\right)}}{\mu_{0}\left(A^{i_{1}, \ldots, i_{n-1}, i_{n}}\right)} \int_{A^{i_{1}, \ldots, i_{n-1}, i_{n}}} S_{N}\left(\omega_{1}, \ldots, \omega_{N}\right) d \mu_{0}, n=\overline{1, N}
\end{aligned}
$$

are true. From the representation $(21)$ and the equality $(22)$ for $\left\{\omega_{1}, \ldots, \omega_{n-1}\right\} \in$ $A^{i_{1}, \ldots, i_{n-1}}$ we obtain the equality

$$
\begin{aligned}
& \frac{\chi_{A^{i_{1}, \ldots, i_{n-1}, 1}}\left(\omega_{1}, \ldots, \omega_{n}\right)}{\mu_{0}\left(A^{i_{1}, \ldots, i_{n-1}, 1}\right)} \int_{A^{i_{1}, \ldots, i_{n-1}, 1}} m\left(\omega_{1}, \ldots, \omega_{N}\right) d \mu_{0}+ \\
& \frac{\chi_{A^{i_{1}, \ldots, i_{n-1}, 2}}\left(\omega_{1}, \ldots, \omega_{n}\right)}{\mu_{0}\left(A^{i_{1}, \ldots, i_{n-1}, 2}\right)} \int_{A^{i_{1}, \ldots, i_{n-1}, 2}} m\left(\omega_{1}, \ldots, \omega_{N}\right) d \mu_{0}- \\
& \frac{\chi_{A^{i_{1}, \ldots, i_{n-1}}}\left(\omega_{1}, \ldots, \omega_{n-1}\right)}{\mu_{0}\left(A^{i_{1}, \ldots, i_{n-1}}\right)} \int_{A^{i_{1}, \ldots, i_{n-1}}} m\left(\omega_{1}, \ldots, \omega_{N}\right) d \mu_{0}= \\
& d_{i_{1}, \ldots, i_{n-1}} \chi_{A^{i_{1}, \ldots, i_{n-1}}}\left(\omega_{1}, \ldots, \omega_{n-1}\right) \times \\
& {\left[\frac{\chi_{A^{i_{1}, \ldots, i_{n-1}, 1}}\left(\omega_{1}, \ldots, \omega_{n}\right)}{\mu_{0}\left(A^{i_{1}, \ldots, i_{n-1}, 1}\right)} \int_{A^{i_{1}, \ldots, i_{n-1}, 1}} S_{N}\left(\omega_{1}, \ldots, \omega_{N}\right) d \mu_{0}+\right.} \\
& \frac{\chi_{A^{i_{1}, \ldots, i_{n-1}, 2}\left(\omega_{1}, \ldots, \omega_{n}\right)}}{\mu_{0}\left(A^{i_{1}, \ldots, i_{n-1}, 2}\right)} \int_{A^{i_{1}, \ldots, i_{n-1}, 2}} S_{N}\left(\omega_{1}, \ldots, \omega_{N}\right) d \mu_{0}-
\end{aligned}
$$



where $d_{i_{1}, \ldots, i_{n-1}}$ is finite. Since

$$
\int_{A^{i_{1}, \ldots, i_{n-1}}} m\left(\omega_{1}, \ldots, \omega_{N}\right) d \mu_{0}=
$$




$$
\int_{A^{i_{1}, \ldots, i_{n-1}, 1}} m\left(\omega_{1}, \ldots, \omega_{N}\right) d \mu_{0}+\int_{A^{i_{1}, \ldots, i_{n-1}, 2}} m\left(\omega_{1}, \ldots, \omega_{N}\right) d \mu_{0}
$$

we have

$$
\begin{aligned}
& \mu_{0}\left(A^{i_{1}, \ldots, i_{n-1}}\right) \int_{A^{i_{1}, \ldots, i_{n-1}, 1}} m\left(\omega_{1}, \ldots, \omega_{N}\right) d \mu_{0}- \\
& \mu_{0}\left(A^{i_{1}, \ldots, i_{n-1}, 1}\right) \int_{A^{i_{1}, \ldots, i_{n-1}}} m\left(\omega_{1}, \ldots, \omega_{N}\right) d \mu_{0}= \\
& {\left[\mu_{0}\left(A^{i_{1}, \ldots, i_{n-1}, 1}\right)+\mu_{0}\left(A^{i_{1}, \ldots, i_{n-1}, 2}\right)\right] \int_{A^{i_{1}, \ldots, i_{n-1}, 1}} m\left(\omega_{1}, \ldots, \omega_{N}\right) d \mu_{0}-} \\
& \mu_{0}\left(A^{i_{1}, \ldots, i_{n-1}, 1}\right)\left[\int_{A^{i_{1}, \ldots, i_{n-1}, 1}} m\left(\omega_{1}, \ldots, \omega_{N}\right) d \mu_{0}+\int_{A^{i_{1}, \ldots, i_{n-1}, 2}} m\left(\omega_{1}, \ldots, \omega_{N}\right) d \mu_{0}\right]= \\
& \mu_{0}\left(A^{i_{1}, \ldots, i_{n-1}, 2}\right) \int_{A^{i_{1}, \ldots, i_{n-1}, 1}} m\left(\omega_{1}, \ldots, \omega_{N}\right) d \mu_{0}- \\
& \mu_{0}\left(A^{i_{1}, \ldots, i_{n-1}, 1}\right) \int_{A^{i_{1}, \ldots, i_{n-1}, 2}} m\left(\omega_{1}, \ldots, \omega_{N}\right) d \mu_{0}
\end{aligned}
$$

Further,

$$
\begin{gathered}
\mu_{0}\left(A^{i_{1}, \ldots, i_{n-1}}\right) \int_{A^{i_{1}, \ldots, i_{n-1}, 2}} m\left(\omega_{1}, \ldots, \omega_{N}\right) d \mu_{0}- \\
\mu_{0}\left(A^{i_{1}, \ldots, i_{n-1}, 2}\right) \int_{A^{i_{1}, \ldots, i_{n-1}}} m\left(\omega_{1}, \ldots, \omega_{N}\right) d \mu_{0}= \\
\mu_{0}\left(A^{i_{1}, \ldots, i_{n-1}, 2}\right)\left[A_{\left.\left.\mu^{i_{1}, \ldots, i_{n-1}, 1}\right)+\mu_{0}\left(A^{i_{1}, \ldots, i_{n-1}, 2}\right)\right]} \int_{A^{i_{1}, \ldots, i_{n-1}, 2}} m\left(\omega_{1}, \ldots, \omega_{N}\right) d \mu_{0}-\right. \\
{\left[\int_{A^{i_{1}, \ldots, i_{n-1}, 1}} m\left(\omega_{1}, \ldots, \omega_{N}\right) d \mu_{0}+\int_{A^{i_{1}, \ldots, i_{n-1}, 2}} m\left(\omega_{1}, \ldots, \omega_{N}\right) d \mu_{0}\right]=}
\end{gathered}
$$




$$
\begin{gathered}
-\left[\mu_{0}\left(A^{i_{1}, \ldots, i_{n-1}, 2}\right) \int_{A^{i_{1}, \ldots, i_{n-1}, 1}} m\left(\omega_{1}, \ldots, \omega_{N}\right) d \mu_{0}-\right. \\
\left.\mu_{0}\left(A^{i_{1}, \ldots, i_{n-1}, 1}\right) \int_{A^{i_{1}, \ldots, i_{n-1}, 2}} m\left(\omega_{1}, \ldots, \omega_{N}\right) d \mu_{0}\right] .
\end{gathered}
$$

If to put

$$
\begin{gathered}
R_{1}^{m}\left(\omega_{1}^{i_{1}}, \ldots, \omega_{n-1}^{i_{n-1}}\right)=\mu_{0}\left(A^{i_{1}, \ldots, i_{n-1}, 1}\right) \int_{A^{i_{1}, \ldots, i_{n-1}, 2}} m\left(\omega_{1}, \ldots, \omega_{N}\right) d \mu_{0}- \\
\mu_{0}\left(A^{i_{1}, \ldots, i_{n-1}, 2}\right) \int_{A^{i_{1}, \ldots, i_{n-1}, 1}} m\left(\omega_{1}, \ldots, \omega_{N}\right) d \mu_{0}, \\
R_{1}^{S_{N}\left(\omega_{1}^{i_{1}}, \ldots, \omega_{n-1}^{i_{n-1}}\right)=\mu_{0}\left(A^{i_{1}, \ldots, i_{n-1}, 1}\right)} \int_{A^{i_{1}, \ldots, i_{n-1}, 2}} S_{N}\left(\omega_{1}, \ldots, \omega_{N}\right) d \mu_{0}- \\
\mu_{0}\left(A^{i_{1}, \ldots, i_{n-1}, 2}\right) \int_{A^{i_{1}, \ldots, i_{n-1}, 1}} S_{N}\left(\omega_{1}, \ldots, \omega_{N}\right) d \mu_{0} .
\end{gathered}
$$

Then, the equality (26) is transformed into the equality

$$
R_{1}^{m}\left(\omega_{1}^{i_{1}}, \ldots, \omega_{n-1}^{i_{n-1}}\right)=d_{i_{1}, \ldots, i_{n-1}} R_{1}^{S_{N}}\left(\omega_{1}^{i_{1}}, \ldots, \omega_{n-1}^{i_{n-1}}\right) .
$$

Due to that $S_{n}\left(\omega_{1}, \ldots, \omega_{n}\right)$ and $m_{n}\left(\omega_{1}, \ldots, \omega_{n}\right)$ are martingales relative to the measure $\mu_{0}$ and $A^{i_{1}, \ldots, i_{n-1}, 1}, A^{i_{1}, \ldots, i_{n-1}, 2} \in \mathcal{F}_{n}$ we have

$$
\begin{gathered}
\int_{A^{i_{1}, \ldots, i_{n-1}, 1}} S_{N}\left(\omega_{1}, \ldots, \omega_{N}\right) d \mu_{0}=\int_{A^{i_{1}, \ldots, i_{n-1}, 1}} S_{n}\left(\omega_{1}, \ldots, \omega_{n}\right) d \mu_{0}= \\
\mu_{0}\left(A^{i_{1}, \ldots, i_{n-1}, 1}\right) S_{n}\left(\omega_{1}, \ldots, \omega_{n}^{1}\right), \\
\int_{A^{i_{1}, \ldots, i_{n-1}, 2}} S_{N}\left(\omega_{1}, \ldots, \omega_{N}\right) d \mu_{0}=\int_{A^{i_{1}, \ldots, i_{n-1}, 2}} S_{n}\left(\omega_{1}, \ldots, \omega_{n}\right) d \mu_{0}= \\
\mu_{0}\left(A^{i_{1}, \ldots, i_{n-1}, 2}\right) S_{n}\left(\omega_{1}, \ldots, \omega_{n}^{2}\right), \\
\int_{A^{i_{1}, \ldots, i_{n-1}, 1}} m_{N}\left(\omega_{1}, \ldots, \omega_{N}\right) d \mu_{0}=\int_{A^{i_{1}, \ldots, i_{n-1}, 1}} m_{n}\left(\omega_{1}, \ldots, \omega_{n}\right) d \mu_{0}
\end{gathered}
$$




$$
\begin{gathered}
\mu_{0}\left(A^{i_{1}, \ldots, i_{n-1}, 1}\right) m_{n}\left(\omega_{1}, \ldots, \omega_{n}^{1}\right) \\
\int_{A^{i_{1}, \ldots, i_{n-1}, 2}} m_{N}\left(\omega_{1}, \ldots, \omega_{N}\right) d \mu_{0}=\int_{A^{i_{1}, \ldots, i_{n-1}, 2}} m_{n}\left(\omega_{1}, \ldots, \omega_{n}\right) d \mu_{0}= \\
\mu_{0}\left(A^{i_{1}, \ldots, i_{n-1}, 2}\right) m_{n}\left(\omega_{1}, \ldots, \omega_{n}^{2}\right) .
\end{gathered}
$$

Since $d_{i_{1}, \ldots, i_{n-1}}$ is finite, then $R_{1}^{S_{N}}\left(\omega_{1}^{i_{1}}, \ldots, \omega_{n-1}^{i_{n-1}}\right) \neq 0$. The last means that inequality (20) takes place. This proves the equality

$$
\begin{gathered}
d_{i_{1}, \ldots, i_{n-1}}= \\
\frac{m_{n}\left(\omega_{1}^{i_{1}}, \ldots, \omega_{n-1}^{i_{n-1}}, \omega_{n}^{1}\right)-m_{n}\left(\omega_{1}^{i_{1}}, \ldots, \omega_{n-1}^{i_{n-1}}, \omega_{n}^{2}\right)}{S_{n}\left(\omega_{1}^{i_{1}}, \ldots, \omega_{n-1}^{i_{n-1}}, \omega_{n}^{1}\right)-S_{n}\left(\omega_{1}^{i_{1}}, \ldots, \omega_{n-1}^{i_{n-1}}, \omega_{n}^{2}\right)} \\
n=\overline{1, N}
\end{gathered}
$$

which means that (23) is true, where we introduced the denotation

$$
\begin{gathered}
m_{n}\left(\omega_{1}, \ldots, \omega_{n}\right)=E^{\mu_{0}}\left\{m\left(\omega_{1}, \ldots, \omega_{N}\right) \mid \mathcal{F}_{n}\right\}= \\
\sum_{i_{n+1}=1, \ldots, i_{N}=1}^{2} m\left(\omega_{1}, \ldots, \omega_{n}, \omega_{n}^{i_{n+1}}, \ldots, \omega_{N}^{i_{N}}\right) \mu_{0}\left(\left\{\omega_{1}, \ldots, \omega_{n}, \omega_{n}^{i_{n+1}}, \ldots, \omega_{N}^{i_{N}}\right\}\right), \\
S_{n}\left(\omega_{1}, \ldots, \omega_{n}\right)=E^{\mu_{0}}\left\{S_{N}\left(\omega_{1}, \ldots, \omega_{N}\right) \mid \mathcal{F}_{n}\right\}= \\
\sum_{i_{n+1}=1, \ldots, i_{N}=1}^{2} S_{N}\left(\omega_{1}, \ldots, \omega_{n}, \omega_{n}^{i_{n+1}}, \ldots, \omega_{N}^{i_{N}}\right) \mu_{0}\left(\left\{\omega_{1}, \ldots, \omega_{n}, \omega_{n}^{i_{n+1}}, \ldots, \omega_{N}^{i_{N}}\right\}\right) .
\end{gathered}
$$

This proves the necessity.

Proof of the sufficiency. Suppose that the inequalities (20) are true. Let us prove that the martingale measure $\mu_{0}$ is unique. For this purpose, we prove that for every martingale the representation (21) is true with validity of equalities (22), (23).

Let us note that the equality (26) is true if for $d_{i_{1}, \ldots, i_{n-1}}$ to choose (37) since the equalities

$$
\begin{gathered}
{\left[\frac{\int_{A^{i_{1}, \ldots, i_{n-1}, 1}} m\left(\omega_{1}, \ldots, \omega_{N}\right) d \mu_{0}}{\mu_{0}\left(A^{i_{1}, \ldots, i_{n-1}, 1}\right)}-\frac{\int_{A^{i_{1}, \ldots, i_{n-1}}} m\left(\omega_{1}, \ldots, \omega_{N}\right) d \mu_{0}}{\mu_{0}\left(A^{i_{1}, \ldots, i_{n-1}}\right)}\right] \times} \\
{\left[\frac{\int_{A^{i_{1}, \ldots, i_{n-1}, 1}} S_{N}\left(\omega_{1}, \ldots, \omega_{N}\right) d \mu_{0}}{\mu_{0}\left(A^{i_{1}, \ldots, i_{n-1}, 1}\right)}-\frac{A^{i_{1}, \ldots, i_{n-1}}}{\mu_{0}\left(A^{i_{1}, \ldots, i_{n-1}}\right)} S\left(\omega_{1}, \ldots, \omega_{N}\right) d \mu_{0}\right.} \\
=
\end{gathered}
$$




$$
\begin{gathered}
{\left[\frac{\int_{i_{1}, \ldots, i_{n-1}, 2} m\left(\omega_{1}, \ldots, \omega_{N}\right) d \mu_{0}}{\mu_{0}\left(A^{i_{1}, \ldots, i_{n-1}, 2}\right)}-\frac{\int^{i_{1}, \ldots, i_{n-1}} m\left(\omega_{1}, \ldots, \omega_{N}\right) d \mu_{0}}{\mu_{0}\left(A^{i_{1}, \ldots, i_{n-1}}\right)}\right] \times} \\
{\left[\frac{\int_{A^{i_{1}, \ldots, i_{n-1}, 2}} S_{N}\left(\omega_{1}, \ldots, \omega_{N}\right) d \mu_{0}}{\mu_{0}\left(A^{i_{1}, \ldots, i_{n-1}, 2}\right)}-\frac{A_{A_{1}, \ldots, i_{n-1}} S_{N}\left(\omega_{1}, \ldots, \omega_{N}\right) d \mu_{0}}{\mu_{0}\left(A^{i_{1}, \ldots, i_{n-1}}\right)}\right]^{-1}=} \\
d_{i_{1}, \ldots, i_{n-1}}
\end{gathered}
$$

are valid.

Taking into account the equality (26) and the equalities

$$
\begin{aligned}
& d_{i_{1}, \ldots, i_{n-1}} \chi_{A^{i_{1}, \ldots, i_{n-1}}}\left(\omega_{1}, \ldots, \omega_{n-1}\right) \times \\
& {\left[\frac{\chi_{A^{i_{1}, \ldots, i_{n-1}, 1}}\left(\omega_{1}, \ldots, \omega_{n}\right)}{\mu_{0}\left(A^{i_{1}, \ldots, i_{n-1}, 1}\right)} \int_{A^{i_{1}, \ldots, i_{n-1}, 1}} S_{N}\left(\omega_{1}, \ldots, \omega_{N}\right) d \mu_{0}+\right.} \\
& \frac{\chi_{A^{i_{1}, \ldots, i_{n-1}, 2}}\left(\omega_{1}, \ldots, \omega_{n}\right)}{\mu_{0}\left(A^{i_{1}, \ldots, i_{n-1}, 2}\right)} \int_{A^{i_{1}, \ldots, i_{n-1}, 2}} S_{N}\left(\omega_{1}, \ldots, \omega_{N}\right) d \mu_{0}- \\
& \left.\frac{\chi_{A^{i_{1}, \ldots, i_{n-1}}}\left(\omega_{1}, \ldots, \omega_{n}\right)}{\mu_{0}\left(A^{i_{1}, \ldots, i_{n-1}}\right)} \int_{A^{i_{1}, \ldots, i_{n-1}}} S_{N}\left(\omega_{1}, \ldots, \omega_{N}\right) d \mu_{0}\right]= \\
& d_{i_{1}, \ldots, i_{n-1}} \chi_{A^{i_{1}, \ldots, i_{n-1}}}\left(\omega_{1}, \ldots, \omega_{n-1}\right) \times \\
& \sum_{j_{1}=1, \ldots j_{n-1}=1}^{2}\left[\frac{\chi_{A^{j_{1}, \ldots, j_{n-1}, 1}\left(\omega_{1}, \ldots, \omega_{n}\right)}}{\mu_{0}\left(A^{j_{1}, \ldots, j_{n-1}, 1}\right)} \int_{A^{j_{1}, \ldots, j_{n-1}, 1}} S_{N}\left(\omega_{1}, \ldots, \omega_{N}\right) d \mu_{0}+\right.
\end{aligned}
$$



$$
\begin{aligned}
& \left.\frac{\chi_{A^{j_{1}, \ldots, j_{n-1}}}\left(\omega_{1}, \ldots, \omega_{n}\right)}{\mu_{0}\left(A^{j_{1}, \ldots, j_{n-1}}\right)} \int_{A^{j_{1}, \ldots, j_{n-1}}} S_{N}\left(\omega_{1}, \ldots, \omega_{N}\right) d \mu_{0}\right]= \\
& d_{i_{1}, \ldots, i_{n-1}} \chi_{A^{i_{1}, \ldots, i_{n-1}}}\left(\omega_{1}, \ldots, \omega_{n}\right)\left[S_{n}\left(\omega_{1}, \ldots, \omega_{n-1}, \omega_{n}\right)-S_{n-1}\left(\omega_{1}, \ldots, \omega_{n-1}\right)\right],
\end{aligned}
$$

we have

$$
\frac{\chi_{A^{i_{1}, \ldots, i_{n-1}, 1}\left(\omega_{1}, \ldots, \omega_{n}\right)} \int_{0}\left(A^{i_{1}, \ldots, i_{n-1}, 1}\right)}{A^{i_{1}, \ldots, i_{n-1}, 1}} m\left(\omega_{1}, \ldots, \omega_{N}\right) d \mu_{0}+
$$




$$
\begin{gathered}
\frac{\chi_{A^{i_{1}, \ldots, i_{n-1}, 2}}\left(\omega_{1}, \ldots, \omega_{n}\right)}{\mu_{0}\left(A^{i_{1}, \ldots, i_{n-1}, 2}\right)} \int_{A^{i_{1}, \ldots, i_{n-1}, 2}} m\left(\omega_{1}, \ldots, \omega_{N}\right) d \mu_{0}- \\
\frac{\chi_{A^{i_{1}, \ldots, i_{n-1}}}\left(\omega_{1}, \ldots, \omega_{n}\right)}{\mu_{0}\left(A^{i_{1}, \ldots, i_{n-1}}\right)} \int_{A^{i_{1}, \ldots, i_{n-1}}} m\left(\omega_{1}, \ldots, \omega_{N}\right) d \mu_{0}= \\
d_{i_{1}, \ldots, i_{n-1}} \chi_{A^{i_{1}, \ldots, i_{n-1}}}\left(\omega_{1}, \ldots, \omega_{n}\right)\left[S_{n}\left(\omega_{1}, \ldots, \omega_{n-1}, \omega_{n}\right)-S_{n-1}\left(\omega_{1}, \ldots, \omega_{n-1}\right)\right] .
\end{gathered}
$$

Summing over all indexes $i_{1}, \ldots, i_{n-1}$ left and right hand sides of the equality (42) we obtain the equality

$$
\begin{gathered}
m_{n}\left(\omega_{1}, \ldots, \omega_{n}\right)-m_{n-1}\left(\omega_{1}, \ldots, \omega_{n-1}\right)= \\
C_{n}\left(\omega_{1}, \ldots, \omega_{n-1}\right)\left[S_{n}\left(\omega_{1}, \ldots, \omega_{n-1}, \omega_{n}\right)-S_{n-1}\left(\omega_{1}, \ldots, \omega_{n-1}\right)\right] \\
C_{n}\left(\omega_{1}, \ldots, \omega_{n-1}\right)=\sum_{i_{1}=1, \ldots, i_{n-1}=1}^{2} d_{i_{1}, \ldots, i_{n-1}} \chi_{A^{i_{1}, \ldots, i_{n-1}}}\left(\omega_{1}, \ldots, \omega_{n-1}\right) .
\end{gathered}
$$

We proved that for every martingale the representation (21) is true, due to the conditions (20). Let us prove that the martingale measure is unique. Suppose that there are at most two martingale measures $\mu_{0}^{1}$ and $\mu_{0}^{2}$. If to put $m\left(\omega_{1}, \ldots, \omega_{N}\right)=$ $\chi_{A}\left(\omega_{1}, \ldots, \omega_{N}\right)$, then

$$
\begin{gathered}
\chi_{A}\left(\omega_{1}, \ldots, \omega_{N}\right)= \\
\sum_{n=1}^{N} C_{n}\left(\omega_{1}, \ldots, \omega_{n-1}\right)\left[S_{n}\left(\omega_{1}, \ldots, \omega_{n-1}, \omega_{n}\right)-S_{n-1}\left(\omega_{1}, \ldots, \omega_{n-1}\right)\right]+c_{0} .
\end{gathered}
$$

From this representation, we obtain the equalities $\mu_{0}^{1}(A)=\mu_{0}^{2}(A)=c_{0}, A \in \mathcal{F}_{N}$. Contradiction. The last proves Theorem 1.

Next Theorem is concerned the case as the set of martingale measures consists of one measure.

Theorem 2. On the probability space $\left\{\Omega_{N}, \mathcal{F}_{N}, P_{N}\right\}$, being the direct product of the probability spaces $\left\{\Omega_{i}^{0}, \mathcal{F}_{i}^{0}, P_{i}^{0}\right\}$, suppose that the evolution of risky asset is given by the formula (11), then the set of martingale measures, being equivalent to the measure $P_{N}$, consists of one point

$$
\begin{gathered}
\mu_{0}(A)= \\
\sum_{i_{1}=1}^{2} \ldots \sum_{i_{N}=1}^{2} \prod_{n=1}^{N} \psi_{n}\left(\omega_{1}^{i_{1}}, \ldots, \omega_{n}^{i_{n}}\right) \chi_{A}\left(\omega_{1}^{i_{1}}, \ldots, \omega_{N}^{i_{N}}\right), \quad A \in \mathcal{F}_{N} .
\end{gathered}
$$

The fair price of contract with option $\varphi_{0}$ of European type with the payoff function $\varphi\left(\omega_{1}, \ldots, \omega_{N}\right)$ is given by the formula

$$
\varphi_{0}=\sum_{i_{1}=1}^{2} \ldots \sum_{i_{N}=1}^{2} \prod_{n=1}^{N} \psi_{n}\left(\omega_{1}^{i_{1}}, \ldots, \omega_{n}^{i_{n}}\right) \varphi\left(\omega_{1}^{i_{1}}, \ldots, \omega_{N}^{i_{N}}\right),
$$


where

$$
\begin{gathered}
\psi_{n}\left(\omega_{1}, \ldots, \omega_{n}^{-}\right)=\chi_{\Omega_{n}^{-}}\left(\omega_{1}, \ldots, \omega_{n-1}, \omega_{n}\right) \psi_{n}^{1}\left(\omega_{1}, \ldots, \omega_{n}\right)+ \\
\chi_{\Omega_{n}^{+}}\left(\omega_{1}, \ldots, \omega_{n-1}, \omega_{n}\right) \psi_{n}^{2}\left(\omega_{1}, \ldots, \omega_{n}\right) \\
\psi_{n}^{1}\left(\omega_{1}, \ldots, \omega_{n-1}, \omega_{n}\right)= \\
\frac{\Delta S_{n}^{+}\left(\omega_{1}, \ldots, \omega_{n-1}, \omega_{n}^{2}\right)}{V_{n}\left(\omega_{1}, \ldots, \omega_{n-1}, \omega_{n}^{1}, \omega_{n}^{2}\right)}, \quad\left(\omega_{1}, \ldots, \omega_{n-1}\right) \in \Omega_{n-1}, \\
\psi_{n}^{2}\left(\omega_{1}, \ldots, \omega_{n-1}, \omega_{n}\right)=
\end{gathered}
$$

$$
\frac{\Delta S_{n}^{-}\left(\omega_{1}, \ldots, \omega_{n-1}, \omega_{n}^{1}\right)}{V_{n}\left(\omega_{1}, \ldots, \omega_{n-1}, \omega_{n}^{1}, \omega_{n}^{2}\right)}, \quad\left(\omega_{1}, \ldots, \omega_{n-1}\right) \in \Omega_{n-1} .
$$

Proof. Since

$$
\begin{gathered}
\psi_{n}^{1}\left(\omega_{1}, \ldots, \omega_{n-1}, \omega_{n}\right)= \\
\frac{\Delta S_{n}^{+}\left(\omega_{1}, \ldots, \omega_{n-1}, \omega_{n}^{2}\right)}{V_{n}\left(\omega_{1}, \ldots, \omega_{n-1}, \omega_{n}^{1}, \omega_{n}^{2}\right)}>0, \quad\left(\omega_{1}, \ldots, \omega_{n-1}\right) \in \Omega_{n-1}, \\
\psi_{n}^{2}\left(\omega_{1}, \ldots, \omega_{n-1}, \omega_{n}\right)= \\
\frac{\Delta S_{n}^{-}\left(\omega_{1}, \ldots, \omega_{n-1}, \omega_{n}^{1}\right)}{V_{n}\left(\omega_{1}, \ldots, \omega_{n-1}, \omega_{n}^{1}, \omega_{n}^{2}\right)}>0, \quad\left(\omega_{1}, \ldots, \omega_{n-1}\right) \in \Omega_{n-1},
\end{gathered}
$$

we have

$$
\begin{gathered}
\psi_{n}\left(\omega_{1}, \ldots, \omega_{n}\right)=\chi_{\Omega_{n}^{-}}\left(\omega_{1}, \ldots, \omega_{n-1}, \omega_{n}\right) \psi_{n}^{1}\left(\omega_{1}, \ldots, \omega_{n}\right)+ \\
\chi_{\Omega_{n}^{+}}\left(\omega_{1}, \ldots, \omega_{n-1}, \omega_{n}\right) \psi_{n}^{2}\left(\omega_{1}, \ldots, \omega_{n}\right)>0, \quad\left(\omega_{1}, \ldots, \omega_{n}\right) \in \Omega_{n} .
\end{gathered}
$$

From this, it follows that $\mu_{0}(A)>0$ for every $A \in \mathcal{F}_{N}$. It means that $\mu_{0}(A)$ is equivalent to $P_{N}$. The inequality

$$
\begin{gathered}
S_{n}\left(\omega_{1}, \ldots, \omega_{n-1}, \omega_{n}^{1}\right)=\prod_{i=1}^{n-1}\left(1+a_{i}\left(\omega_{1}, \ldots, \omega_{i}\right) \eta_{i}\left(\omega_{i}\right)\right)\left(1+a_{n}\left(\omega_{1}, \ldots, \omega_{n}^{1}\right) \eta_{i}\left(\omega_{n}^{1}\right)\right) \neq \\
S_{n}\left(\omega_{1}, \ldots, \omega_{n-1}, \omega_{n}^{2}\right)= \\
\prod_{i=1}^{n-1}\left(1+a_{i}\left(\omega_{1}, \ldots, \omega_{i}\right) \eta_{i}\left(\omega_{i}\right)\right)\left(1+a_{n}\left(\omega_{1}, \ldots, \omega_{n}^{2}\right) \eta_{i}\left(\omega_{n}^{2}\right)\right), \quad n=\overline{1, N}
\end{gathered}
$$

is true, since

$$
\left(1+a_{n}\left(\omega_{1}, \ldots, \omega_{n}^{1}\right) \eta_{i}\left(\omega_{n}^{1}\right)\right) \neq
$$




$$
\left(1+a_{n}\left(\omega_{1}, \ldots, \omega_{n}^{2}\right) \eta_{i}\left(\omega_{n}^{2}\right)\right), \quad n=\overline{1, N}
$$

due to the suppositions relative to the evolutions of risky asset, given by the formula (11). Thanks to Theorem 1 , the martingale measure $\mu_{0}$ is unique.

To prove the rest statement of Theorem 2, we need to construct the self-financing strategy $\pi$ such that the capital corresponding this strategy on $(B, S)$ market satisfies the condition

$$
X_{N}^{\pi}=\varphi\left(\omega_{1}, \ldots, \omega_{n-1}, \omega_{N}\right) .
$$

Let us consider the martingale

$$
m_{n}\left(\omega_{1}, \ldots, \omega_{n-1}, \omega_{n}\right)=E^{\mu_{0}}\left\{\varphi\left(\omega_{1}, \ldots, \omega_{n-1}, \omega_{N}\right) \mid \mathcal{F}_{n}\right\}
$$

Due to Theorem 1 , for the finite martingale $\left\{m_{n}\left(\omega_{1}, \ldots, \omega_{n-1}, \omega_{n}\right)\right\}_{n=0}^{N}$ relative to the the measure $\mu_{0}(A)$ the representation

$$
\begin{gathered}
m_{n}\left(\omega_{1}, \ldots, \omega_{n-1}, \omega_{n}\right)= \\
\sum_{i=1}^{n} C_{i}\left(\omega_{1}, \ldots, \omega_{i-1}\right)\left[S_{i}\left(\omega_{1}, \ldots, \omega_{i-1}, \omega_{i}\right)-S_{i-1}\left(\omega_{1}, \ldots, \omega_{i-1}\right)\right]+ \\
m_{0}, \quad n=\overline{1, N}
\end{gathered}
$$

is true, where $C_{i}\left(\omega_{1}, \ldots, \omega_{i-1}\right)$ is $\mathcal{F}_{i-1}$ measurable random value, and $m_{0}=$ $E^{\mu_{0}} \varphi\left(\omega_{1}, \ldots, \omega_{n-1}, \omega_{N}\right)$.

If to put $\pi=\left\{\beta_{n}, \gamma_{n}\right\}_{n=0}^{N}$, where

$$
\gamma_{n}=C_{n}\left(\omega_{1}, \ldots, \omega_{n-1}\right), \quad \beta_{n}=m_{n-1}\left(\omega_{1}, \ldots, \omega_{n-1}\right)-\gamma_{n} S_{n-1}\left(\omega_{1}, \ldots, \omega_{n-1}\right),
$$

then it easy to see that $\pi$ is self-financed strategy. Really,

$$
\begin{gathered}
\Delta \beta_{n} B_{n-1}+\gamma_{n} \Delta S_{n-1}=\Delta \beta_{n}+\Delta \gamma_{n} S_{n-1}= \\
m_{n-1}-\gamma_{n} S_{n-1}-m_{n-2}+\gamma_{n-1} S_{n-2}+\left(\gamma_{n}-\gamma_{n-1}\right) S_{n-1}= \\
m_{n-1}-m_{n-2}-\gamma_{n-1}\left(S_{n-1}-S_{n-2}\right)=0 .
\end{gathered}
$$

$\mathcal{F}_{n-1}$-measurability of $\left(\beta_{n}, \gamma_{n}\right)$ is evident.

It is easy to show that

$$
X_{n}\left(\omega_{1}, \ldots, \omega_{n}\right)=\beta_{n} B_{n}+\gamma_{n} S_{n}=m_{n}\left(\omega_{1}, \ldots, \omega_{n}\right)
$$

Therefore,

$$
X_{0}=m_{0}=E^{\mu_{0}} \varphi\left(\omega_{1}, \ldots, \omega_{n-1}, \omega_{N}\right), \quad X_{N}=\varphi\left(\omega_{1}, \ldots, \omega_{n-1}, \omega_{N}\right) .
$$




\section{Complete Market Hedging}

In this section, the securities market is constructed, the evolution of which occurs in accordance with Formula (11). Possible for this was the observation that with respect to a certain class of evolutions of risky assets, the family of martingale measures is invariant. This fact turned out to be crucial for the construction of models of non-arbitrage markets. In papers [10], [11], such a possibility of the existence of non-arbitrage markets is established on the basis of the Hahn-Banach Theorem. This beautiful result has the disadvantage that it does not provide an algorithm for constructing models of non-arbitrage markets. How to build them having the evolution of risky assets is practically a difficult problem.

In Proposition 1, we establish the form of measurable transformations relative to which the only measure is invariant. Using that, a model of the securities market is built, which is complete. This result is constructive in contrast to the existence theorem from [10], [11]. Our denotations in this section are the same as in the previous section. We consider the evolution of risky assets given by the formula (11) on the same probability space.

Proposition 1. On the probability space $\left\{\Omega_{N}, \mathcal{F}_{N}, P_{N}\right\}$, being the direct product of the probability spaces $\left\{\Omega_{i}^{0}, \mathcal{F}_{i}^{0}, P_{i}^{0}\right\}$, let the evolution of risky asset be given by the formula (11), with $a_{i}\left(\omega_{1}, \ldots, \omega_{i}\right)=b_{i}\left(\omega_{1}, \ldots, \omega_{i-1}\right) f_{i}\left(\omega_{1}, \ldots, \omega_{i}\right)$, where the random variables $f_{i}\left(\omega_{1}, \ldots, \omega_{i}\right), b_{i}\left(\omega_{1}, \ldots, \omega_{i-1}\right)$, satisfy the inequalities

$$
\begin{gathered}
f_{i}\left(\omega_{1}, \ldots, \omega_{i}\right)>0, \quad b_{i}\left(\omega_{1}, \ldots, \omega_{i-1}\right)>0, \quad \max _{\left\{\omega_{1}, \ldots, \omega_{i-1}\right\} \in \Omega_{i-1}} b_{i}\left(\omega_{1}, \ldots, \omega_{i-1}\right)< \\
\frac{1}{\max _{\left\{\omega_{1}, \ldots, \omega_{i-1}\right\} \in \Omega_{i-1}} f_{i}\left(\omega_{1}, \ldots, \omega_{i-1}, \omega_{i}^{1}\right) \eta_{i}^{-}\left(\omega_{i}^{1}\right)}, \quad i=\overline{1, N}
\end{gathered}
$$

For such an evolution, the unique martingale measure $\mu_{0}$ does not depend on the random variables $b_{i}\left(\omega_{1}, \ldots, \omega_{i-1}\right), i=\overline{1, N}$, and it is given by the formula

$$
\begin{gathered}
\mu_{0}(A)=\mu_{\left\{\omega_{1}^{1}, \omega_{1}^{2}\right\}, \ldots,\left\{\omega_{N}^{1}, \omega_{N}^{2}\right\}}(A)= \\
\sum_{i_{1}=1}^{2} \ldots \sum_{i_{N}=1}^{2} \prod_{n=1}^{N} \psi_{n}\left(\omega_{1}^{i_{1}}, \ldots, \omega_{n}^{i_{n}}\right) \chi_{A}\left(\omega_{1}^{i_{1}}, \ldots, \omega_{N}^{i_{N}}\right), \quad A \in \mathcal{F}_{N},
\end{gathered}
$$

where

$$
\begin{gathered}
\psi_{n}\left(\omega_{1}, \ldots, \omega_{n}\right)=\chi_{\Omega_{n}^{-}}\left(\omega_{1}, \ldots, \omega_{n-1}, \omega_{n}\right) \psi_{n}^{1}\left(\omega_{1}, \ldots, \omega_{n}\right)+ \\
\chi_{\Omega_{n}^{+}}\left(\omega_{1}, \ldots, \omega_{n-1}, \omega_{n}\right) \psi_{n}^{2}\left(\omega_{1}, \ldots, \omega_{n}\right), \\
\psi_{n}^{1}\left(\omega_{1}, \ldots, \omega_{n-1}, \omega_{n}\right)=\frac{\Delta S_{n}^{+}\left(\omega_{1}, \ldots, \omega_{n-1}, \omega_{n}^{2}\right)}{V_{n}\left(\omega_{1}, \ldots, \omega_{n-1}, \omega_{n}^{1}, \omega_{n}^{2}\right)}= \\
\frac{f_{n}\left(\omega_{1}, \ldots, \omega_{n-1}, \omega_{n}^{2}\right) \eta_{n}^{+}\left(\omega_{n}^{2}\right)}{f_{n}\left(\omega_{1}, \ldots, \omega_{n-1}, \omega_{n}^{2}\right) \eta_{n}^{+}\left(\omega_{n}^{2}\right)+f_{n}\left(\omega_{1}, \ldots, \omega_{n-1}, \omega_{n}^{1}\right) \eta_{n}^{-}\left(\omega_{n}^{1}\right)} \\
\psi_{n}^{2}\left(\omega_{1}, \ldots, \omega_{n-1}, \omega_{n}\right)=\frac{\Delta S_{n}^{-}\left(\omega_{1}, \ldots, \omega_{n-1}, \omega_{n}^{1}\right)}{V_{n}\left(\omega_{1}, \ldots, \omega_{n-1}, \omega_{n}^{1}, \omega_{n}^{2}\right)}=
\end{gathered}
$$




$$
\frac{f_{n}\left(\omega_{1}, \ldots, \omega_{n-1}, \omega_{n}^{1}\right) \eta_{n}^{-}\left(\omega_{n}^{1}\right)}{f_{n}\left(\omega_{1}, \ldots, \omega_{n-1}, \omega_{n}^{2}\right) \eta_{n}^{+}\left(\omega_{n}^{2}\right)+f_{n}\left(\omega_{1}, \ldots, \omega_{n-1}, \omega_{n}^{1}\right) \eta_{n}^{-}\left(\omega_{n}^{1}\right)} .
$$

Proof. Due to the representation (46) for the measure $\mu_{0}$, to prove Proposition 1 it needs to prove that all $\psi_{n}\left(\omega_{1}, \ldots, \omega_{n}\right), n=\overline{1, N}$, do not depend on the random variables $b_{i}\left(\omega_{1}, \ldots, \omega_{i_{-} 1}\right), i=\overline{1, N}$, where

$$
\begin{gathered}
\psi_{n}\left(\omega_{1}, \ldots, \omega_{n}\right)=\chi_{\Omega_{n}^{-}}\left(\omega_{1}, \ldots, \omega_{n-1}, \omega_{n}\right) \psi_{n}^{1}\left(\omega_{1}, \ldots, \omega_{n}\right)+ \\
\chi_{\Omega_{n}^{+}}\left(\omega_{1}, \ldots, \omega_{n-1}, \omega_{n}\right) \psi_{n}^{2}\left(\omega_{1}, \ldots, \omega_{n}\right), \\
\psi_{n}^{1}\left(\omega_{1}, \ldots, \omega_{n-1}, \omega_{n}\right)= \\
\frac{\Delta S_{n}^{+}\left(\omega_{1}, \ldots, \omega_{n-1}, \omega_{n}^{2}\right)}{V_{n}\left(\omega_{1}, \ldots, \omega_{n-1}, \omega_{n}^{1}, \omega_{n}^{2}\right)}, \quad\left(\omega_{1}, \ldots, \omega_{n-1}\right) \in \Omega_{n-1}, \\
\psi_{n}^{2}\left(\omega_{1}, \ldots, \omega_{n-1}, \omega_{n}\right)= \\
\frac{\Delta S_{n}^{-}\left(\omega_{1}, \ldots, \omega_{n-1}, \omega_{n}^{1}\right)}{V_{n}\left(\omega_{1}, \ldots, \omega_{n-1}, \omega_{n}^{1}, \omega_{n}^{2}\right)}, \quad\left(\omega_{1}, \ldots, \omega_{n-1}\right) \in \Omega_{n-1} .
\end{gathered}
$$

But,

$$
\begin{aligned}
& \Delta S_{n}^{+}\left(\omega_{1}, \ldots, \omega_{n-1}, \omega_{n}^{2}\right)= \\
& S_{n-1}\left(\omega_{1}, \ldots, \omega_{n-1}\right) b_{n}\left(\omega_{1}, \ldots, \omega_{n-1}\right) f_{n}\left(\omega_{1}, \ldots, \omega_{n}^{2}\right) \eta_{n}^{+}\left(\omega_{n}^{2}\right), \\
& \Delta S_{n}^{-}\left(\omega_{1}, \ldots, \omega_{n-1}, \omega_{n}^{1}\right)= \\
& S_{n-1}\left(\omega_{1}, \ldots, \omega_{n-1}\right) b_{n}\left(\omega_{1}, \ldots, \omega_{n-1}\right) f_{n}\left(\omega_{1}, \ldots, \omega_{n}^{1}\right) \eta_{n}^{-}\left(\omega_{n}^{1}\right) .
\end{aligned}
$$

Therefore,

$$
\begin{aligned}
& \frac{\Delta S_{n}^{+}\left(\omega_{1}, \ldots, \omega_{n-1}, \omega_{n}^{2}\right)}{V_{n}\left(\omega_{1}, \ldots, \omega_{n-1}, \omega_{n}^{1}, \omega_{n}^{2}\right)}= \\
& \frac{f_{n}\left(\omega_{1}, \ldots, \omega_{n-1}, \omega_{n}^{2}\right) \eta_{n}^{+}\left(\omega_{n}^{2}\right)}{f_{n}\left(\omega_{1}, \ldots, \omega_{n-1}, \omega_{n}^{2}\right) \eta_{n}^{+}\left(\omega_{n}^{2}\right)+f_{n}\left(\omega_{1}, \ldots, \omega_{n-1}, \omega_{n}^{1}\right) \eta_{n}^{-}\left(\omega_{n}^{1}\right)}, \\
& \frac{\Delta S_{n}^{-}\left(\omega_{1}, \ldots, \omega_{n-1}, \omega_{n}^{1}\right)}{V_{n}\left(\omega_{1}, \ldots, \omega_{n-1}, \omega_{n}^{1}, \omega_{n}^{2}\right)}= \\
& \frac{f_{n}\left(\omega_{1}, \ldots, \omega_{n-1}, \omega_{n}^{1}\right) \eta_{n}^{-}\left(\omega_{n}^{1}\right)}{f_{n}\left(\omega_{1}, \ldots, \omega_{n-1}, \omega_{n}^{2}\right) \eta_{n}^{+}\left(\omega_{n}^{2}\right)+f_{n}\left(\omega_{1}, \ldots, \omega_{n-1}, \omega_{n}^{1}\right) \eta_{n}^{-}\left(\omega_{n}^{1}\right)}, \\
& \left(\omega_{1}, \ldots, \omega_{n-1}\right) \in \Omega_{n-1} .
\end{aligned}
$$

The equalities (67), (68) prove Proposition 1. 
Suppose that the market consists of $d$ assets the evolutions of which are given by the law

$$
S_{n}\left(\left(\omega_{1}, \ldots, \omega_{n}\right)=\left\{S _ { n } ^ { 1 } \left(\left(\omega_{1}, \ldots, \omega_{n}\right), \ldots, S_{n}^{d}\left(\left(\omega_{1}, \ldots, \omega_{n}\right)\right\}, \quad n=\overline{1, N},\right.\right.\right.
$$

where

$$
S_{n}^{k}\left(\left(\omega_{1}, \ldots, \omega_{n}\right)=S_{0}^{k} \prod_{i=1}^{n}\left(1+b_{i}^{k}\left(\omega_{1}, \ldots, \omega_{i-1}\right) f_{i}\left(\omega_{1}, \ldots, \omega_{i}\right) \eta_{i}\left(\omega_{i}\right)\right), \quad k=\overline{1, d}\right.
$$

and the random values $\eta_{i}\left(\omega_{i}\right), f_{i}\left(\omega_{1}, \ldots, \omega_{i}\right), i=\overline{1, N}$, do not depend on $k$, and satisfy inequalities

$$
\begin{gathered}
f_{i}\left(\omega_{1}, \ldots, \omega_{i}\right)>0, \quad b_{i}^{k}\left(\omega_{1}, \ldots, \omega_{i-1}\right)>0, \quad \max _{\left\{\omega_{1}, \ldots, \omega_{i-1}\right\} \in \Omega_{i-1}} b_{i}^{k}\left(\omega_{1}, \ldots, \omega_{i-1}\right)< \\
\frac{1}{\max _{\left\{\omega_{1}, \ldots, \omega_{i-1}\right\} \in \Omega_{i-1}} f_{i}\left(\omega_{1}, \ldots, \omega_{i-1}, \omega_{i}^{1}\right) \eta_{i}^{-}\left(\omega_{i}^{1}\right)}, \quad k=\overline{1, d}, \quad i=\overline{1, N} .
\end{gathered}
$$

Proposition 2. On the probability space $\left\{\Omega_{N}, \mathcal{F}_{N}, P_{N}\right\}$, being the direct product of the probability spaces $\left\{\Omega_{i}^{0}, \mathcal{F}_{i}^{0}, P_{i}^{0}\right\}$, if the evolution of d risky assets is given by the formula (69), (70), then such a market is complete non arbitrage one. The unique martingale measure does not depend on the random variables $b_{i}^{k}\left(\omega_{1}, \ldots, \omega_{i-1}\right), k=$ $\overline{1, d}, \quad i=\overline{1, N}$, and it is determined by the formula (58). For the contingent claims $\varphi_{i}\left(\omega_{1}, \ldots, \omega_{N}\right), i=\overline{1, d}$, the fair prices $\varphi_{0}^{i}$ are given by the formulas

$$
\varphi_{0}^{i}=\sum_{i_{1}=1}^{2} \ldots \sum_{i_{N}=1}^{2} \prod_{n=1}^{N} \psi_{n}\left(\omega_{1}^{i_{1}}, \ldots, \omega_{n}^{i_{n}}\right) \varphi_{i}\left(\omega_{1}^{i_{1}}, \ldots, \omega_{N}^{i_{N}}\right), \quad i=\overline{1, d}
$$

Corollary 1. (Cox, Ross, Rubinstein, see [3]) On the probability space $\left\{\Omega_{N}, \mathcal{F}_{N}, P_{N}\right\}$, being the direct product of the probability spaces $\left\{\Omega_{i}^{0}, \mathcal{F}_{i}^{0}, P_{i}^{0}\right\}$, let the evolution of risky asset is given by the formula

$$
S_{n}^{1}\left(\left(\omega_{1}, \ldots, \omega_{n}\right)=S_{0} \prod_{i=1}^{n}\left(1+\rho_{i}\left(\omega_{i}\right)\right), \quad n=\overline{1, N}\right.
$$

where the random values $\rho_{i}\left(\omega_{i}\right), i=\overline{1, N}$, are such that $\rho_{i}\left(\omega_{i}^{1}\right)=a, \rho_{i}\left(\omega_{i}^{2}\right)=b$, and let the bank account evolution be given by the formula

$$
B_{n}=B_{0}(1+r)^{n}, \quad r>0, \quad B_{0}>0 \quad n=\overline{1, N} .
$$

Then, for the discount evolution of risky asset

$$
S_{n}\left(\left(\omega_{1}, \ldots, \omega_{n}\right)=\frac{S_{0} \prod_{i=1}^{n}\left(1+\rho_{i}\left(\omega_{i}\right)\right)}{B_{0}(1+r)^{n}}, \quad n=\overline{1, N}\right.
$$

the martingale measure $\mu_{0}$ is unique if $a<r<b$. It is a direct product of measures $\mu_{0}^{i}(A), A \in \mathcal{F}_{i}^{0}, i=\overline{1, N}$, given on the measurable space $\left\{\Omega_{i}^{0}, \mathcal{F}_{i}^{0}\right\}$, where $\mu_{0}^{i}\left(\omega_{i}^{1}\right)=$ 
$\frac{b-r}{b-a}, \mu_{0}^{i}\left(\omega_{i}^{2}\right)=\frac{r-a}{b-a}$. The fair price $\varphi_{0}$ of the contingent liability $\varphi_{N}\left(\omega_{1}, \ldots, \omega_{N}\right)$ is given by the formula

$$
\begin{aligned}
& \varphi_{0}=\int_{\Omega_{N}} \varphi_{N}\left(\omega_{1}, \ldots, \omega_{N}\right) d \mu_{0}= \\
& \sum_{i_{1}=1}^{2} \ldots \sum_{i_{N}=1}^{2} \varphi_{N}\left(\omega_{1}^{i_{1}}, \ldots, \omega_{N}^{i_{N}}\right) \prod_{k=1}^{N} \mu_{0}^{k}\left(\omega_{k}^{i_{k}}\right) .
\end{aligned}
$$

Proof. For the discount evolution (75), the representation

$$
S_{n}\left(\left(\omega_{1}, \ldots, \omega_{n}\right)=S_{0} \prod_{i=1}^{n}\left(1+\eta_{i}\left(\omega_{i}\right)\right), \quad n=\overline{1, N}\right.
$$

is true, where $\eta_{i}\left(\omega_{i}\right)=\frac{\left.\rho_{i}\left(\omega_{i}\right)\right)-r}{(1+r)}$. Due to Theorems 1,2 , since $\eta_{i}\left(\omega_{i}^{1}\right)=\frac{a-r}{1+r}<0$, $\eta_{i}\left(\omega_{i}^{2}\right)=\frac{b-r}{1+r}>0$, then the measure $\mu_{0}$ is unique.

Theorem 3. On the probability space $\left\{\Omega_{N}, \mathcal{F}_{N}, P_{N}\right\}$, being the direct product of the probability spaces $\left\{\Omega_{i}^{0}, \mathcal{F}_{i}^{0}, P_{i}^{0}\right\}$, let the evolution of risky asset be given by the formula

$$
S_{n}^{1}\left(\left(\omega_{1}, \ldots, \omega_{n}\right)=S_{0} \prod_{i=1}^{n}\left(1+\rho_{i}\left(\omega_{i}\right)\right), \quad n=\overline{1, N}\right.
$$

where the random values $\rho_{i}\left(\omega_{i}\right), i=\overline{1, N}$, are such that $\rho_{i}\left(\omega_{i}^{1}\right)=b_{i}^{1}, \rho_{i}\left(\omega_{i}^{2}\right)=$ $b_{i}^{2}, i=\overline{1, N}$, and let the bank account evolution be given by the formula

$$
B_{n}=B_{0} \prod_{i=1}^{n}\left(1+r_{i-1}\left(\omega_{i-1}\right)\right), \quad B_{0}>0, \quad n=\overline{1, N}
$$

where the random values $r_{i}\left(\omega_{i}\right), i=\overline{1, N-1}$, are such that $r_{i}\left(\omega_{i}^{1}\right)=r_{i}^{1}, r_{i}\left(\omega_{i}^{2}\right)=$ $r_{i}^{2}, i=\overline{1, N-1}, r_{0}>0$. Then, for the discount evolution of risky asset

$$
S_{n}\left(\left(\omega_{1}, \ldots, \omega_{n}\right)=\frac{S_{0} \prod_{i=1}^{n}\left(1+\rho_{i}\left(\omega_{i}\right)\right)}{B_{0} \prod_{i=1}^{n}\left(1+r_{i-1}\left(\omega_{i-1}\right)\right)}, \quad n=\overline{1, N}\right.
$$

the martingale measure $\mu_{0}$ is unique, if $b_{1}^{1}<r_{0}<b_{1}^{2}, b_{i}^{1}<r_{i-1}^{1}<r_{i-1}^{2}<b_{i}^{2}, i=$ $\overline{2, N}$. It is determined by the formula (58) with

$$
\begin{gathered}
\eta_{1}\left(\omega_{1}\right)=\rho_{1}\left(\omega_{1}\right)-r_{0}, \quad \eta_{i}\left(\omega_{i}\right)=\rho_{i}\left(\omega_{i}\right)-r_{i-1}^{2}, \quad i=\overline{2, N} \\
f_{1}\left(\omega_{1}\right)=\frac{1}{1+r_{0}}, \quad f_{i}\left(\omega_{1}, \ldots, \omega_{i}\right)= \\
\frac{\rho_{i}\left(\omega_{i}\right)-r_{i-1}\left(\omega_{i-1}\right)}{\left(\rho_{i}\left(\omega_{i}\right)-r_{i-1}^{2}\right)\left(1+r_{i-1}\left(\omega_{i-1}\right)\right)}, \quad i=\overline{2, N} .
\end{gathered}
$$


The fair price $\varphi_{0}$ of the contingent liability $\varphi_{N}\left(\omega_{1}, \ldots, \omega_{N}\right)$ is given by the formula

$$
\begin{aligned}
& \varphi_{0}=\int_{\Omega_{N}} \varphi_{N}\left(\omega_{1}, \ldots, \omega_{N}\right) d \mu_{0}= \\
& \sum_{i_{1}=1}^{2} \ldots \sum_{i_{N}=1}^{2} \prod_{n=1}^{N} \psi_{n}\left(\omega_{1}^{i_{1}}, \ldots, \omega_{n}^{i_{n}}\right) \varphi_{N}\left(\omega_{1}^{i_{1}}, \ldots, \omega_{N}^{i_{N}}\right) .
\end{aligned}
$$

Proof. To prove Theorem 3 it is necessary to prove the existence of unique spot measure. The discount evolution (80) can be represented in the form

$$
S_{n}\left(\left(\omega_{1}, \ldots, \omega_{n}\right)=\right.
$$

$$
\frac{S_{0}}{B_{0}} \prod_{i=1}^{n}\left(1+f_{i}\left(\omega_{1}, \ldots, \omega_{i}\right) \eta_{i}\left(\omega_{i}\right)\right), \quad n=\overline{1, N}
$$

where

$$
\begin{gathered}
\eta_{1}\left(\omega_{1}\right)=\rho_{1}\left(\omega_{1}\right)-r_{0}, \quad \eta_{i}\left(\omega_{i}\right)=\rho_{i}\left(\omega_{i}\right)-r_{i-1}^{2}, \quad i=\overline{2, N} \\
f_{1}\left(\omega_{1}\right)=\frac{1}{1+r_{0}}, \quad f_{i}\left(\omega_{1}, \ldots, \omega_{i}\right)= \\
\frac{\rho_{i}\left(\omega_{i}\right)-r_{i-1}\left(\omega_{i-1}\right)}{\left(\rho_{i}\left(\omega_{i}\right)-r_{i-1}^{2}\right)\left(1+r_{i-1}\left(\omega_{i-1}\right)\right)}, \quad i=\overline{2, N}
\end{gathered}
$$

It is evident that $\eta_{i}\left(\omega_{i}^{1}\right)<0, \eta_{i}\left(\omega_{i}^{2}\right)>0, f_{i}\left(\omega_{1}, \ldots, \omega_{i}\right)>0$. Therefore, from the representation (83), (84) it follows that we can construct only one spot measure, which is martingale measure being equivalent to the initial measure $P_{N}$. In accordance with Theorem 1, since $S_{n}\left(\omega_{1}, \ldots, \omega_{n}^{1}\right) \neq S_{n}\left(\omega_{1}, \ldots, \omega_{n}^{2}\right), \quad\left\{\omega_{1}, \ldots, \omega_{n-1}\right\} \in \Omega_{n-1}$ such a measure is unique. Theorem 3 is proved.

Theorem 4. On the probability space $\left\{\Omega_{N}, \mathcal{F}_{N}, P_{N}\right\}$, being the direct product of the probability spaces $\left\{\Omega_{i}^{0}, \mathcal{F}_{i}^{0}, P_{i}^{0}\right\}$, let the evolution of risky asset be given by the formula

$$
S_{n}^{1}\left(\left(\omega_{1}, \ldots, \omega_{n}\right)=S_{0} \prod_{i=1}^{n} e^{\sigma_{i}\left(\omega_{1}, \ldots, \omega_{i-1}\right) \varepsilon_{i}\left(\omega_{i}\right)}, \quad n=\overline{1, N}\right.
$$

where the random values $\varepsilon_{i}\left(\omega_{i}\right), i=\overline{1, N}$, are such that $\varepsilon_{i}\left(\omega_{i}^{1}\right)<0, \varepsilon_{i}\left(\omega_{i}^{2}\right)>0$, $\sigma_{i}\left(\omega_{1}, \ldots, \omega_{i-1}\right) \geq \sigma_{i}^{0}>0, i=\overline{1, N}$, and let the bank account evolution be given by the formula

$$
B_{n}=B_{0} \prod_{i=1}^{n}\left(1+r_{i-1}\left(\omega_{i-1}\right)\right), \quad B_{0}>0, \quad n=\overline{1, N}
$$

where the random values $r_{i}\left(\omega_{i}\right), i=\overline{1, N-1}$, are such that $r_{i}\left(\omega_{i}^{1}\right)=r_{i}^{1}, r_{i}\left(\omega_{i}^{2}\right)=$ $r_{i}^{2}, i=\overline{1, N-1}, r_{0}>0$. Then, for the discount evolution of risky asset 


$$
S_{n}\left(\left(\omega_{1}, \ldots, \omega_{n}\right)=\frac{S_{0} \prod_{i=1}^{n} e^{\sigma_{i}\left(\omega_{1}, \ldots, \omega_{i-1}\right) \varepsilon_{i}\left(\omega_{i}\right)}}{B_{0} \prod_{i=1}^{n}\left(1+r_{i-1}\left(\omega_{i-1}\right)\right)}, \quad n=\overline{1, N}\right.
$$

the martingale measure $\mu_{0}$ is unique, if

$$
\begin{gathered}
\exp \left\{\sigma_{1}^{0} \varepsilon_{1}\left(\omega_{1}^{1}\right)\right\}<r_{0}<\exp \left\{\sigma_{1}^{0} \varepsilon_{1}\left(\omega_{1}^{2}\right)\right\} \\
\exp \left\{\sigma_{i}^{0} \varepsilon_{i}\left(\omega_{i}^{1}\right)\right\}<r_{i-1}^{1}<r_{i-1}^{2}<\exp \left\{\sigma_{i}^{0} \varepsilon_{i}\left(\omega_{i}^{2}\right)\right\}, i=\overline{2, N}
\end{gathered}
$$

It is determined by the formula (58) with

$$
\begin{gathered}
\eta_{1}\left(\omega_{1}\right)=\exp \left\{\sigma_{1}^{0} \varepsilon_{1}\left(\omega_{1}\right)\right\}-r_{0}, \quad f_{1}\left(\omega_{1}\right)=\frac{1}{1+r_{0}}, \\
\eta_{i}\left(\omega_{i}\right)=\exp \left\{\sigma_{i}^{0} \varepsilon_{i}\left(\omega_{i}\right)\right\}-r_{i-1}^{2}, \quad f_{i}\left(\omega_{1}, \ldots, \omega_{i}\right)= \\
\frac{e^{\sigma_{i}\left(\omega_{1}, \ldots, \omega_{i-1}\right) \varepsilon_{i}\left(\omega_{i}\right)}-r_{i-1}\left(\omega_{i-1}\right)}{\left(\exp \left\{\sigma_{i}^{0} \varepsilon_{i}\left(\omega_{i}\right)\right\}-r_{i-1}^{2}\right)\left(1+r_{i-1}\left(\omega_{i-1}\right)\right)}, \quad\left\{\omega_{1}, \ldots, \omega_{i}\right\} \in \Omega_{n}, \quad i=\overline{2, N} .
\end{gathered}
$$

The fair price $\varphi_{0}$ of the contingent liability $\varphi_{N}\left(\omega_{1}, \ldots, \omega_{N}\right)$ is given by the formula

$$
\begin{gathered}
\varphi_{0}=\int_{\Omega_{N}} \varphi_{N}\left(\omega_{1}, \ldots, \omega_{N}\right) d \mu_{0}= \\
\sum_{i_{1}=1}^{2} \ldots \sum_{i_{N}=1}^{2} \prod_{n=1}^{N} \psi_{n}\left(\omega_{1}^{i_{1}}, \ldots, \omega_{n}^{i_{n}}\right) \varphi_{N}\left(\omega_{1}^{i_{1}}, \ldots, \omega_{N}^{i_{N}}\right) .
\end{gathered}
$$

Proof. For the discount evolution (87), the following representation

$$
\begin{gathered}
S_{n}\left(\left(\omega_{1}, \ldots, \omega_{n}\right)=\right. \\
\frac{S_{0}}{B_{0}} \prod_{i=1}^{n}\left(1+f_{i}\left(\omega_{1}, \ldots, \omega_{i}\right) \eta_{i}\left(\omega_{i}\right)\right), \quad n=\overline{1, N}
\end{gathered}
$$

is true, where

$$
\begin{gathered}
\eta_{1}\left(\omega_{1}\right)=\exp \left\{\sigma_{1}^{0} \varepsilon_{1}\left(\omega_{1}\right)\right\}-r_{0}, \quad f_{1}\left(\omega_{1}\right)=\frac{1}{1+r_{0}}, \\
\eta_{i}\left(\omega_{i}\right)=\exp \left\{\sigma_{i}^{0} \varepsilon_{i}\left(\omega_{i}\right)\right\}-r_{i-1}^{2}, \quad f_{i}\left(\omega_{1}, \ldots, \omega_{i}\right)= \\
\frac{e^{\sigma_{i}\left(\omega_{1}, \ldots, \omega_{i-1}\right) \varepsilon_{i}\left(\omega_{i}\right)}-r_{i-1}\left(\omega_{i-1}\right)}{\left(\exp \left\{\sigma_{i}^{0} \varepsilon_{i}\left(\omega_{i}\right)\right\}-r_{i-1}^{2}\right)\left(1+r_{i-1}\left(\omega_{i-1}\right)\right)}, \quad\left\{\omega_{1}, \ldots, \omega_{i}\right\} \in \Omega_{n}, \quad i=\overline{2, N} .
\end{gathered}
$$

It is evident that $\eta_{i}\left(\omega_{i}^{1}\right)<0, \eta_{i}\left(\omega_{i}^{2}\right)>0, f_{i}\left(\omega_{1}, \ldots, \omega_{i}\right)>0$. From this, we obtain that the spot measure exists and it is unique. Theorem 4 is proved. 
On the probability space $\left\{\Omega_{N}, \mathcal{F}_{N}, P_{N}\right\}$, being the direct product of probability spaces $\left\{\Omega_{i}^{0}, \mathcal{F}_{i}^{0}, P_{i}^{0}\right\}$, suppose that the market consists of $d$ assets the evolution of which is given by the law

$$
S_{n}\left(\left(\omega_{1}, \ldots, \omega_{n}\right)=\left\{S _ { n } ^ { 1 } \left(\left(\omega_{1}, \ldots, \omega_{n}\right), \ldots, S_{n}^{d}\left(\left(\omega_{1}, \ldots, \omega_{n}\right)\right\}, \quad n=\overline{1, N},\right.\right.\right.
$$

where

$$
S_{n}^{k}\left(\left(\omega_{1}, \ldots, \omega_{n}\right)=S_{0}^{k} \prod_{i=1}^{n}\left(1+a_{i}^{k} f_{i}\left(\omega_{1}, \ldots, \omega_{i}\right) \eta_{i}\left(\omega_{i}\right)\right), \quad k=\overline{1, d}\right.
$$

and the random values $\eta_{i}\left(\omega_{i}\right), f_{i}\left(\omega_{1}, \ldots, \omega_{i}\right), i=\overline{1, N}$, and constants $a_{i}^{k}$ satisfy the inequalities

$$
\begin{gathered}
\eta_{i}\left(\omega_{i}^{1}\right)<0, \quad \eta_{i}\left(\omega_{i}^{2}\right)>0, \quad f_{i}\left(\omega_{1}, \ldots, \omega_{i}\right)>0, \\
0<a_{i}^{k}<\frac{1}{\max _{\left\{\omega_{1}, \ldots, \omega_{i-1}\right\} \in \Omega_{i-1}} f_{i}\left(\omega_{1}, \ldots, \omega_{i}^{1}\right) \eta_{i}^{-}\left(\omega_{i}^{1}\right)}, \quad i=\overline{1, N}, \quad k=\overline{1, d} .
\end{gathered}
$$

Proposition 3. On the probability space $\left\{\Omega_{N}, \mathcal{F}_{N}, P_{N}\right\}$, being the direct product of the probability spaces $\left\{\Omega_{i}^{0}, \mathcal{F}_{i}^{0}, P_{i}^{0}\right\}$, let the evolution of risky assets be given by the formulas (93), (94), where constants $a_{i}^{k} i=\overline{1, N}, k=\overline{1, d}$, satisfy the inequalities (95). For such an evolution of risky asset the martingale measure $\mu_{0}$ does not depend on $a_{i}^{k}$ and is unique. It is determined by the formula (58). For the contingent claims $\varphi_{N}^{i}\left(\omega_{1}, \ldots, \omega_{N}\right), i=\overline{1, d}$, the fair prices $\varphi_{0}^{i}$ are given by the formulas

$$
\varphi_{0}^{i}=\sum_{i_{1}=1}^{2} \ldots \sum_{i_{N}=1}^{2} \prod_{n=1}^{N} \psi_{n}\left(\omega_{1}^{i_{1}}, \ldots, \omega_{n}^{i_{n}}\right) \varphi_{N}^{i}\left(\omega_{1}^{i_{1}}, \ldots, \omega_{N}^{i_{N}}\right), \quad i=\overline{1, d} .
$$

If $f_{i}\left(\omega_{1}, \ldots, \omega_{i}\right)=1, i=\overline{1, N}$, the unique martingale measure is a direct product of measures $\mu_{0}^{i}(A), A \in \mathcal{F}_{i}^{0}$, given on the measurable space $\left\{\Omega_{i}^{0}, \mathcal{F}_{i}^{0}\right\}, i=\overline{1, N}$, where

$$
\mu_{0}^{i}\left(\omega_{i}^{1}\right)=\frac{\eta_{i}^{+}\left(\omega_{i}^{2}\right)}{\left(\eta_{i}^{-}\left(\omega_{i}^{1}\right)+\eta_{i}^{+}\left(\omega_{i}^{2}\right)\right)}, \quad \mu_{0}^{i}\left(\omega_{i}^{2}\right)=\frac{\eta_{i}^{-}\left(\omega_{i}^{1}\right)}{\left(\eta_{i}^{-}\left(\omega_{i}^{1}\right)+\eta_{i}^{+}\left(\omega_{i}^{2}\right)\right)} .
$$

The fair prices $\varphi_{0}^{i}, i=\overline{1, N}$, of the contingent liabilities $\varphi_{N}^{i}\left(\omega_{1}, \ldots, \omega_{N}\right), i=\overline{1, N}$, are given by the formula

$$
\begin{gathered}
\varphi_{0}^{i}=\int_{\Omega_{N}} \varphi_{N}^{i}\left(\omega_{1}, \ldots, \omega_{N}\right) d \mu_{0}= \\
\sum_{i_{1}=1}^{2} \ldots \sum_{i_{N}=1}^{2} \varphi_{N}^{i}\left(\omega_{1}^{i_{1}}, \ldots, \omega_{N}^{i_{N}}\right) \prod_{k=1}^{N} \mu_{0}^{k}\left(\omega_{k}^{i_{k}}\right) .
\end{gathered}
$$

Suppose that $\left\{g_{k}^{i}\left(X_{N}\right)\right\}_{k=1}^{N}, i=\overline{1, d}$, are the mappings from the set $[0,1]^{N}$ into itself, where $X_{N}=\left\{x_{1}, \ldots, x_{N}\right\}, 0 \leq x_{k} \leq 1, k=\overline{1, N}$. If $S_{0}^{i}, S_{1}^{i}, \ldots, S_{N}^{i}, i=$ $\overline{1, d}$, are the samples of the processes (93), (94) let us denote the order statistics $S_{(0)}^{i}, S_{(1)}^{i}, \ldots, S_{(N)}^{i}, i=\overline{1, d}$, of this samples. Introduce also the denotation

$$
g_{k}^{i}\left(\left[S^{i}\right]_{N}\right)=g_{k}^{i}\left(\frac{S_{(0)}^{i}}{S_{(N)}^{i}}, \ldots, \frac{S_{(N-1)}^{i}}{S_{(N)}^{i}}\right), k=\overline{1, N}, i=\overline{1, d} .
$$


Proposition 4. Suppose that $S_{0}^{i}, S_{1}^{i}, \ldots, S_{N}^{i}$ is a sample of the random processes (93), (94). Then, for the parameters $a_{1}^{i}, \ldots, a_{N}^{i}$ the estimation

$$
\begin{gathered}
a_{1}^{i}=\frac{\left[1-\tau_{0}^{i} \frac{S_{(0)}^{i}}{S_{0}^{i}} g_{1}^{i}\left(\left[S^{i}\right]_{N}\right)\right]}{f_{1} \eta_{1}^{-}\left(\omega_{1}^{1}\right)}, \quad 0<\tau_{0}^{i} \leq 1, \quad i=\overline{1, d}, \\
a_{k}^{i}=\frac{\left[1-\frac{g_{k}^{i}\left(\left[S^{i}\right]_{N}\right)}{g_{k-1}^{i}\left(\left[S^{i}\right]_{N}\right)}\right]}{f_{k} \eta_{k}^{-}\left(\omega_{k}^{1}\right)}, \quad k=\overline{2, N}, \quad i=\overline{1, d},
\end{gathered}
$$

is valid, if for $g_{N}^{i}\left(\left[S^{i}\right]_{N}\right)>0,\left[S^{i}\right]_{N} \in[0,1]^{N}$, the inequalities $g_{1}^{i}\left(\left[S^{i}\right]_{N}\right) \geq$ $g_{2}^{i}\left(\left[S^{i}\right]_{N}\right) \geq \ldots \geq g_{N}^{i}\left(\left[S^{i}\right]_{N}\right)$ are true. If $\tau_{0}^{i}=0$, then $a_{k}^{i}=1, k=\overline{1, N}, i=\overline{1, d}$.

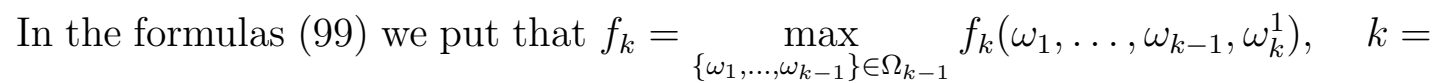
$\overline{1, N}$.

\section{Martingale Measures on Discrete Probability Space}

This section presents all the necessary results for constructing a non-arbitrage incomplete market on a discrete probability space. The conditions under which the entire family of martingale measures is described for the considered class of evolution of risky assets are minimal. In particular, conditions are presented under which the family of martingale measures considered is equivalent to the original measure. They are minimal. The entire set of equivalent martingale measures is a convex combination of a finite number of spot martingale measures. On this basis, new formulas were found for the fair price of the super hedge.

In this section, we put that $\Omega_{i}^{0}=\left\{\omega_{i}^{1}, \ldots, \omega_{i}^{M}\right\}, i=\overline{1, N}$, and we assume that $2<M<\infty$, the $\sigma$-algebra $\mathcal{F}_{i}^{0}$ consists from all subsets of $\Omega_{i}^{0}$. We suppose that $P_{i}^{0}\left(\omega_{i}^{k}\right)>0, \omega_{i}^{k} \in \Omega_{i}^{0}, k=\overline{1, M}$. As before, the probability space $\left\{\Omega_{N}, \mathcal{F}_{N}, P_{N}\right\}$ is a direct product of probability spaces $\left\{\Omega_{i}^{0}, \mathcal{F}_{i}^{0}, P_{i}^{0}\right\}, i=\overline{1, N}$. Sometimes, any elementary event $\omega_{i}^{k} \in \Omega_{i}^{0}$ it is convenient to denote by $\omega_{i}$ not indicating the index $k$. Further, we use the both denotations. As in section 2, we introduce filtration $\mathcal{F}_{n}$ on the probability space $\left\{\Omega_{N}, \mathcal{F}_{N}, P_{N}\right\}$. As before, it is convenient to introduce the family of probability spaces $\left\{\Omega_{n}, \mathcal{F}_{n}, P_{n}\right\}, n=\overline{1, N}$, being a direct product of probability spaces $\left\{\Omega_{i}^{0}, \mathcal{F}_{i}^{0}, P_{i}^{0}\right\}, i=\overline{1, n}$.

The evolution of risky assets is given by the formula (8) with the assumptions given in the section 2 . In this case

$$
\Omega_{n}^{-}=\Omega_{n-1} \times \Omega_{n}^{0-}, \Omega_{n}^{+}=\Omega_{n-1} \times \Omega_{n}^{0+},
$$

where $\Omega_{n}^{0-}=\left\{\omega_{n} \in \Omega_{n}^{0}, \eta_{n}\left(\omega_{n}\right) \leq 0\right\}, \Omega_{n}^{0+}=\left\{\omega_{n} \in \Omega_{n}^{0}, \eta_{n}\left(\omega_{n}\right)>0\right\}$, $P_{n}^{0}\left(\left\{\omega_{n}, \eta_{n}\left(\omega_{n}\right)>0\right\}\right)>0, P_{n}^{0}\left(\left\{\omega_{n}, \eta_{n}\left(\omega_{n}\right)<0\right\}\right)>0$. Further, we also use the measurable space with measure

$$
\left\{\prod_{i=1}^{N}\left[\Omega_{i}^{0-} \times \Omega_{i}^{0+}\right], \prod_{i=1}^{N}\left[\mathcal{F}_{i}^{0-} \times \mathcal{F}_{i}^{0+}\right], \prod_{i=1}^{N}\left[P_{i}^{0-} \times P_{i}^{0+}\right]\right\} .
$$


The measure $P_{n}^{0-}$ is a contraction of the measure $P_{n}^{0}$ on the $\sigma$-algebra $\mathcal{F}_{n}^{0-}=$ $\Omega_{n}^{0-} \cap \mathcal{F}_{n}^{0}, P_{n}^{0+}$ is a contraction of the measure $P_{n}^{0}$ on the $\sigma$-algebra $\mathcal{F}_{n}^{0+}=\Omega_{n}^{0+} \cap \mathcal{F}_{n}^{0}$. Additionally, we assume

$$
P_{n}^{0}\left(\left\{\omega_{n} \in \Omega_{n}^{0},\left|\eta_{n}\left(\omega_{n}\right)\right|<\infty\right\}\right)=1
$$

In this case, Lemma 1 (see [1]) is formulated as follows

Lemma 2. Suppose that for $\Omega_{n}^{a}, a=-,+, n=\overline{1, N}$, the representations (100) are true. If the conditions

$$
\begin{gathered}
B_{n, i}^{0-} \cap B_{n, j}^{0-}=\emptyset, \quad i \neq j, \\
B_{n, s}^{0+} \cap B_{n, l}^{0+}=\emptyset, s \neq l, \quad k=\overline{1, N_{n}}, \\
\Omega_{n}^{0-}=\bigcup_{i=1}^{N_{n}} B_{n, i}^{0-}, \quad \Omega_{n}^{0+}=\bigcup_{i=1}^{N_{n}} B_{n, i}^{0+}, \\
P_{n}^{0}\left(\Omega_{n}^{0-} \backslash B_{n, i}^{0,-}\right)>0, \quad i=\overline{1, I_{n}}, \quad I_{n}>1, \quad n=\overline{1, N}, \\
P_{n}^{0}\left(\Omega_{n}^{0+} \backslash B_{n, s}^{0,+}\right)>0, \quad s=\overline{1, S_{n}}, \quad S_{n}>1, \quad n=\overline{1, N}, \quad \overline{1, N}, \\
P_{n}^{0}\left(B_{n, i}^{0,-}\right)>0, \quad i=\overline{1, I_{n}}, \quad I_{n}>1, \quad n=\overline{1, N}, \\
P_{n}^{0}\left(B_{n, s}^{0,+}\right)>0, \quad s=\overline{1, S_{n}}, \quad S_{n}>1, \quad n=\overline{1, N}, \quad n=\overline{1, N}, \\
\int_{\Omega_{N}}^{\Delta S_{n}^{-}\left(\omega_{1}, \ldots, \omega_{n-1}, \omega_{n}\right) d P_{N}<\infty,} \quad
\end{gathered}
$$

are true, then the set of bounded strictly positive random values $\alpha_{n}\left(\{\omega\}_{n}^{1} ;\{\omega\}_{n}^{2}\right)$, satisfying the conditions (14) - (16), (see [1]) is a nonempty set.

Lemma 3. Suppose that the conditions of Lemma 2 are true. For the measure $\mu_{0}(A), A \in \mathcal{F}_{N}$, constructed by the recurrent relations (23) - (25),(see [1]) the representation

$$
\mu_{0}(A)=\int_{\Omega_{N}} \prod_{n=1}^{N} \psi_{n}\left(\omega_{1}, \ldots, \omega_{n}\right) \chi_{A}\left(\omega_{1}, \ldots, \omega_{N}\right) \prod_{i=1}^{N} d P_{i}^{0}\left(\omega_{i}\right)
$$

is true and $\mu_{0}\left(\Omega_{N}\right)=1$, that is, the measure $\mu_{0}(A)$ is a probability measure being equivalent to the measure $P_{N}$, where we put

$$
\begin{gathered}
\psi_{n}\left(\omega_{1}, \ldots, \omega_{n}\right)=\chi_{\Omega_{n}^{0-}}\left(\omega_{n}\right) \psi_{n}^{1}\left(\omega_{1}, \ldots, \omega_{n}\right)+ \\
\chi_{\Omega_{n}^{0+}}\left(\omega_{n}\right) \psi_{n}^{2}\left(\omega_{1}, \ldots, \omega_{n}\right) \\
\psi_{n}^{1}\left(\omega_{1}, \ldots, \omega_{n-1}, \omega_{n}\right)= \\
\int_{\Omega_{n}^{0}} \chi_{\Omega_{n}^{0+}}\left(\omega_{n}^{2}\right) \alpha_{n}\left(\left\{\omega_{1}, \ldots, \omega_{n-1}, \omega_{n}\right\} ;\left\{\omega_{1}, \ldots, \omega_{n-1}, \omega_{n}^{2}\right\}\right) \times
\end{gathered}
$$




$$
\begin{aligned}
& \frac{\Delta S_{n}^{+}\left(\omega_{1}, \ldots, \omega_{n-1}, \omega_{n}^{2}\right)}{V_{n}\left(\omega_{1}, \ldots, \omega_{n-1}, \omega_{n}^{1}, \omega_{n}^{2}\right)} d P_{n}^{0}\left(\omega_{n}^{2}\right), \quad\left(\omega_{1}, \ldots, \omega_{n-1}\right) \in \Omega_{n-1}, \\
& \psi_{n}^{2}\left(\omega_{1}, \ldots, \omega_{n-1}, \omega_{n}\right)= \\
& \quad \int_{\Omega_{n}^{0}} \chi_{\Omega_{n}^{0-}}\left(\omega_{n}^{1}\right) \alpha_{n}\left(\left\{\omega_{1}, \ldots, \omega_{n-1}, \omega_{n}^{1}\right\} ;\left\{\omega_{1}, \ldots, \omega_{n-1}, \omega_{n}\right\}\right) \times \\
& \frac{\Delta S_{n}^{-}\left(\omega_{1}, \ldots, \omega_{n-1}, \omega_{n}^{1}\right)}{V_{n}\left(\omega_{1}, \ldots, \omega_{n-1}, \omega_{n}^{1}, \omega_{n}^{2}\right)} d P_{n}^{0}\left(\omega_{n}^{1}\right), \quad\left(\omega_{1}, \ldots, \omega_{n-1}\right) \in \Omega_{n-1} .
\end{aligned}
$$

Proof. We only need to prove that $\psi_{n}\left(\omega_{1}, \ldots, \omega_{n}\right)>0, n=\overline{1, N}$. Suppose that

$$
\alpha_{n}\left(\left\{\omega_{1}^{1}, \ldots, \omega_{n-1}^{1}, \omega_{n}^{1}\right\} ;\left\{\omega_{1}^{2}, \ldots, \omega_{n-1}^{2}, \omega_{n}^{2}\right\}\right)=\alpha_{n}^{1}\left(\omega_{n}^{1}\right) \alpha_{n}^{2}\left(\omega_{n}^{2}\right)
$$

where

$$
\alpha_{n}^{1}\left(\omega_{n}^{1}\right)>0, \quad \omega_{n}^{1} \in \Omega_{n}^{0-}, \quad \alpha_{n}^{2}\left(\omega_{n}^{2}\right)>0, \quad \omega_{n}^{2} \in \Omega_{n}^{0+}
$$

Since

$$
\begin{aligned}
& \Delta S_{n}^{-}\left(\omega_{1}, \ldots, \omega_{n-1}, \omega_{n}^{1}\right)=S_{n-1}\left(\omega_{1}, \ldots, \omega_{n-1}\right) a_{n}\left(\omega_{1}, \ldots, \omega_{n-1}, \omega_{n}^{1}\right) \eta_{n}^{-}\left(\omega_{n}^{1}\right) \\
& \Delta S_{n}^{+}\left(\omega_{1}, \ldots, \omega_{n-1}, \omega_{n}^{2}\right)=S_{n-1}\left(\omega_{1}, \ldots, \omega_{n-1}\right) a_{n}\left(\omega_{1}, \ldots, \omega_{n-1}, \omega_{n}^{2}\right) \eta_{n}^{+}\left(\omega_{n}^{2}\right)
\end{aligned}
$$

where

$$
\begin{gathered}
\eta_{n}^{-}\left(\omega_{n}\right)=-\chi_{\Omega_{n}^{0-}}\left(\omega_{n}\right) \eta_{n}\left(\omega_{n}\right), \quad \eta_{n}^{+}\left(\omega_{n}\right)=\chi_{\Omega_{n}^{0+}}\left(\omega_{n}\right) \eta_{n}\left(\omega_{n}\right), \\
a_{n}\left(\omega_{1}, \ldots, \omega_{n-1}, \omega_{n}^{1}\right)>0, \quad a_{n}\left(\omega_{1}, \ldots, \omega_{n-1}, \omega_{n}^{2}\right)>0 .
\end{gathered}
$$

Therefore,

$$
\begin{gathered}
\psi_{n}^{1}\left(\omega_{1}, \ldots, \omega_{n-1}, \omega_{n}\right)= \\
\int_{\Omega_{n}^{0}} \chi_{\Omega_{n}^{0+}}\left(\omega_{n}^{2}\right) \alpha_{n}\left(\left\{\omega_{1}, \ldots, \omega_{n-1}, \omega_{n}\right\} ;\left\{\omega_{1}, \ldots, \omega_{n-1}, \omega_{n}^{2}\right\}\right) \times \\
\frac{\Delta S_{n}^{+}\left(\omega_{1}, \ldots, \omega_{n-1}, \omega_{n}^{2}\right)}{V_{n}\left(\omega_{1}, \ldots, \omega_{n-1}, \omega_{n}^{1}, \omega_{n}^{2}\right)} d P_{n}^{0}\left(\omega_{n}^{2}\right)= \\
S_{n-1}\left(\omega_{1}, \ldots, \omega_{n-1}\right) \alpha_{n}^{1}\left(\omega_{n}\right) \int_{\Omega_{n}^{0}} \chi_{\Omega_{n}^{0+}}\left(\omega_{n}^{2}\right) \alpha_{n}^{2}\left(\omega_{n}\right) \times \\
\frac{a_{n}\left(\omega_{1}, \ldots, \omega_{n-1}, \omega_{n}^{2}\right) \eta_{n}^{+}\left(\omega_{n}^{2}\right)}{V_{n}\left(\omega_{1}, \ldots, \omega_{n-1}, \omega_{n}^{1}, \omega_{n}^{2}\right)} d P_{n}^{0}\left(\omega_{n}^{2}\right)>0, \quad\left(\omega_{1}, \ldots, \omega_{n}\right) \in \Omega_{n-1} \times \Omega_{n}^{0-} .
\end{gathered}
$$


Analogously,

$$
\begin{gathered}
\psi_{n}^{2}\left(\omega_{1}, \ldots, \omega_{n-1}, \omega_{n}\right)= \\
\int_{\Omega_{n}^{0}} \chi_{\Omega_{n}^{0-}}\left(\omega_{n}^{1}\right) \alpha_{n}\left(\left\{\omega_{1}, \ldots, \omega_{n-1}, \omega_{n}^{1}\right\} ;\left\{\omega_{1}, \ldots, \omega_{n-1}, \omega_{n}\right\}\right) \times \\
\frac{\Delta S_{n}^{-}\left(\omega_{1}, \ldots, \omega_{n-1}, \omega_{n}^{1}\right)}{V_{n}\left(\omega_{1}, \ldots, \omega_{n-1}, \omega_{n}^{1}, \omega_{n}^{2}\right)} d P_{n}^{0}\left(\omega_{n}^{1}\right)= \\
S_{n-1}\left(\omega_{1}, \ldots, \omega_{n-1}\right) \alpha_{n}^{2}\left(\omega_{n}\right) \int_{\Omega_{n}^{0}} \chi_{\Omega_{n}^{0-}}\left(\omega_{n}^{1}\right) \alpha_{n}^{1}\left(\omega_{n}^{1}\right) \times \\
\frac{a_{n}\left(\omega_{1}, \ldots, \omega_{n-1}, \omega_{n}^{1}\right) \eta_{n}^{-}\left(\omega_{n}^{1}\right)}{V_{n}\left(\omega_{1}, \ldots, \omega_{n-1}, \omega_{n}^{1}, \omega_{n}^{2}\right)} d P_{n}^{0}\left(\omega_{n}^{1}\right)>0, \quad\left(\omega_{1}, \ldots, \omega_{n}\right) \in \Omega_{n-1} \times \Omega_{n}^{0+} .
\end{gathered}
$$

From these inequalities, we obtain

$$
\psi_{n}\left(\omega_{1}, \ldots, \omega_{n-1}, \omega_{n}\right)>0, \quad\left(\omega_{1}, \ldots, \omega_{n}\right) \in \Omega_{n} .
$$

This proves the equivalence of the measures $P_{N}$ and $\mu_{0}$.

Theorem 5. Suppose that the conditions of Lemma 2 are true. Then, the set of strictly positive random values $\alpha_{n}\left(\{\omega\}_{n}^{1} ;\{\omega\}_{n}^{2}\right), n=\overline{1, N}$, satisfying the conditions

$$
\begin{gathered}
E^{\mu_{0}}\left|\Delta S_{n}\left(\omega_{1}, \ldots, \omega_{n-1}, \omega_{n}\right)\right|= \\
\int_{\Omega_{N}} \prod_{i=1}^{N} \psi_{i}\left(\omega_{1}, \ldots, \omega_{i}\right)\left|\Delta S_{n}\left(\omega_{1}, \ldots, \omega_{n-1}, \omega_{n}\right)\right| \prod_{i=1}^{N} d P_{i}^{0}\left(\omega_{i}\right)<\infty, \quad n=\overline{1, N}
\end{gathered}
$$

is a nonempty one and the convex linear span of the set of measures (104), defined by the random values $\alpha_{n}\left(\left\{\omega_{1}^{1}, \ldots, \omega_{n}^{1}\right\} ;\left\{\omega_{1}^{2}, \ldots, \omega_{n}^{2}\right\}\right), n=\overline{1, N}$, satisfying the conditions (111), is a set of martingale measures being equivalent to the measure $P_{N}$.

Proof. All bounded random values $\alpha_{n}\left(\left\{\omega_{1}^{1}, \ldots, \omega_{n}^{1}\right\} ;\left\{\omega_{1}^{2}, \ldots, \omega_{n}^{2}\right\}\right), n=\overline{1, N}$, constructed in Lemma 2 satisfy the conditions (111), since $\left|\Delta S_{n}\left(\omega_{1}, \ldots, \omega_{n-1}, \omega_{n}\right)\right|$ takes only finite values. The fact that the measures (104) are martingale ones is proved as early (see [1]).

Lemma 4. Suppose that the conditions of Lemma 2 are valid. If, on the probability space $\left\{\Omega_{n-1}, \mathcal{F}_{n-1}, \mu_{0}^{n-1}\right\}$, for each $B \in \mathcal{F}_{n-1}, \mu_{0}^{n-1}(B)>0$, the nonnegative random value $f_{n}\left(\omega_{1}, \ldots, \omega_{n-1}, \omega_{n}\right)$ satisfies the inequality

$$
\frac{1}{\mu_{0}^{n-1}(B)} \int_{B} \int_{\Omega_{n}^{0}} \prod_{i=1}^{n} \psi_{i}\left(\omega_{1}, \ldots, \omega_{i}\right) f_{n}\left(\omega_{1}, \ldots, \omega_{n}\right) \prod_{i=1}^{n} d P_{i}^{0}\left(\omega_{i}\right) \leq 1, \quad B \in \mathcal{F}_{n-1}
$$


then the inequality

$$
\begin{gathered}
\int_{\Omega_{n}^{0}} \psi_{n}\left(\omega_{1}, \ldots, \omega_{n}\right) f_{n}\left(\omega_{1}, \ldots, \omega_{n}\right) d P_{n}^{0}\left(\omega_{n}\right) \leq 1, \\
\left\{\omega_{1}, \ldots, \omega_{n-1}\right\} \in \Omega_{n-1}, n=\overline{1, N}
\end{gathered}
$$

is true.

Proof. The proof see in [1].

Theorem 6. Suppose that for $\Delta S_{n}\left(\omega_{1}, \ldots, \omega_{n-1}, \omega_{n}\right), n=\overline{1, N}$, the representation (13) is valid and Lemma 4 conditions are true. Then, for the nonnegative random value $f_{n}\left(\omega_{1}, \ldots, \omega_{n-1}, \omega_{n}\right)$ the inequalities

$$
\begin{gathered}
\chi_{\Omega_{n}^{0-}}\left(\omega_{n}^{1}\right) \chi_{\Omega_{n}^{0+}}\left(\omega_{n}^{2}\right)\left[\frac{\Delta S_{n}^{+}\left(\omega_{1}, \ldots, \omega_{n-1}, \omega_{n}^{2}\right)}{V_{n}\left(\omega_{1}, \ldots, \omega_{n-1}, \omega_{n}^{1}, \omega_{n}^{2}\right)} f_{n}\left(\omega_{1}, \ldots, \omega_{n-1}, \omega_{n}^{1}\right)+\right. \\
\left.\frac{\Delta S_{n}^{-}\left(\omega_{1}, \ldots, \omega_{n-1}, \omega_{n}^{1}\right)}{V_{n}\left(\omega_{1}, \ldots, \omega_{n-1}, \omega_{n}^{1}, \omega_{n}^{2}\right)} f_{n}\left(\omega_{1}, \ldots, \omega_{n-1}, \omega_{n}^{2}\right)\right] \leq 1, \\
\left(\omega_{1}, \ldots, \omega_{n-1}\right) \in \Omega_{n-1}, \quad\left(\omega_{n}^{1}, \omega_{n}^{2}\right) \in \Omega_{n}^{0-} \times \Omega_{n}^{0+}, \quad n=\overline{1, N},
\end{gathered}
$$

are true.

Proof. The proof see in [1].

Theorem 7. Suppose that the conditions of Theorem 6 are true. Then, the nonnegative random values $f_{n}\left(\omega_{1}, \ldots, \omega_{n-1}, \omega_{n}\right), n=\overline{1, N}$, satisfy the inequalities

$$
\begin{gathered}
f_{n}\left(\omega_{1}, \ldots, \omega_{n-1}, \omega_{n}\right) \leq \\
\left(1+\gamma_{n-1}\left(\omega_{1}, \ldots, \omega_{n-1}\right) \Delta S_{n}\left(\omega_{1}, \ldots, \omega_{n-1}, \omega_{n}\right)\right), \quad n=\overline{1, N},
\end{gathered}
$$

where $\gamma_{n-1}\left(\omega_{1}, \ldots, \omega_{n-1}\right)$ is a bounded $\mathcal{F}_{n-1}$-measurable random value.

Proof. It is evident that there exists $\omega_{n}^{1} \in \Omega_{n}^{0-}$ and $\omega_{n}^{2} \in \Omega_{n}^{0+}$ such that the inequalities

$$
\begin{gathered}
\max _{\left(\omega_{1}, \ldots, \omega_{n-1}\right) \in \Omega_{n-1}} \frac{1}{\Delta S_{n}^{-}\left(\omega_{1}, \ldots, \omega_{n-1}, \omega_{n}^{1}\right)}<\infty \\
\max _{\left(\omega_{1}, \ldots, \omega_{n-1}\right) \in \Omega_{n-1}} \frac{1}{\Delta S_{n}^{+}\left(\omega_{1}, \ldots, \omega_{n-1}, \omega_{n}^{2}\right)}<\infty, \quad n=\overline{1, N},
\end{gathered}
$$

are true. This proves Theorem 7 (see [1]).

Theorem 8. Suppose that the evolution $\left\{S_{n}\left(\omega_{1}, \ldots, \omega_{n}\right)\right\}_{n=1}^{N}$ of risky asset satisfies the conditions of Theorems 5, 6, 7, then for every nonnegative super-martingale $\left\{f_{n}^{1}\left(\omega_{1}, \ldots, \omega_{n}\right)\right\}_{n=0}^{N}$ relative to the set of martingale measure $M$, described in Theorem 5, the optional decomposition is true. 
Proof. The proof see in [1]. More detail about optional decomposition see in [25], [26], [28] [27], [29].

Let us consider the random values

$$
\begin{gathered}
\psi_{n}\left(\omega_{1}, \ldots, \omega_{n}\right)=\chi_{\Omega_{n}^{0-}}\left(\omega_{n}\right) \psi_{n}^{1}\left(\omega_{1}, \ldots, \omega_{n}\right)+ \\
\chi_{\Omega_{n}^{0+}}\left(\omega_{n}\right) \psi_{n}^{2}\left(\omega_{1}, \ldots, \omega_{n}\right)
\end{gathered}
$$

where

$$
\begin{gathered}
\psi_{n}^{1}\left(\omega_{1}, \ldots, \omega_{n-1}, \omega_{n}\right)= \\
\frac{\Delta S_{n}^{+}\left(\omega_{1}, \ldots, \omega_{n-1}, \omega_{n}^{2}\right)}{V_{n}\left(\omega_{1}, \ldots, \omega_{n-1}, \omega_{n}^{1}, \omega_{n}^{2}\right)}, \quad\left(\omega_{1}, \ldots, \omega_{n-1}\right) \in \Omega_{n-1}, \\
\psi_{n}^{2}\left(\omega_{1}, \ldots, \omega_{n-1}, \omega_{n}\right)= \\
\frac{\Delta S_{n}^{-}\left(\omega_{1}, \ldots, \omega_{n-1}, \omega_{n}^{1}\right)}{V_{n}\left(\omega_{1}, \ldots, \omega_{n-1}, \omega_{n}^{1}, \omega_{n}^{2}\right)}, \quad\left(\omega_{1}, \ldots, \omega_{n-1}\right) \in \Omega_{n-1}, \quad n=\overline{1, N} .
\end{gathered}
$$

Definition 1. Let the evolution of risky assets be given by the formula (8). On the measurable space $\left\{\Omega_{N}, \mathcal{F}_{N}\right\}$, being the direct product of the measurable spaces $\left\{\Omega_{i}^{0}, \mathcal{F}_{i}^{0}\right\}$, for every point $\left\{\left\{\omega_{1}^{1}, \omega_{1}^{2}\right\}, \ldots,\left\{\omega_{N}^{1}, \omega_{N}^{2}\right\}\right\} \in \prod_{i=1}^{N}\left[\Omega_{i}^{0-} \times \Omega_{i}^{0+}\right]$ let us introduce the spot measure

$$
\begin{gathered}
\mu_{\left\{\omega_{1}^{1}, \omega_{1}^{2}\right\}, \ldots,\left\{\omega_{N}^{1}, \omega_{N}^{2}\right\}}(A)= \\
\sum_{i_{1}=1}^{2} \ldots \sum_{i_{N}=1}^{2} \prod_{n=1}^{N} \psi_{n}\left(\omega_{1}^{i_{1}}, \ldots, \omega_{n}^{i_{n}}\right) \chi_{A}\left(\omega_{1}^{i_{1}}, \ldots, \omega_{N}^{i_{N}}\right), \quad A \in \mathcal{F}_{N},
\end{gathered}
$$

where $\psi_{n}\left(\omega_{1}, \ldots, \omega_{n}\right)$ is determined by the formulas (117) - (119).

Lemma 5. The spot measure $\mu_{\left\{\omega_{1}^{1}, \omega_{1}^{2}\right\}, \ldots,\left\{\omega_{N}^{1}, \omega_{N}^{2}\right\}}(A)$, given by the formula (120), is a martingale measure for the evolution of risky asset given by the formula (8) for every point $\left\{\left\{\omega_{1}^{1}, \omega_{1}^{2}\right\}, \ldots,\left\{\omega_{N}^{1}, \omega_{N}^{2}\right\}\right\} \in \prod_{i=1}^{N}\left[\Omega_{i}^{0-} \times \Omega_{i}^{0+}\right]$. If the point $\left\{\omega_{1}^{1}, \omega_{1}^{2}\right\}, \ldots,\left\{\omega_{N}^{1}, \omega_{N}^{2}\right\}$ is such that $\Delta S_{n}\left(\omega_{1}, \ldots, \omega_{n-1}, \omega_{n}^{1}\right)<0, \Delta S_{n}\left(\omega_{1}, \ldots, \omega_{n-1} \omega_{n}^{2}\right)>0,\left\{\omega_{1}, \ldots, \omega_{n-1}\right\} \in$ $\Omega_{n-1}, n=\overline{1, N}$, then the spot measure $\mu_{\left\{\omega_{1}^{1}, \omega_{1}^{2}\right\}, \ldots,\left\{\omega_{N}^{1}, \omega_{N}^{2}\right\}}(A)$ is a martingale measure being equivalent to the measure $P_{N}$.

Proof. Let us prove that $\mu_{\left\{\omega_{1}^{1}, \omega_{1}^{2}\right\}, \ldots,\left\{\omega_{N}^{1}, \omega_{N}^{2}\right\}}(A)$ is a probability measure. Let us calculate

$$
\sum_{i_{j}=1}^{2} \psi_{j}\left(\omega_{1}^{i_{1}}, \ldots, \omega_{j}^{i_{j}}\right)=\psi_{j}\left(\omega_{1}^{i_{1}}, \ldots, \omega_{j-1}^{i_{j-1}}, \omega_{j}^{1}\right)+\psi_{j}\left(\omega_{1}^{i_{1}}, \ldots, \omega_{j-1}^{i_{j-1}}, \omega_{j}^{2}\right)=
$$




$$
\begin{aligned}
& \chi_{\Omega_{j}^{0-}}\left(\omega_{j}^{1}\right) \psi_{j}^{1}\left(\omega_{1}^{i_{1}}, \ldots, \omega_{j-1}^{i_{j-1}} \omega_{j}^{1}\right)+ \\
& \chi_{\Omega_{j}^{0-}}\left(\omega_{j}^{1}\right) \psi_{j}^{1}\left(\omega_{1}^{i_{1}}, \ldots, \omega_{j-1}^{i_{j-1}} \omega_{j}^{1}\right)+ \\
& \chi_{\Omega_{j}^{0+}}\left(\omega_{j}^{1}\right) \psi_{j}^{2}\left(\omega_{1}^{i_{1}}, \ldots, \omega_{j-1}^{i_{j-1}} \omega_{j}^{1}\right)+ \\
& \chi_{\Omega_{j}^{0-}}\left(\omega_{j}^{2}\right) \psi_{j}^{1}\left(\omega_{1}^{i_{1}}, \ldots, \omega_{j-1}^{i_{j-1}} \omega_{j}^{2}\right)+ \\
& \chi_{\Omega_{j}^{0+}}\left(\omega_{j}^{2}\right) \psi_{j}^{2}\left(\omega_{1}^{i_{1}}, \ldots, \omega_{j-1}^{i_{j-1}} \omega_{j}^{2}\right)= \\
& \chi_{\Omega_{j}^{0-}}\left(\omega_{j}^{1}\right) \chi_{\Omega_{j}^{0+}}\left(\omega_{j}^{2}\right) \frac{\Delta S_{j}^{+}\left(\omega_{1}^{i_{1}}, \ldots, \omega_{j-1}^{j-1}, \omega_{j}^{2}\right)}{V_{j}\left(\omega_{1}^{i_{1}}, \ldots, \omega_{j-1}^{i_{j-1}}, \omega_{j}^{1}, \omega_{j}^{2}\right)}+ \\
& \chi_{\Omega_{j}^{0+}}\left(\omega_{j}^{1}\right) \chi_{\Omega_{j}^{0-}}\left(\omega_{j}^{1}\right) \frac{\Delta S_{j}^{-}\left(\omega_{1}^{i_{1}}, \ldots, \omega_{j-1}^{i_{j-1}}, \omega_{j}^{1}\right)}{V_{j}\left(\omega_{1}^{i_{1}}, \ldots, \omega_{j-1}^{i_{j-1}}, \omega_{j}^{1}, \omega_{j}^{1}\right)}+ \\
& \chi_{\Omega_{j}^{0-}}\left(\omega_{j}^{2}\right) \chi_{\Omega_{j}^{0+}}\left(\omega_{j}^{2}\right) \frac{\Delta S_{j}^{+}\left(\omega_{1}^{i_{1}}, \ldots, \omega_{j-1}^{i_{j-1}}, \omega_{j}^{2}\right)}{V_{j}\left(\omega_{1}^{i_{1}}, \ldots, \omega_{j-1}^{i_{j-1}}, \omega_{j}^{1}, \omega_{j}^{2}\right)}+ \\
& \chi_{\Omega_{j}^{0+}}\left(\omega_{j}^{2}\right) \chi_{\Omega_{j}^{0-}}\left(\omega_{j}^{1}\right) \frac{\Delta S_{j}^{-}\left(\omega_{1}^{i_{1}}, \ldots, \omega_{j-1}^{i_{j-1}}, \omega_{j}^{1}\right)}{V_{j}\left(\omega_{1}^{i_{1}}, \ldots, \omega_{j-1}^{i_{j-1}}, \omega_{j}^{1}, \omega_{j}^{1}\right)}= \\
& \chi_{\Omega_{j}^{0-}}\left(\omega_{j}^{1}\right) \chi_{\Omega_{j}^{0+}}\left(\omega_{j}^{2}\right) \frac{\Delta S_{j}^{+}\left(\omega_{1}^{i_{1}}, \ldots, \omega_{j-1}^{i_{j-1}}, \omega_{j}^{2}\right)}{V_{j}\left(\omega_{1}^{i_{1}}, \ldots, \omega_{j-1}^{i_{j-1}}, \omega_{j}^{1}, \omega_{j}^{2}\right)}+ \\
& \chi_{\Omega_{j}^{0+}}\left(\omega_{j}^{2}\right) \chi_{\Omega_{j}^{0-}}\left(\omega_{j}^{1}\right) \frac{\Delta S_{j}^{-}\left(\omega_{1}^{i_{1}}, \ldots, \omega_{j-1}^{i_{j-1}}, \omega_{j}^{1}\right)}{V_{j}\left(\omega_{1}^{i_{1}}, \ldots, \omega_{j-1}^{i_{j-1}}, \omega_{j}^{1}, \omega_{j}^{1}\right)}=\chi_{\Omega_{j}^{0-}}\left(\omega_{j}^{1}\right) \chi_{\Omega_{j}^{0+}}\left(\omega_{j}^{2}\right)=1 .
\end{aligned}
$$

The last equalities prove that $\mu_{\left\{\omega_{1}^{1}, \omega_{1}^{2}\right\}, \ldots,\left\{\omega_{N}^{1}, \omega_{N}^{2}\right\}}\left(\Omega_{N}\right)=1$ for every point $\left\{\left\{\omega_{1}^{1}, \omega_{1}^{2}\right\}, \ldots,\left\{\omega_{N}^{1}, \omega_{N}^{2}\right\}\right\} \in \prod_{i=1}^{N}\left[\Omega_{i}^{0-} \times \Omega_{i}^{0+}\right]$. Further

$$
\begin{gathered}
\sum_{i_{j}=1}^{2} \psi_{j}\left(\omega_{1}^{i_{1}}, \ldots, \omega_{j}^{i_{j}}\right) \Delta S_{j}\left(\omega_{1}^{i_{1}}, \ldots, \omega_{j}^{i_{j}}\right)= \\
\psi_{j}\left(\omega_{1}^{i_{1}}, \ldots, \omega_{j-1}^{i_{j-1}}, \omega_{j}^{1}\right) \Delta S_{j}\left(\omega_{1}^{i_{1}}, \ldots, \omega_{j-1}^{i_{j-1}}, \omega_{j}^{1}\right)+ \\
\psi_{j}\left(\omega_{1}^{i_{1}}, \ldots, \omega_{j-1}^{i_{j-1}}, \omega_{j}^{2}\right) \Delta S_{j}\left(\omega_{1}^{i_{1}}, \ldots, \omega_{j-1}^{i_{j-1}}, \omega_{j}^{2}\right)= \\
\chi_{\Omega_{j}^{0-}}\left(\omega_{j}^{1}\right) \chi_{\Omega_{j}^{0+}}\left(\omega_{j}^{2}\right) \times
\end{gathered}
$$




$$
\begin{gathered}
{\left[-\frac{\Delta S_{j}^{+}\left(\omega_{1}^{i_{1}}, \ldots, \omega_{j-1}^{i_{j-1}}, \omega_{j}^{2}\right)}{V_{j}\left(\omega_{1}^{i_{1}}, \ldots, \omega_{j-1}^{i_{j-1}}, \omega_{j}^{1}, \omega_{j}^{2}\right)} \Delta S_{j}^{-}\left(\omega_{1}^{i_{1}}, \ldots, \omega_{j-1}^{i_{j-1}}, \omega_{j}^{1}\right)+\right.} \\
\left.\frac{\Delta S_{j}^{-}\left(\omega_{1}^{i_{1}}, \ldots, \omega_{j-1}^{i_{j-1}}, \omega_{j}^{1}\right)}{V_{j}\left(\omega_{1}^{i_{1}}, \ldots, \omega_{j-1}^{i_{j-1}}, \omega_{j}^{1}, \omega_{j}^{1}\right)} \Delta S_{j}^{+}\left(\omega_{1}^{i_{1}}, \ldots, \omega_{j-1}^{i_{j-1}}, \omega_{j}^{2}\right)\right]=0, \quad j=\overline{1, N} .
\end{gathered}
$$

Let us prove that the set of measures $\mu_{\left\{\omega_{1}^{1}, \omega_{1}^{2}\right\}, \ldots,\left\{\omega_{N}^{1}, \omega_{N}^{2}\right\}}(A)$ is a set of martingale measures. Really, for $A$, belonging to the $\sigma$-algebra $\mathcal{F}_{n-1}$ of the filtration we have $A=B \times \prod_{i=n}^{N} \Omega_{i}^{0}$, where $B$ belongs to $\sigma$-algebra $\mathcal{F}_{n-1}$ of the measurable space $\left\{\Omega_{n-1}, \mathcal{F}_{n-1}\right\}$. Then,

$$
\begin{gathered}
\int_{A} \Delta S_{n}\left(\omega_{1}, \ldots, \omega_{n}\right) d \mu_{\left\{\omega_{1}^{1}, \omega_{1}^{2}\right\}, \ldots,\left\{\omega_{N}^{1}, \omega_{N}^{2}\right\}}= \\
\sum_{i_{1}=1}^{2} \ldots \sum_{i_{N}=1}^{2} \prod_{j=1}^{N} \psi_{j}\left(\omega_{1}^{i_{1}}, \ldots, \omega_{j}^{i_{j}}\right) \chi_{B}\left(\omega_{1}^{i_{1}}, \ldots, \omega_{n-1}^{i_{n-1}}\right) \Delta S_{n}\left(\omega_{1}^{i_{1}}, \ldots, \omega_{n}^{i_{n}}\right)= \\
\sum_{i_{1}=1}^{2} \ldots \sum_{i_{n}=1}^{2} \prod_{j=1}^{n} \psi_{j}\left(\omega_{1}^{i_{1}}, \ldots, \omega_{j}^{i_{j}}\right) \chi_{B}\left(\omega_{1}^{i_{1}}, \ldots, \omega_{n-1}^{i_{n-1}}\right) \Delta S_{n}\left(\omega_{1}^{i_{1}}, \ldots, \omega_{n}^{i_{n}}\right)= \\
\sum_{i_{1}=1}^{2} \ldots \sum_{i_{n-1}=1}^{2} \prod_{j=1}^{n-1} \psi_{j}\left(\omega_{1}^{i_{1}}, \ldots, \omega_{j}^{i_{j}}\right) \chi_{B}\left(\omega_{1}^{i_{1}}, \ldots, \omega_{n-1}^{i_{n-1}}\right) \times \\
\sum_{i_{n}=1}^{2} \psi_{n}\left(\omega_{1}^{i_{1}}, \ldots, \omega_{n}^{i_{n}}\right) \Delta S_{n}\left(\omega_{1}^{i_{1}}, \ldots, \omega_{n}^{i_{n}}\right)=0, \quad A \in \mathcal{F}_{n-1} .
\end{gathered}
$$

To prove the last statement it needs to prove that $\psi_{n}\left(\omega_{1}, \ldots, \omega_{n}\right)>0, n=\overline{1, N}$. But,

$$
\begin{gathered}
\psi_{n}\left(\omega_{1}, \ldots, \omega_{n}\right)=\chi_{\Omega_{n}^{0-}}\left(\omega_{n}\right) \frac{\Delta S_{n}^{+}\left(\omega_{1}, \ldots, \omega_{n-1}, \omega_{n}^{2}\right)}{V_{n}\left(\omega_{1}, \ldots, \omega_{n-1}, \omega_{n}^{1}, \omega_{n}^{2}\right)}+ \\
\chi_{\Omega_{n}^{0+}}\left(\omega_{n}\right) \frac{\Delta S_{n}^{-}\left(\omega_{1}, \ldots, \omega_{n-1}, \omega_{n}^{1}\right)}{V_{n}\left(\omega_{1}, \ldots, \omega_{n-1}, \omega_{n}^{1}, \omega_{n}^{2}\right)}>0, \quad n=\overline{1, N} .
\end{gathered}
$$

The last means the needed statement.

We remind that the evolution of risky asset is given by the formula (8). Therefore, in this case the condition (16) (see [1]) is formulated, as follows:

$$
\int_{\Omega_{n}^{0} \times \Omega_{n}^{0}} \chi_{\Omega_{n}^{0-}}\left(\omega_{n}^{1}\right) \chi_{\Omega_{n}^{0+}}\left(\omega_{n}^{2}\right) \alpha_{n}\left(\left\{\omega_{1}^{1}, \ldots, \omega_{n-1}^{1}, \omega_{n}^{1}\right\} ;\left\{\omega_{1}^{2}, \ldots, \omega_{n-1}^{2}, \omega_{n}^{2}\right\}\right) \times
$$




$$
d P_{n}^{0}\left(\omega_{n}^{1}\right) d P_{n}^{0}\left(\omega_{n}^{2}\right)=1, \quad n=\overline{1, N}
$$

Below, we describe the convex set of equivalent martingale measures.

Theorem 9. The measure $\mu_{0}(A)$, constructed by the strictly positive finite valued random values $\alpha_{n}\left(\{\omega\}_{n}^{1} ;\{\omega\}_{n}^{2}\right), n=\overline{1, N}$, satisfying the conditions (124), is a martingale measure for the evolution of risky asset, given by the formula (8). Every measure, belonging to the convex linear span of such measures, is also martingale measure for the considered evolution of risky asset. They are equivalent to the measure $P_{N}$.

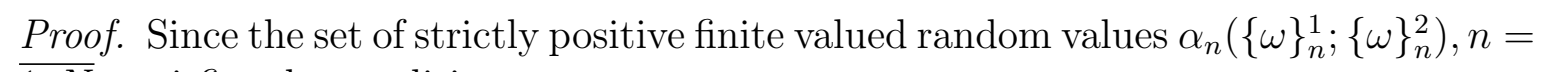
$\overline{1, N}$, satisfies the conditions

$$
\begin{gathered}
E^{\mu_{0}}\left|\Delta S_{n}\left(\omega_{1}, \ldots, \omega_{n-1}, \omega_{n}\right)\right|= \\
\int_{\Omega_{N}} \prod_{i=1}^{N} \psi_{i}\left(\omega_{1}, \ldots, \omega_{i}\right)\left|\Delta S_{n}\left(\omega_{1}, \ldots, \omega_{n-1}, \omega_{n}\right)\right| \prod_{i=1}^{N} d P_{i}^{0}\left(\omega_{i}\right)<\infty, \quad n=\overline{1, N}
\end{gathered}
$$

then the set of measures $\mu_{0}(A)$, given by the formula (111), is a non empty one. This proves Theorem 9 .

We use for $\alpha_{N}\left(\left\{\omega_{1}^{1}, \ldots, \omega_{N}^{1}\right\} ;\left\{\omega_{1}^{2}, \ldots, \omega_{N}^{2}\right\}\right)$ the denotation $\alpha_{N}\left(\{\omega\}_{N}^{1} ;\{\omega\}_{N}^{2}\right)$.

Theorem 10. Let the evolution of risky asset be given by the formula (8). On the measurable space with measure $\left\{\prod_{i=1}^{N}\left[\Omega_{i}^{0-} \times \Omega_{i}^{0+}\right], \prod_{i=1}^{N}\left[\mathcal{F}_{i}^{0-} \times \mathcal{F}_{i}^{0+}\right], \prod_{i=1}^{N}\left[P_{i}^{0-} \times P_{i}^{0+}\right]\right\}$, suppose that the random value $\alpha_{N}\left(\{\omega\}_{N}^{1} ;\{\omega\}_{N}^{2}\right)$, satisfies the conditions

$$
\begin{aligned}
& \alpha_{N}\left(\{\omega\}_{N}^{1} ;\{\omega\}_{N}^{2}\right)>0, \quad\left\{\omega_{1}^{1}, \omega_{1}^{2}\right\}, \ldots,\left\{\omega_{N}^{1}, \omega_{N}^{2}\right\} \in \prod_{i=1}^{N}\left[\Omega_{i}^{0-} \times \Omega_{i}^{0+}\right], \\
& \quad \int_{\prod_{i=1}^{N}\left[\Omega_{i}^{0-} \times \Omega_{i}^{0+}\right]} \alpha_{N}\left(\left\{\omega_{1}^{1}, \ldots, \omega_{N}^{1}\right\} ;\left\{\omega_{1}^{2}, \ldots, \omega_{N}^{2}\right\}\right) \prod_{i=1}^{N} d P_{i}^{0}\left(\omega_{i}^{1}\right) d P_{i}^{0}\left(\omega_{i}^{2}\right)=1 .
\end{aligned}
$$

The measure $\mu_{0}(A)$, given by the formula

$$
\begin{gathered}
\mu_{0}(A)= \\
\int_{\prod_{i=1}^{N}\left[\Omega_{i}^{0-} \times \Omega_{i}^{0+}\right]} \alpha_{N}\left(\{\omega\}_{N}^{1} ;\{\omega\}_{N}^{2}\right) \mu_{\left\{\omega_{1}^{1}, \omega_{1}^{2}\right\}, \ldots,\left\{\omega_{N}^{1}, \omega_{N}^{2}\right\}}(A) d \prod_{i=1}^{N}\left[P_{i}^{0-} \times P_{i}^{0+}\right],
\end{gathered}
$$

is a martingale measure, being equivalent to the measure $P_{N}$. 
Proof. Let us note that $\mu_{\left\{\omega_{1}^{1}, \omega_{1}^{2}\right\}, \ldots,\left\{\omega_{N}^{1}, \omega_{N}^{2}\right\}}\left(\Omega_{N}\right)=0$ if $\left\{\left\{\omega_{1}^{1}, \omega_{1}^{2}\right\}, \ldots,\left\{\omega_{N}^{1}, \omega_{N}^{2}\right\}\right\}$ does not belong to the set $\prod_{i=1}^{N}\left[\Omega_{i}^{0-} \times \Omega_{i}^{0+}\right]$. Let us introduce the denotations

$$
\alpha_{n}\left(\left\{\omega_{1}^{1}, \ldots, \omega_{n-1}^{1}, \omega_{n}^{1}\right\} ;\left\{\omega_{1}^{2}, \ldots, \omega_{n-1}^{2}, \omega_{n}^{2}\right\}\right)=
$$

$$
\begin{aligned}
& \int_{N} \alpha_{N}\left(\left\{\omega_{1}^{1}, \ldots, \omega_{N}^{1}\right\} ;\left\{\omega_{1}^{2}, \ldots, \omega_{N}^{2}\right\}\right) \prod_{i=n+1}^{N} d P_{i}^{0}\left(\omega_{i}^{1}\right) d P_{i}^{0}\left(\omega_{i}^{2}\right) \\
& \frac{\prod_{i=n+1}^{N}\left[\Omega_{i}^{0-} \times \Omega_{i}^{0+}\right]}{\int \alpha_{N}\left(\left\{\omega_{1}^{1}, \ldots, \omega_{N}^{1}\right\} ;\left\{\omega_{1}^{2}, \ldots, \omega_{N}^{2}\right\}\right) \prod_{i=n}^{N} d P_{i}^{0}\left(\omega_{i}^{1}\right) d P_{i}^{0}\left(\omega_{i}^{2}\right)}, n=\overline{1, N} \text {. }
\end{aligned}
$$
$\prod_{i=n}^{N}\left[\Omega_{i}^{0-} \times \Omega_{i}^{0+}\right]$

Since the random values $\alpha_{n}\left(\left\{\omega_{1}^{1}, \ldots, \omega_{n-1}^{1}, \omega_{n}^{1}\right\} ;\left\{\omega_{1}^{2}, \ldots, \omega_{n-1}^{2}, \omega_{n}^{2}\right\}\right)$ are finite valued, then

$$
\begin{gathered}
\int_{\Omega_{n}^{0-} \times \Omega_{n}^{0+}} \alpha_{n}\left(\left\{\omega_{1}^{1}, \ldots, \omega_{n-1}^{1}, \omega_{n}^{1}\right\} ;\left\{\omega_{1}^{2}, \ldots, \omega_{n-1}^{2}, \omega_{n}^{2}\right\}\right) \times \\
\frac{\Delta S_{n}^{+}\left(\omega_{1}, \ldots, \omega_{n-1}, \omega_{n}^{2}\right) \Delta S_{n}^{-}\left(\omega_{1}, \ldots, \omega_{n-1}, \omega_{n}^{1}\right)}{V_{n}\left(\omega_{1}, \ldots, \omega_{n-1}, \omega_{n}^{1}, \omega_{n}^{2}\right)} d P_{n}^{0}\left(\omega_{n}^{1}\right) d P_{n}^{0}\left(\omega_{n}^{2}\right)<\infty \\
\left(\omega_{1}, \ldots, \omega_{n-1}\right) \in \Omega_{n-1} .
\end{gathered}
$$

It is evident that the set of strictly positive finite valued random values $\alpha_{n}\left(\{\omega\}_{n}^{1} ;\{\omega\}_{n}^{2}\right), n=\overline{1, N}$, given by the formula (129), satisfy the conditions

$$
\begin{gathered}
E^{\mu_{0}}\left|\Delta S_{n}\left(\omega_{1}, \ldots, \omega_{n-1}, \omega_{n}\right)\right|= \\
\int_{\Omega_{N}} \prod_{i=1}^{N} \psi_{i}\left(\omega_{1}, \ldots, \omega_{i}\right)\left|\Delta S_{n}\left(\omega_{1}, \ldots, \omega_{n-1}, \omega_{n}\right)\right| \prod_{i=1}^{N} d P_{i}^{0}\left(\omega_{i}\right)<\infty, \quad n=\overline{1, N} .
\end{gathered}
$$

Moreover, for the measure (128) the representation (104) is true, meaning that it is equivalent to the measure $P_{N}$. The last proves Theorem 10 .

Let us define the integral for the random value $f_{N}\left(\omega_{1}, \ldots, \omega_{N-1}, \omega_{N}\right)$ relative to the measure $\mu_{\left\{\omega_{1}^{1}, \omega_{1}^{2}\right\}, \ldots,\left\{\omega_{N}^{1}, \omega_{N}^{2}\right\}}(A)$ by the formula

$$
\begin{array}{r}
\int_{\Omega_{N}} f_{N}\left(\omega_{1}, \ldots, \omega_{N-1}, \omega_{N}\right) d \mu_{\left\{\omega_{1}^{1}, \omega_{1}^{2}\right\}, \ldots,\left\{\omega_{N}^{1}, \omega_{N}^{2}\right\}}= \\
\sum_{i_{1}=1}^{2} \ldots \sum_{i_{N}=1}^{2} \prod_{n=1}^{N} \psi_{n}\left(\omega_{1}^{i_{1}}, \ldots, \omega_{n}^{i_{n}}\right) f_{N}\left(\omega_{1}^{i_{1}}, \ldots, \omega_{N}^{i_{N}}\right) .
\end{array}
$$


Theorem 11. Let the evolution of risky asset be given by the formula (8). If the conditions of Theorem 10 are true, then the fair price of super-hedge for the nonnegative payoff function $f(x)$ is given by the formula

$$
f_{0}=\sup _{P \in M} E^{P} f\left(S_{N}\right)=\max _{\omega_{i}^{1} \in \Omega_{i}^{0-}, \omega_{i}^{2} \in \Omega_{i}^{0+}, i=\overline{1, N}} \int_{\Omega_{N}} f\left(S_{N}\right) d \mu_{\left\{\omega_{1}^{1}, \omega_{1}^{2}\right\}, \ldots,\left\{\omega_{N}^{1}, \omega_{N}^{2}\right\}} .
$$

Proof. Let us prove the formula (133). Denote $M$ the set of all martingale measures, being equivalent to $P_{N}$. If an equivalent martingale measure $P_{0} \in M$, then $\alpha P_{0}+$ $(1-\alpha) \mu_{\left\{\omega_{1}^{1}, \omega_{1}^{2}\right\}, \ldots,\left\{\omega_{N}^{1}, \omega_{N}^{2}\right\}} \in M$ for arbitrary $0<\alpha \leq 1$. We have the inequality

$$
\alpha E^{P_{0}} f\left(S_{N}\right)+(1-\alpha) \int_{\Omega_{N}} f\left(S_{N}\right) d \mu_{\left\{\omega_{1}^{1}, \omega_{1}^{2}\right\}, \ldots,\left\{\omega_{N}^{1}, \omega_{N}^{2}\right\}} \leq \sup _{P \in M} E^{P} f\left(S_{N}\right) .
$$

Since $\alpha>0$ is arbitrary, we obtain the inequality

$$
\int_{\Omega_{N}} f\left(S_{N}\right) d \mu_{\left\{\omega_{1}^{1}, \omega_{1}^{2}\right\}, \ldots,\left\{\omega_{N}^{1}, \omega_{N}^{2}\right\}} \leq \sup _{P \in M} E^{P} f\left(S_{N}\right) .
$$

From here, we obtain the inequality

$$
\max _{\omega_{i}^{1} \in \Omega_{i}^{0-}, \omega_{i}^{2} \in \Omega_{i}^{0+}, i=\overline{1, N}} \int_{\Omega_{N}} f\left(S_{N}\right) d \mu_{\left\{\omega_{1}^{1}, \omega_{1}^{2}\right\}, \ldots,\left\{\omega_{N}^{1}, \omega_{N}^{2}\right\}} \leq \sup _{P \in M} E^{P} f\left(S_{N}\right) .
$$

The inverse inequality follows from the representation (128) for any martingale measure being equivalent to the measure $P_{N}$.

\section{Vi. Models of Non-Arbitrage Incomplete Financial Markets}

Using the construction of the family of spot measures introduced in the previous section, this section presents the conditions under which the considered family of spot measures is invariant with respect to a certain class of evolutions of risky assets. For a certain class of contingent liabilities including a standard call option, the fair price of the super hedge is shown to be less than the spot price of the underlying asset. Specific applications of the results obtained for the previously known evolutions of risky assets are considered. New formulas are found for the nonarbitrage price range. A model of a non-arbitrage incomplete market is proposed and estimates are obtained in the case of a multi-parameter model of a non-arbitrage market.

On the probability space $\left\{\Omega_{N}, \mathcal{F}_{N}, P_{N}\right\}$, let us assume that the random values $b_{i}\left(\omega_{1}, \ldots, \omega_{i-1}\right), f_{i}\left(\omega_{1}, \ldots, \omega_{i}\right), \eta_{i}\left(\omega_{i}\right), i=\overline{1, N}$, satisfy the inequalities

$$
\begin{gathered}
b_{i}\left(\omega_{1}, \ldots, \omega_{i-1}\right)>0, \quad f_{i}\left(\omega_{1}, \ldots, \omega_{i}\right)>0, \\
\max _{\left\{\omega_{1}, \ldots, \omega_{i-1}\right\} \in \Omega_{i-1}} b_{i}\left(\omega_{1}, \ldots, \omega_{i-1}\right)< \\
\frac{1}{\max _{\left\{\omega_{1}, \ldots, \omega_{i-1}\right\} \in \Omega_{i-1}} \max _{\left\{\omega_{i}, \eta_{i}\left(\omega_{i}\right)<0\right\}} f_{i}\left(\omega_{1}, \ldots, \omega_{i}\right) \eta_{i}^{-}\left(\omega_{i}\right)}, \\
P_{i}^{0}\left(\eta_{i}\left(\omega_{i}\right)<0\right)>0, \quad P_{i}^{0}\left(\eta_{i}\left(\omega_{i}\right)>0\right)>0, \quad i=\overline{1, N} .
\end{gathered}
$$


As before, we put $\Omega_{i}^{0-}=\left\{\omega_{i} \in \Omega_{i}^{0}, \eta_{i}\left(\omega_{i}\right) \leq 0\right\}, \Omega_{i}^{0+}=\left\{\omega_{i} \in \Omega_{i}^{0}, \eta_{i}\left(\omega_{i}\right)>0\right\}$. We assume that the evolution $S_{n}\left(\omega_{1}, \ldots, \omega_{n}\right)$ of risky asset is given by the formula

$$
S_{n}\left(\omega_{1}, \ldots, \omega_{n}\right)=S_{0} \prod_{i=1}^{n}\left(1+b_{i}\left(\omega_{1}, \ldots, \omega_{i-1}\right) f_{i}\left(\omega_{1}, \ldots, \omega_{i}\right) \eta_{i}\left(\omega_{i}\right)\right), \quad n=\overline{1, N}
$$

With every point $v=\left\{\left(\omega_{1}^{1}, \omega_{1}^{2}\right), \ldots,\left(\omega_{N}^{1}, \omega_{N}^{2}\right)\right\} \in \mathcal{V}$, where $\mathcal{V}=\prod_{i=1}^{N}\left[\Omega_{i}^{0-} \times \Omega_{i}^{0+}\right]$, we connect the spot measure

$$
\begin{gathered}
\mu_{\left\{\omega_{1}^{1}, \omega_{1}^{2}\right\}, \ldots,\left\{\omega_{N}^{1}, \omega_{N}^{2}\right\}}(A)= \\
\sum_{i_{1}=1}^{2} \ldots \sum_{i_{N}=1}^{2} \prod_{n=1}^{N} \psi_{n}\left(\omega_{1}^{i_{1}}, \ldots, \omega_{n}^{i_{n}}\right) \chi_{A}\left(\omega_{1}^{i_{1}}, \ldots, \omega_{N}^{i_{N}}\right), \quad A \in \mathcal{F}_{N} .
\end{gathered}
$$

Let us denote $\nu_{v}(A)=\prod_{i=1}^{N} \nu_{\omega_{i}^{1}, \omega_{i}^{2}}\left(A_{i}\right), A=\prod_{i=1}^{N} A_{i}, \in \mathcal{F}_{N}$, the direct product of the measures $\nu_{\omega_{i}^{1}, \omega_{i}^{2}}\left(A_{i}\right), A_{i} \in \mathcal{F}_{i}^{0}, i=\overline{1, N}$, where $v=\left\{\left(\omega_{1}^{1}, \omega_{1}^{2}\right), \ldots,\left(\omega_{N}^{1}, \omega_{N}^{2}\right)\right\} \in \mathcal{V}$, $\mathcal{V}=\prod_{i=1}^{N}\left[\Omega_{i}^{0-} \times \Omega_{i}^{0+}\right]$, and

$$
\nu_{\omega_{i}^{1}, \omega_{i}^{2}}\left(A_{i}\right)=\chi_{A_{i}}\left(\omega_{i}^{1}\right) \frac{\eta_{i}^{+}\left(\omega_{i}^{2}\right)}{\eta_{i}^{-}\left(\omega_{i}^{1}\right)+\eta_{i}^{+}\left(\omega_{i}^{2}\right)}+\chi_{A_{i}}\left(\omega_{i}^{2}\right) \frac{\eta_{i}^{-}\left(\omega_{i}^{1}\right)}{\eta_{i}^{-}\left(\omega_{i}^{1}\right)+\eta_{i}^{+}\left(\omega_{i}^{2}\right)},
$$

for $\omega_{i}^{1} \in \Omega_{i}^{0-}, \omega_{i}^{2} \in \Omega_{i}^{0+}, A_{i} \in \mathcal{F}_{i}^{0}$. Then, there exists a countable additive function $\nu_{v}(A), A \in \mathcal{F}_{N}$, on the $\sigma$-algebra $\mathcal{F}_{N}$ for every $v \in \mathcal{V}$.

Theorem 12. On the probability space $\left\{\Omega_{N}, \mathcal{F}_{N}, P_{N}\right\}$, being the direct product of the probability spaces $\left\{\Omega_{i}^{0}, \mathcal{F}_{i}^{0}, P_{i}^{0}\right\}$, let the evolution of risky asset be given by the formula (135). For every point $v=\left\{\left(\omega_{1}^{1}, \omega_{1}^{2}\right), \ldots,\left(\omega_{N}^{1}, \omega_{N}^{2}\right)\right\} \in \mathcal{V}$, the spot measure $\mu_{\left\{\omega_{1}^{1}, \omega_{1}^{2}\right\}, \ldots,\left\{\omega_{N}^{1}, \omega_{N}^{2}\right\}}(A)$ given by the formula (136) does not depend on the random values $b_{i}\left(\omega_{1}, \ldots, \omega_{i-1}\right), i=\overline{1, N}$. In the case as $f_{i}\left(\omega_{1}, \ldots, \omega_{i}\right)=1, i=\overline{1, N}$, the formula

$$
\mu_{\left\{\omega_{1}^{1}, \omega_{1}^{2}\right\}, \ldots,\left\{\omega_{N}^{1}, \omega_{N}^{2}\right\}}(A)=\nu_{v}(A)
$$

is true. For the evolution of risky asset (135), the set of martingale measures being equivalent to the measure $P_{N}$ does not depend on the random values $b_{i}\left(\omega_{1}, \ldots, \omega_{i-1}\right), i=\overline{1, N}$.

Proof. Since the spot measures $\mu_{\left\{\omega_{1}^{1}, \omega_{1}^{2}\right\}, \ldots,\left\{\omega_{N}^{1}, \omega_{N}^{2}\right\}}(A)$ are given by the formula (136), to prove Theorem 12 it needs to prove that any $\psi_{n}\left(\omega_{1}, \ldots, \omega_{n}\right), n=\overline{1, N}$, does not depend on the random values $b_{i}\left(\omega_{1}, \ldots, \omega_{i-1}\right), i=\overline{1, N}$. Really,

$$
\begin{gathered}
\psi_{n}\left(\omega_{1}, \ldots, \omega_{n}\right)=\chi_{\Omega_{n}^{-}}\left(\omega_{1}, \ldots, \omega_{n-1}, \omega_{n}\right) \psi_{n}^{1}\left(\omega_{1}, \ldots, \omega_{n}\right)+ \\
\chi_{\Omega_{n}^{+}}\left(\omega_{1}, \ldots, \omega_{n-1}, \omega_{n}\right) \psi_{n}^{2}\left(\omega_{1}, \ldots, \omega_{n}\right) \\
\psi_{n}^{1}\left(\omega_{1}, \ldots, \omega_{n-1}, \omega_{n}\right)=
\end{gathered}
$$




$$
\begin{gathered}
\frac{\Delta S_{n}^{+}\left(\omega_{1}, \ldots, \omega_{n-1}, \omega_{n}^{2}\right)}{V_{n}\left(\omega_{1}, \ldots, \omega_{n-1}, \omega_{n}^{1}, \omega_{n}^{2}\right)}, \quad\left(\omega_{1}, \ldots, \omega_{n-1}\right) \in \Omega_{n-1}, \\
\psi_{n}^{2}\left(\omega_{1}, \ldots, \omega_{n-1}, \omega_{n}\right)= \\
\frac{\Delta S_{n}^{-}\left(\omega_{1}, \ldots, \omega_{n-1}, \omega_{n}^{1}\right)}{V_{n}\left(\omega_{1}, \ldots, \omega_{n-1}, \omega_{n}^{1}, \omega_{n}^{2}\right)}, \quad\left(\omega_{1}, \ldots, \omega_{n-1}\right) \in \Omega_{n-1} .
\end{gathered}
$$

But,

$$
\begin{gathered}
\Delta S_{n}^{+}\left(\omega_{1}, \ldots, \omega_{n-1}, \omega_{n}^{2}\right)= \\
S_{n-1}\left(\omega_{1}, \ldots, \omega_{n-1}\right) b_{n}\left(\omega_{1}, \ldots, \omega_{n-1}\right) f_{n}\left(\omega_{1}, \ldots, \omega_{n}^{2}\right) \eta_{n}^{+}\left(\omega_{n}^{2}\right), \\
\Delta S_{n}^{-}\left(\omega_{1}, \ldots, \omega_{n-1}, \omega_{n}^{1}\right)= \\
S_{n-1}\left(\omega_{1}, \ldots, \omega_{n-1}\right) b_{n}\left(\omega_{1}, \ldots, \omega_{n-1}\right) f_{n}\left(\omega_{1}, \ldots, \omega_{n}^{1}\right) \eta_{n}^{-}\left(\omega_{n}^{1}\right) .
\end{gathered}
$$

Therefore,

$$
\begin{gathered}
\frac{\Delta S_{n}^{+}\left(\omega_{1}, \ldots, \omega_{n-1}, \omega_{n}^{2}\right)}{V_{n}\left(\omega_{1}, \ldots, \omega_{n-1}, \omega_{n}^{1}, \omega_{n}^{2}\right)}= \\
\frac{f_{n}\left(\omega_{1}, \ldots, \omega_{n-1}, \omega_{n}^{2}\right) \eta_{n}^{+}\left(\omega_{n}^{2}\right)}{f_{n}\left(\omega_{1}, \ldots, \omega_{n-1}, \omega_{n}^{2}\right) \eta_{n}^{+}\left(\omega_{n}^{2}\right)+f_{n}\left(\omega_{1}, \ldots, \omega_{n-1}, \omega_{n}^{1}\right) \eta_{n}^{-}\left(\omega_{n}^{1}\right)}, \\
\frac{\Delta S_{n}^{-}\left(\omega_{1}, \ldots, \omega_{n-1}, \omega_{n}^{1}\right)}{V_{n}\left(\omega_{1}, \ldots, \omega_{n-1}, \omega_{n}^{1}, \omega_{n}^{2}\right)}= \\
\frac{f_{n}\left(\omega_{1}, \ldots, \omega_{n-1}, \omega_{n}^{1}\right) \eta_{n}^{-}\left(\omega_{n}^{1}\right)}{f_{n}\left(\omega_{1}, \ldots, \omega_{n-1}, \omega_{n}^{2}\right) \eta_{n}^{+}\left(\omega_{n}^{2}\right)+f_{n}\left(\omega_{1}, \ldots, \omega_{n-1}, \omega_{n}^{1}\right) \eta_{n}^{-}\left(\omega_{n}^{1}\right)}, \\
\left(\omega_{1}, \ldots, \omega_{n-1}\right) \in \Omega_{n-1} .
\end{gathered}
$$

From this, all the rest statements of Theorem 12 follow.

Theorem 13. On the probability space $\left\{\Omega_{N}, \mathcal{F}_{N}, P_{N}\right\}$, being the direct product of the probability spaces $\left\{\Omega_{i}^{0}, \mathcal{F}_{i}^{0}, P_{i}^{0}\right\}$, let the evolution of risky asset be given by the formula (135). Suppose that the nonnegative convex down payoff function $f(x)$ on the set $0 \leq x<\infty$ satisfies the inequality $0 \leq f(x)<x$. Then, the inequalities

$$
\begin{gathered}
f\left(S_{0}\right) \leq \sup _{P \in M} E^{P} f\left(S_{N}\right)= \\
\max _{\omega_{i}^{1} \in \Omega_{i}^{0-}, \omega_{i}^{2} \in \Omega_{i}^{0+}, i=\overline{1, N}} \int_{\Omega_{N}} f\left(S_{N}\right) d \mu_{\left\{\omega_{1}^{1}, \omega_{1}^{2}\right\}, \ldots,\left\{\omega_{N}^{1}, \omega_{N}^{2}\right\}}<S_{0}
\end{gathered}
$$

are true. 
Proof. Since the set of points $v=\left\{\left(\omega_{1}^{1}, \omega_{1}^{2}\right), \ldots,\left(\omega_{N}^{1}, \omega_{N}^{2}\right)\right\}$ in the set $\mathcal{V}$ is finite then the minimum in the formula

$$
\min _{\omega_{1}, \ldots, \omega_{N}}\left[S_{N}\left(\omega_{1}, \ldots, \omega_{N}\right)-f\left(S_{N}\left(\omega_{1}, \ldots, \omega_{N}\right)\right)\right]=d>0
$$

is reached at a certain point $v_{0}=\left\{\left(\omega_{1}^{1,0}, \omega_{1}^{2,0}\right), \ldots,\left(\omega_{N}^{1,0}, \omega_{N}^{2,0}\right)\right\}$. Therefore, the inequality

$$
S_{N}\left(\omega_{1}, \ldots, \omega_{N}\right)-f\left(S_{N}\left(\omega_{1}, \ldots, \omega_{N}\right)\right) \geq d, \quad\left\{\omega_{1}, \ldots, \omega_{N}\right\} \in \Omega_{N},
$$

is true

Integrating left and right parts of inequality over the measure $\mu_{\left\{\omega_{1}^{1}, \omega_{1}^{2}\right\}, \ldots,\left\{\omega_{N}^{1}, \omega_{N}^{2}\right\}}(A)$, we have

$$
\begin{gathered}
\int_{\Omega_{N}} S_{N}\left(\omega_{1}, \ldots, \omega_{N}\right) d \mu_{\left\{\omega_{1}^{1}, \omega_{1}^{2}\right\}, \ldots,\left\{\omega_{N}^{1}, \omega_{N}^{2}\right\}}- \\
\int_{\Omega_{N}} d \mu_{\left\{\omega_{1}^{1}, \omega_{1}^{2}\right\}, \ldots,\left\{\omega_{N}^{1}, \omega_{N}^{2}\right\}} f\left(S_{N}\left(\omega_{1}, \ldots, \omega_{N}\right)\right) \geq d .
\end{gathered}
$$

Since

$$
\int_{\Omega_{N}} S_{N}\left(\omega_{1}, \ldots, \omega_{N}\right) d \mu_{\left\{\omega_{1}^{1}, \omega_{1}^{2}\right\}, \ldots,\left\{\omega_{N}^{1}, \omega_{N}^{2}\right\}}=S_{0}
$$

we obtain the needed. It is evident that from the convexity down of payoff function $f(x)$ and Jensen inequality we obtain the inequality

$$
\int_{\Omega_{N}} f\left(S_{N}\left(\omega_{1}, \ldots, \omega_{N}\right)\right) d \mu_{\left\{\omega_{1}^{1}, \omega_{1}^{2}\right\}, \ldots,\left\{\omega_{N}^{1}, \omega_{N}^{2}\right\}} \geq f\left(S_{0}\right) .
$$

Theorem 13 is proved.

Let us note that the interval of non arbitrage prices for a certain processes was found in the papers [30], [31].

Corollary 2. For the standard call option of European type with payoff function $f(x)=(x-K)^{+}, K>0$, the conditions of Theorem 13 are true. Therefore, the inequalities (146) are valid.

Theorem 14. On the probability space $\left\{\Omega_{N}, \mathcal{F}_{N}, P_{N}\right\}$, being the direct product of the probability spaces $\left\{\Omega_{i}^{0}, \mathcal{F}_{i}^{0}, P_{i}^{0}\right\}$, let the evolution of risky asset be given by the formula (135). Suppose that the nonnegative convex down payoff function $f(x)$ on the set $0 \leq x<\infty$ satisfies the inequality $0 \leq f(x) \leq K, K>0$. Then, the inequalities

$$
f\left(S_{0}\right) \leq \sup _{P \in M} E^{P} f\left(S_{N}\right)=\max _{\omega_{i}^{1} \in \Omega_{i}^{0-}, \omega_{i}^{2} \in \Omega_{i}^{0+}, i=1, N} \int_{\Omega_{N}} f\left(S_{N}\right) d \mu_{\left\{\omega_{1}^{1}, \omega_{1}^{2}\right\}, \ldots,\left\{\omega_{N}^{1}, \omega_{N}^{2}\right\}} \leq K
$$

are true.

Proof. The proof is evident. 
Corollary 3. For the standard put option of European type with payoff function $f(x)=(K-x)^{+}, K>0$, the conditions of Theorem 14 are true. Therefore, the inequalities (152) are valid.

Corollary 4. For the standard call option of European type with payoff function $f(x)=(x-K)^{+}, K>0$, the interval of non arbitrage prices coincide with the interval

$$
\begin{gathered}
\left(\min _{\omega_{i}^{1} \in \Omega_{i}^{0-}, \omega_{i}^{2} \in \Omega_{i}^{0+}, i=\overline{1, N}} \int_{\Omega_{N}} f\left(S_{N}\right) d \mu_{\left\{\omega_{1}^{1}, \omega_{1}^{2}\right\}, \ldots,\left\{\omega_{N}^{1}, \omega_{N}^{2}\right\}},\right. \\
\left.\max _{\omega_{i}^{1} \in \Omega_{i}^{0-}{ }_{, \omega_{i}^{2} \in \Omega_{i}^{0+}, i=\overline{1, N}}} \int_{\Omega_{N}} f\left(S_{N}\right) d \mu_{\left\{\omega_{1}^{1}, \omega_{1}^{2}\right\}, \ldots,\left\{\omega_{N}^{1}, \omega_{N}^{2}\right\}}\right) .
\end{gathered}
$$

Corollary 5. For the standard put option of European type with payoff function $f(x)=(K-x)^{+}, K>0$, the interval of non arbitrage prices coincide with the interval

$$
\begin{gathered}
\left(\min _{\omega_{i}^{1} \in \Omega_{i}^{0-}, \omega_{i}^{2} \in \Omega_{i}^{0+}, i=\overline{1, N}} \int_{\Omega_{N}} f\left(S_{N}\right) d \mu_{\left\{\omega_{1}^{1}, \omega_{1}^{2}\right\}, \ldots,\left\{\omega_{N}^{1}, \omega_{N}^{2}\right\}},\right. \\
\left.\max _{\omega_{i}^{1} \in \Omega_{i}^{0-}{ }_{,}^{2} \in \Omega_{i}^{0+}, i=\overline{1, N}} \int_{\Omega_{N}} f\left(S_{N}\right) d \mu_{\left\{\omega_{1}^{1}, \omega_{1}^{2}\right\}, \ldots,\left\{\omega_{N}^{1}, \omega_{N}^{2}\right\}}\right) .
\end{gathered}
$$

Corollary 6. On the probability space $\left\{\Omega_{N}, \mathcal{F}_{N}, P_{N}\right\}$, being the direct product of the probability spaces $\left\{\Omega_{i}^{0}, \mathcal{F}_{i}^{0}, P_{i}^{0}\right\}$, let the evolution of risky asset is given by the formula

$$
S_{n}^{1}\left(\left(\omega_{1}, \ldots, \omega_{n}\right)=S_{0} \prod_{i=1}^{n}\left(1+\rho_{i}\left(\omega_{i}\right)\right), \quad n=\overline{1, N}\right.
$$

where the random value $\rho_{i}\left(\omega_{i}\right)$ is given on the probability space $\left\{\Omega_{i}^{0}, \mathcal{F}_{i}^{0}, P_{i}^{0}\right\}, i=$ $\overline{1, N}$, and let the bank account evolution be given by the formula

$$
B_{n}=B_{0}(1+r)^{n}, \quad r>0, \quad B_{0}>0, \quad n=\overline{1, N} .
$$

Then, for the discount evolution of risky asset

$$
S_{n}\left(\left(\omega_{1}, \ldots, \omega_{n}\right)=\frac{S_{0} \prod_{i=1}^{n}\left(1+\rho_{i}\left(\omega_{i}\right)\right)}{B_{0}(1+r)^{n}}, \quad n=\overline{1, N}\right.
$$

the set of martingale measure is nonempty one if the following conditions are true

$$
\begin{gathered}
P_{i}^{0}\left(\rho_{i}\left(\omega_{i}\right)-r<0\right)>0, P_{i}^{0}\left(\rho_{i}\left(\omega_{i}\right)-r>0\right)>0, \\
P_{i}^{0}\left(\rho_{i}\left(\omega_{i}\right)-r<0\right)+P_{i}^{0}\left(\rho_{i}\left(\omega_{i}\right)-r>0\right)=1, \quad i=\overline{1, N} .
\end{gathered}
$$


For every point $v=\left\{\left(\omega_{1}^{1}, \omega_{1}^{2}\right), \ldots,\left(\omega_{N}^{1}, \omega_{N}^{2}\right)\right\}$ in the set $\mathcal{V}$ the spot measure $\mu_{\left\{\omega_{1}^{1}, \omega_{1}^{2}\right\}, \ldots,\left\{\omega_{N}^{1}, \omega_{N}^{2}\right\}}(A)$ is a direct product of measures $\mu_{0}^{i}\left(A_{i}\right), A_{i} \in \mathcal{F}_{i}^{0}, i=\overline{1, N}$, given on the measurable space $\left\{\Omega_{i}^{0}, \mathcal{F}_{i}^{0}\right\}$, where $\mu_{0}^{i}\left(A_{i}\right)=\nu_{\omega_{i}^{1}, \omega_{i}^{2}}\left(A_{i}\right)$, and $\nu_{\omega_{i}^{1}, \omega_{i}^{2}}\left(A_{i}\right)$ is given by the formula (137) with $\eta_{i}\left(\omega_{i}\right)=\frac{\rho_{i}\left(\omega_{i}\right)-r}{1+r}, i=\overline{1, N}$. The fair price $\varphi_{0}$ of super-hedge of the nonnegative contingent liability $\varphi_{N}\left(\omega_{1}, \ldots, \omega_{N}\right)$ is given by the formula

$$
\varphi_{0}=\max _{v \in \mathcal{V}} \int_{\Omega_{N}} \varphi_{N}\left(\omega_{1}, \ldots, \omega_{N}\right) d \nu_{v}
$$

The interval of non-arbitrage prices is written in the form

$$
\left(\min _{v \in \mathcal{V}} \int_{\Omega_{N}} \varphi_{N}\left(\omega_{1}, \ldots, \omega_{N}\right) d \nu_{v}, \max _{v \in \mathcal{V}} \int_{\Omega_{N}} \varphi_{N}\left(\omega_{1}, \ldots, \omega_{N}\right) d \nu_{v}\right) .
$$

Theorem 15. On the probability space $\left\{\Omega_{N}, \mathcal{F}_{N}, P_{N}\right\}$ being the direct product of the probability spaces $\left\{\Omega_{i}^{0}, \mathcal{F}_{i}^{0}, P_{i}^{0}\right\}$, let the evolution of risky asset be given by the formula

$$
S_{n}^{1}\left(\left(\omega_{1}, \ldots, \omega_{n}\right)=S_{0} \prod_{i=1}^{n}\left(1+\rho_{i}\left(\omega_{i}\right)\right), \quad n=\overline{1, N}\right.
$$

where the random value $\rho_{i}\left(\omega_{i}\right)$, is given on the probability space $\left\{\Omega_{i}^{0}, \mathcal{F}_{i}^{0}, P_{i}^{0}\right\}$, $P_{i}^{0}\left(\left\{\rho_{i}\left(\omega_{i}\right)<0\right\}\right)>0, P_{i}^{0}\left(\left\{\rho_{i}\left(\omega_{i}\right)>0\right\}\right)>0, i=\overline{1, N}$, and let the bank account evolution be given by the formula

$$
B_{n}=B_{0} \prod_{i=1}^{n}\left(1+r_{i-1}\left(\omega_{i-1}\right)\right), \quad B_{0}>0, \quad n=\overline{1, N}
$$

where the strictly positive random values $r_{i}\left(\omega_{i}\right)$ are given on the probability $\left\{\Omega_{i}^{0}, \mathcal{F}_{i}^{0}, P_{i}^{0}\right\}, i=\overline{1, N}$. Then, for the discount evolution of risky asset

$$
S_{n}\left(\left(\omega_{1}, \ldots, \omega_{n}\right)=\frac{S_{0} \prod_{i=1}^{n}\left(1+\rho_{i}\left(\omega_{i}\right)\right)}{B_{0} \prod_{i=1}^{n}\left(1+r_{i-1}\left(\omega_{i-1}\right)\right)}, \quad n=\overline{1, N},\right.
$$

the set of martingale measure is nonempty one if the following conditions are true

$$
\begin{gathered}
\max _{\omega_{i-1} \in \Omega_{i-1}} r_{i-1}\left(\omega_{i-1}\right)<\min _{\omega_{i} \in \Omega_{i}, \rho_{i}\left(\omega_{i}\right)>0} \rho_{i}\left(\omega_{i}\right), \\
\min _{\omega_{i-1} \in \Omega_{i-1}} r_{i-1}\left(\omega_{i-1}\right)>0, i=\overline{2, N} \\
0<r_{0}<\min _{\omega_{1} \in \Omega_{1}, \rho_{1}\left(\omega_{1}\right)>0} \rho_{1}\left(\omega_{1}\right) .
\end{gathered}
$$

The fair price $\varphi_{0}$ of super-hedge of the nonnegative contingent liability $\varphi_{N}\left(\omega_{1}, \ldots, \omega_{N}\right)$ is given by the formula

$$
\varphi_{0}=\max _{\omega_{i}^{1} \in \Omega_{i}^{0-}, \omega_{i}^{2} \in \Omega_{i}^{0+}, i=1, N} \int_{\Omega_{N}} \varphi_{N}\left(\omega_{1}, \ldots, \omega_{N}\right) d \mu_{\left\{\omega_{1}^{1}, \omega_{1}^{2}\right\}, \ldots,\left\{\omega_{N}^{1}, \omega_{N}^{2}\right\}} .
$$


The interval of non-arbitrage prices is written in the form

$$
\begin{gathered}
\left(\min _{\omega_{i}^{1} \in \Omega_{i}^{0-}, \omega_{i}^{2} \in \Omega_{i}^{0+}, i=\overline{1, N}} \int_{\Omega_{N}} \varphi_{N}\left(\omega_{1}, \ldots, \omega_{N}\right) d \mu_{\left\{\omega_{1}^{1}, \omega_{1}^{2}\right\}, \ldots,\left\{\omega_{N}^{1}, \omega_{N}^{2}\right\}},\right. \\
\left.\max _{\omega_{i}^{1} \in \Omega_{i}^{0-},} \int_{\omega_{i}^{2} \in \Omega_{i}^{0+}, i=\overline{1, N}} \int_{\Omega_{N}} \varphi_{N}\left(\omega_{1}, \ldots, \omega_{N}\right) d \mu_{\left\{\omega_{1}^{1}, \omega_{1}^{2}\right\}, \ldots,\left\{\omega_{N}^{1}, \omega_{N}^{2}\right\}}\right) .
\end{gathered}
$$

Proof. The discount evolution (160) can be represented in the form

$$
\begin{gathered}
S_{n}\left(\omega_{1}, \ldots, \omega_{n}\right)= \\
\frac{S_{0}}{B_{0}}\left(1+\frac{\left(\rho_{1}\left(\omega_{1}\right)-r_{0}\right)}{1+r_{0}}\right) \prod_{i=2}^{n}\left(1+\frac{\rho_{i}\left(\omega_{i}\right)-r_{i-1}\left(\omega_{i-1}\right)}{1+r_{i-1}\left(\omega_{i-1}\right)}\right)= \\
\frac{S_{0}}{B_{0}} \prod_{i=1}^{n}\left(1+f_{i}\left(\omega_{1}, \ldots, \omega_{i}\right) \eta_{i}\left(\omega_{i}\right)\right),
\end{gathered}
$$

where

$$
\begin{gathered}
f_{1}\left(\omega_{1}\right)=\frac{1}{1+r_{0}}, \quad \eta_{1}\left(\omega_{1}\right)=\rho_{1}\left(\omega_{1}\right)-r_{0}, \\
f_{i}\left(\omega_{1}, \ldots, \omega_{i}\right)=\frac{\rho_{i}\left(\omega_{i}\right)-r_{i-1}\left(\omega_{i-1}\right)}{\left(\rho_{i}\left(\omega_{i}\right)-\min _{\omega_{i-1} \in \Omega_{i-1}} r_{i-1}\left(\omega_{i-1}\right)\left(1+r_{i-1}\left(\omega_{i-1}\right)\right)\right.}, \\
\eta_{i}\left(\omega_{i}\right)=\rho_{i}\left(\omega_{i}\right)-\min _{\omega_{i-1} \in \Omega_{i-1}} r_{i-1}\left(\omega_{i-1}\right) \quad i=\overline{2, N} .
\end{gathered}
$$

Since

$$
\begin{gathered}
f_{i}\left(\omega_{1}, \ldots, \omega_{i}\right)>0, \quad i=\overline{1, N} \\
P_{i}^{0}\left(\eta_{i}\left(\omega_{i}\right)<0\right)>0, \quad P_{i}^{0}\left(\eta_{i}\left(\omega_{i}\right)>0\right)>0, \quad i=\overline{1, N},
\end{gathered}
$$

then it means that the set of martingale measures being equivalent to $R_{N}$ is a nonempty set. Theorem 15 is proved.

Theorem 16. On the probability space $\left\{\Omega_{N}, \mathcal{F}_{N}, P_{N}\right\}$, being the direct product of the probability spaces $\left\{\Omega_{i}^{0}, \mathcal{F}_{i}^{0}, P_{i}^{0}\right\}$, let the evolution of risky asset be given by the formula

$$
S_{n}^{1}\left(\left(\omega_{1}, \ldots, \omega_{n}\right)=S_{0} \prod_{i=1}^{n} e^{\sigma_{i}\left(\omega_{1}, \ldots, \omega_{i-1}\right) \varepsilon_{i}\left(\omega_{i}\right)}, \quad n=\overline{1, N}\right.
$$

where the random values $\varepsilon_{i}\left(\omega_{i}\right), i=\overline{1, N}$, are such that 


$$
\begin{gathered}
P_{i}^{0}\left(\varepsilon_{i}\left(\omega_{i}\right)<0\right)>0, \quad P_{i}^{0}\left(\varepsilon_{i}\left(\omega_{i}\right)>0\right)>0, \\
P_{i}^{0}\left(\varepsilon_{i}\left(\omega_{i}\right)<0\right)+P_{i}^{0}\left(\varepsilon_{i}\left(\omega_{i}\right)>0\right)=1, \\
\sigma_{i}\left(\omega_{1}, \ldots, \omega_{i-1}\right) \geq \sigma_{i}^{0}>0, \quad i=\overline{1, N},
\end{gathered}
$$

and let the bank account evolution be given by the formula

$$
B_{n}=B_{0} \prod_{i=1}^{n}\left(1+r_{i-1}\left(\omega_{i-1}\right)\right), \quad B_{0}>0, \quad n=\overline{1, N}
$$

where the random values $r_{i}\left(\omega_{i}\right), i=\overline{1, N-1}$, are strictly positive ones, $r_{0}>0$. Then, for the discount evolution of risky asset

$$
S_{n}\left(\left(\omega_{1}, \ldots, \omega_{n}\right)=\frac{S_{0} \prod_{i=1}^{n} e^{\sigma_{i}\left(\omega_{1}, \ldots, \omega_{i-1}\right) \varepsilon_{i}\left(\omega_{i}\right)}}{B_{0} \prod_{i=1}^{n}\left(1+r_{i-1}\left(\omega_{i-1}\right)\right)}, \quad n=\overline{1, N}\right.
$$

the set of martingale measure is nonempty one, if

$$
\begin{gathered}
\exp \left\{\sigma_{1}^{0} \max _{\left\{\omega_{1}, \varepsilon_{1}\left(\omega_{1}\right)<0\right\}} \varepsilon_{1}\left(\omega_{1}\right)\right\}<r_{0}<\exp \left\{\sigma_{1}^{0} \min _{\left\{\omega_{1}, \varepsilon_{1}\left(\omega_{1}\right)>0\right\}} \varepsilon_{1}\left(\omega_{1}\right)\right\}, \\
\exp \left\{\sigma_{i}^{0} \max _{\left\{\omega_{i}, \varepsilon_{i}\left(\omega_{i}\right)<0\right\}} \varepsilon_{i}\left(\omega_{i}\right)\right\}<\min _{\left\{\omega_{i-1} \in \Omega_{i-1}\right\}} r_{i-1}\left(\omega_{i-1}\right)< \\
\max _{\left\{\omega_{i-1} \in \Omega_{i-1}\right\}} r_{i-1}\left(\omega_{i-1}\right)<\exp \left\{\sigma_{i}^{0} \min _{\left\{\omega_{i}, \varepsilon_{i}\left(\omega_{i}\right)>0\right\}} \varepsilon_{i}\left(\omega_{i}\right)\right\}, \quad i=\overline{2, N} .
\end{gathered}
$$

Then, the fair price of super-hedge $\varphi_{0}$ of the nonnegative contingent liability $\varphi_{N}\left(\omega_{1}, \ldots, \omega_{N}\right)$ is given by the formula

$$
\begin{gathered}
\varphi_{0}=\max _{v \in \mathcal{V}} \int_{\Omega_{N}} \varphi_{N}\left(\omega_{1}, \ldots, \omega_{N}\right) d \mu_{\left\{\omega_{1}^{1}, \omega_{1}^{2}\right\}, \ldots,\left\{\omega_{N}^{1}, \omega_{N}^{2}\right\}}= \\
\max _{v \in \mathcal{V}} \sum_{i_{1}=1}^{2} \ldots \sum_{i_{N}=1}^{2} \prod_{n=1}^{N} \psi_{n}\left(\omega_{1}^{i_{1}}, \ldots, \omega_{n}^{i_{n}}\right) \varphi_{N}\left(\omega_{1}^{i_{1}}, \ldots, \omega_{N}^{i_{N}}\right) .
\end{gathered}
$$

Proof. For the discount evolution (169), the following representation

$$
\begin{gathered}
S_{n}\left(\left(\omega_{1}, \ldots, \omega_{n}\right)=\right. \\
\frac{S_{0}}{B_{0}} \prod_{i=1}^{n}\left(1+f_{i}\left(\omega_{1}, \ldots, \omega_{i}\right) \eta_{i}\left(\omega_{i}\right)\right), \quad n=\overline{1, N}
\end{gathered}
$$


is true, where

$$
\begin{gathered}
\eta_{1}\left(\omega_{1}\right)=\exp \left\{\sigma_{1}^{0} \varepsilon_{1}\left(\omega_{1}\right)\right\}-r_{0}, \quad f_{1}\left(\omega_{1}\right)=\frac{1}{1+r_{0}}, \\
\eta_{i}\left(\omega_{i}\right)=\exp \left\{\sigma_{i}^{0} \varepsilon_{i}\left(\omega_{i}\right)\right\}-\max _{\left\{\omega_{i-1} \in \Omega_{i-1}\right\}} r_{i-1}\left(\omega_{i-1}\right), \\
f_{i}\left(\omega_{1}, \ldots, \omega_{i}\right)= \\
\frac{e^{\sigma_{i}\left(\omega_{1}, \ldots, \omega_{i-1}\right) \varepsilon_{i}\left(\omega_{i}\right)}-r_{i-1}\left(\omega_{i-1}\right)}{\left(\exp \left\{\sigma_{i}^{0} \varepsilon_{i}\left(\omega_{i}\right)\right\}-\max _{\left\{\omega_{i-1} \in \Omega_{i-1}\right\}} r_{i-1}\left(\omega_{i-1}\right)\right)\left(1+r_{i-1}\left(\omega_{i-1}\right)\right)}>0 \\
\left\{\omega_{1}, \ldots, \omega_{i}\right\} \in \Omega_{i}, \quad i=\overline{2, N}
\end{gathered}
$$

In this case, the spot measures

$$
\begin{gathered}
\mu_{\left\{\omega_{1}^{1}, \omega_{1}^{2}\right\}, \ldots,\left\{\omega_{N}^{1}, \omega_{N}^{2}\right\}}(A)= \\
\sum_{i_{1}=1}^{2} \ldots \sum_{i_{N}=1}^{2} \prod_{n=1}^{N} \psi_{n}\left(\omega_{1}^{i_{1}}, \ldots, \omega_{n}^{i_{n}}\right) \chi_{A}\left(\omega_{1}^{i_{1}}, \ldots, \omega_{N}^{i_{N}}\right), \quad A \in \mathcal{F}_{N},
\end{gathered}
$$

figuring in the formula (171), are determined by the formulas

$$
\begin{gathered}
\psi_{n}\left(\omega_{1}, \ldots, \omega_{n}\right)=\chi_{\Omega_{n}^{-}}\left(\omega_{1}, \ldots, \omega_{n-1}, \omega_{n}\right) \psi_{n}^{1}\left(\omega_{1}, \ldots, \omega_{n}\right)+ \\
\chi_{\Omega_{n}^{+}}\left(\omega_{1}, \ldots, \omega_{n-1}, \omega_{n}\right) \psi_{n}^{2}\left(\omega_{1}, \ldots, \omega_{n}\right) \\
\psi_{n}^{1}\left(\omega_{1}, \ldots, \omega_{n-1}, \omega_{n}\right)= \\
\frac{\Delta S_{n}^{+}\left(\omega_{1}, \ldots, \omega_{n-1}, \omega_{n}^{2}\right)}{V_{n}\left(\omega_{1}, \ldots, \omega_{n-1}, \omega_{n}^{1}, \omega_{n}^{2}\right)}, \quad\left(\omega_{1}, \ldots, \omega_{n-1}\right) \in \Omega_{n-1} \\
\psi_{n}^{2}\left(\omega_{1}, \ldots, \omega_{n-1}, \omega_{n}\right)= \\
\frac{\Delta S_{n}^{-}\left(\omega_{1}, \ldots, \omega_{n-1}, \omega_{n}^{1}\right)}{V_{n}\left(\omega_{1}, \ldots, \omega_{n-1}, \omega_{n}^{1}, \omega_{n}^{2}\right)}, \quad\left(\omega_{1}, \ldots, \omega_{n-1}\right) \in \Omega_{n-1}
\end{gathered}
$$

where

$$
\begin{gathered}
\frac{\Delta S_{n}^{+}\left(\omega_{1}, \ldots, \omega_{n-1}, \omega_{n}^{2}\right)}{V_{n}\left(\omega_{1}, \ldots, \omega_{n-1}, \omega_{n}^{1}, \omega_{n}^{2}\right)}= \\
\frac{f_{n}\left(\omega_{1}, \ldots, \omega_{n-1}, \omega_{n}^{2}\right) \eta_{n}^{+}\left(\omega_{n}^{2}\right)}{f_{n}\left(\omega_{1}, \ldots, \omega_{n-1}, \omega_{n}^{2}\right) \eta_{n}^{+}\left(\omega_{n}^{2}\right)+f_{n}\left(\omega_{1}, \ldots, \omega_{n-1}, \omega_{n}^{1}\right) \eta_{n}^{-}\left(\omega_{n}^{1}\right)}, \\
\frac{\Delta S_{n}^{-}\left(\omega_{1}, \ldots, \omega_{n-1}, \omega_{n}^{1}\right)}{V_{n}\left(\omega_{1}, \ldots, \omega_{n-1}, \omega_{n}^{1}, \omega_{n}^{2}\right)}=
\end{gathered}
$$




$$
\begin{gathered}
\frac{f_{n}\left(\omega_{1}, \ldots, \omega_{n-1}, \omega_{n}^{1}\right) \eta_{n}^{-}\left(\omega_{n}^{1}\right)}{f_{n}\left(\omega_{1}, \ldots, \omega_{n-1}, \omega_{n}^{2}\right) \eta_{n}^{+}\left(\omega_{n}^{2}\right)+f_{n}\left(\omega_{1}, \ldots, \omega_{n-1}, \omega_{n}^{1}\right) \eta_{n}^{-}\left(\omega_{n}^{1}\right)}, \\
\left(\omega_{1}, \ldots, \omega_{n-1}\right) \in \Omega_{n-1} .
\end{gathered}
$$

and the random values $\eta_{i}\left(\omega_{i}\right), f_{i}\left(\omega_{1}, \ldots, \omega_{i}\right), i=\overline{1, N}$, are given by the formulas (173). The obtained representation (172) proves Theorem 16.

Suppose that the random values $\eta_{k}\left(\omega_{k}\right), f_{k}\left(\omega_{1}, \ldots, \omega_{k}\right), k=\overline{1, N}$, and constants $a_{k}^{i}$ satisfy the inequalities

$$
\begin{gathered}
0<a_{k}^{i}<\frac{1}{\max _{\left\{\omega_{1}, \ldots, \omega_{k-1}\right\} \in \Omega_{k-1}} \max _{\left\{\omega_{k}, \eta_{k}\left(\omega_{k}\right)<0\right\}} f_{k}\left(\omega_{1}, \ldots, \omega_{k}\right) \eta_{k}^{-}\left(\omega_{k}\right)}, \quad k=\overline{1, N}, \quad i=\overline{1, d} \\
f_{i}\left(\omega_{1}, \ldots, \omega_{i}\right)>0, \quad P_{i}^{0}\left(\eta_{i}\left(\omega_{i}\right)<0\right)>0, \quad P_{i}^{0}\left(\eta_{i}\left(\omega_{i}\right)>0\right)>0, \quad i=\overline{1, N} .
\end{gathered}
$$

We assume that the evolutions of $d$ risky assets $S_{n}\left(\omega_{1}, \ldots, \omega_{n}\right)$ is given by the formula

$$
S_{n}\left(\omega_{1}, \ldots, \omega_{n}\right)=\left\{S_{n}^{i}\left(\omega_{1}, \ldots, \omega_{n}\right)\right\}_{i=1}^{d},
$$

where

$$
S_{n}^{i}\left(\omega_{1}, \ldots, \omega_{n}\right)=S_{0}^{i} \prod_{k=1}^{n}\left(1+a_{k}^{i} f_{k}\left(\omega_{1}, \ldots, \omega_{k}\right) \eta_{k}\left(\omega_{k}\right)\right), \quad n=\overline{1, N}, \quad i=\overline{1, d}
$$

Proposition 5. On the probability space $\left\{\Omega_{N}, \mathcal{F}_{N}, P_{N}\right\}$, being the direct product of the probability spaces $\left\{\Omega_{i}^{0}, \mathcal{F}_{i}^{0}, P_{i}^{0}\right\}$, let the evolution of risky assets be given by the formulas (181), (182), where the random values $\eta_{k}\left(\omega_{k}\right), f_{k}\left(\omega_{1}, \ldots, \omega_{k}\right)$ and constants $a_{k}^{i}, k=\overline{1, N}, i=\overline{1, d}$ satisfy the inequalities (180). For such an evolution of risky assets the set of martingale measures $\mu_{0}$ does not depend on $a_{k}^{i}$. The spot measures $\mu_{\left\{\omega_{1}^{1}, \omega_{1}^{2}\right\}, \ldots,\left\{\omega_{N}^{1}, \omega_{N}^{2}\right\}}(A)$ are determined by the formulas (174) - (179). The fair price $\varphi_{0}^{i}$ of super-hedge of the nonnegative contingent liability $\varphi_{N}^{i}\left(\omega_{1}, \ldots, \omega_{N}\right)$ is given by the formula

$$
\varphi_{0}^{i}=\max _{v \in \mathcal{V}} \int_{\Omega_{N}} \varphi_{N}^{i}\left(\omega_{1}, \ldots, \omega_{N}\right) d \mu_{\left\{\omega_{1}^{1}, \omega_{1}^{2}\right\}, \ldots,\left\{\omega_{N}^{1}, \omega_{N}^{2}\right\}}, \quad i=\overline{1, d}
$$

The interval of non-arbitrage prices is written in the form

$$
\begin{gathered}
\left(\min _{v \in \mathcal{V}} \int_{\Omega_{N}} \varphi_{N}^{i}\left(\omega_{1}, \ldots, \omega_{N}\right) d \mu_{\left\{\omega_{1}^{1}, \omega_{1}^{2}\right\}, \ldots,\left\{\omega_{N}^{1}, \omega_{N}^{2}\right\}},\right. \\
\left.\max _{v \in \mathcal{V}} \int_{\Omega_{N}} \varphi_{N}^{i}\left(\omega_{1}, \ldots, \omega_{N}\right) d \mu_{\left\{\omega_{1}^{1}, \omega_{1}^{2}\right\}, \ldots,\left\{\omega_{N}^{1}, \omega_{N}^{2}\right\}}\right), \quad i=\overline{1, d} .
\end{gathered}
$$


In the case $f_{k}\left(\omega_{1}, \ldots, \omega_{k}\right)=1, k=\overline{1, N}$, for every point $v=\left\{\left(\omega_{1}^{1}, \omega_{1}^{2}\right), \ldots,\left(\omega_{N}^{1}, \omega_{N}^{2}\right)\right\}$ in the set $\mathcal{V}$ the spot measure $\mu_{\left\{\omega_{1}^{1}, \omega_{1}^{2}\right\}, \ldots,\left\{\omega_{N}^{1}, \omega_{N}^{2}\right\}}(A)$ is a direct product of measures $\mu_{0}^{i}\left(A_{i}\right), A_{i} \in \mathcal{F}_{i}^{0}, i=\overline{1, N}$, given on the measurable space $\left\{\Omega_{i}^{0}, \mathcal{F}_{i}^{0}\right\}$, where $\mu_{0}^{i}\left(A_{i}\right)=$ $\nu_{\omega_{i}^{1}, \omega_{i}^{2}}\left(A_{i}\right)$, and $\nu_{\omega_{i}^{1}, \omega_{i}^{2}}\left(A_{i}\right)$ is given by the formula (137).

Suppose that $\left\{g_{k}^{i}\left(X_{N}\right)\right\}_{k=1}^{N}, i=\overline{1, d}$, are the mappings from the set $[0,1]^{N}$ into itself, where $X_{N}=\left\{x_{1}, \ldots, x_{N}\right\}, 0 \leq x_{k} \leq 1, k=\overline{1, N}$. If $S_{0}^{i}, S_{1}^{i}, \ldots, S_{N}^{i}, i=\overline{1, d}$, are the samples of the processes $(181)$, (182), let us denote the order statistics $S_{(0)}^{i}, S_{(1)}^{i}, \ldots, S_{(N)}^{i}, i=\overline{1, d}$, of this samples. Introduce also the denotation

$$
g_{k}^{i}\left(\left[S^{i}\right]_{N}\right)=g_{k}^{i}\left(\frac{S_{(0)}^{i}}{S_{(N)}^{i}}, \ldots, \frac{S_{(N-1)}^{i}}{S_{(N)}^{i}}\right), k=\overline{1, N}, i=\overline{1, d} .
$$

Let us introduce the denotations

$$
f_{k}^{1}=\max _{\left\{\omega_{1}, \ldots, \omega_{k-1}\right\} \in \Omega_{k-1}, \omega_{k}^{1} \in \Omega_{1}^{0-}} f_{k}\left(\omega_{1}, \ldots, \omega_{k-1}, \omega_{k}^{1}\right), k=\overline{1, N} .
$$

Proposition 6. Suppose that $S_{0}^{i}, S_{1}^{i}, \ldots, S_{N}^{i}$ is a sample of the random processes (181), (182). Then, for the parameters $a_{1}^{i}, \ldots, a_{N}^{i}$ the estimation

$$
\begin{gathered}
a_{1}^{i}=\frac{\left[1-\tau_{0}^{i} \frac{S_{(0)}^{i}}{S_{0}^{i}} g_{1}^{i}\left(\left[S^{i}\right]_{N}\right)\right]}{f_{1}^{1} \max _{\omega_{1}^{1} \in \Omega_{1}^{0-}} \eta_{1}^{-}\left(\omega_{1}^{1}\right)}, \quad 0<\tau_{0}^{i} \leq 1, \quad i=\overline{1, d}, \\
a_{k}^{i}=\frac{\left[1-\frac{g_{k}^{i}\left(\left[S^{i}\right]_{N}\right)}{g_{k-1}^{i}\left(\left[S^{i}\right]_{N}\right)}\right]}{f_{k}^{1} \max _{\omega_{k}^{1} \in \Omega_{k}^{0-}} \eta_{k}^{-}\left(\omega_{k}^{1}\right)}, \quad k=\overline{2, N}, \quad i=\overline{1, d},
\end{gathered}
$$

is valid, if for $g_{N}^{i}\left(\left[S^{i}\right]_{N}\right)>0, \quad\left[S^{i}\right]_{N} \in[0,1]^{N}$, the inequalities $g_{1}^{i}\left(\left[S^{i}\right]_{N}\right) \geq$ $g_{2}^{i}\left(\left[S^{i}\right]_{N}\right) \geq \ldots \geq g_{N}^{i}\left(\left[S^{i}\right]_{N}\right)$ are true. If $\tau_{0}^{i}=0$, then $a_{k}^{i}=1, k=\overline{1, N}, i=\overline{1, d}$.

\section{Vil. Applications}

In this section, we discuss the issue of applying the results obtained to real calculations of the range of non-arbitrage prices in the case of incomplete non-arbitrage markets. The first question that arises is what should be the evolution of risky assets when describing non-arbitrage markets. In this case, we must rely on the study of the evolution of stock index proposed in [22], [23], [24], that is,

$$
S_{n}\left(\omega_{1}, \ldots, \omega_{n}\right)=S_{0} \prod_{i=1}^{n} e^{\sigma_{i}\left(\omega_{1}, \ldots, \omega_{i-1}\right) \varepsilon_{i}\left(\omega_{i}\right)}, \quad n=\overline{1, N},
$$

where the random values $\sigma_{i}\left(\omega_{1}, \ldots, \omega_{i-1}\right) \geq \sigma_{i}^{0}>0, i=\overline{1, N}$, and $P_{i}^{0}\left(\varepsilon_{i}\left(\omega_{i}\right)<0\right)>$ $0, P_{i}^{0}\left(\varepsilon_{i}\left(\omega_{i}\right)>0\right)>0$, then such an evolution has the form (8) with

$$
f_{i}\left(\omega_{1}, \ldots, \omega_{i}\right)=\frac{e^{\sigma_{i}\left(\omega_{1}, \ldots, \omega_{i-1}\right) \varepsilon_{i}\left(\omega_{i}\right)}-1}{e^{\sigma_{i}^{0} \varepsilon_{i}\left(\omega_{i}\right)}-1}, \quad \eta_{i}\left(\omega_{i}\right)=e^{\sigma_{i}^{0} \varepsilon_{i}\left(\omega_{i}\right)}-1, \quad i=\overline{1, N} .
$$

satisfying needed conditions. Here, the random values $\sigma_{i}\left(\omega_{1}, \ldots, \omega_{i-1}\right), i=\overline{1, N}$, are conditional volatilities, $\varepsilon_{i}\left(\omega_{i}\right), i=\overline{1, N}$, are identically distributed random values. 
Therefore, when modeling non-arbitrage securities markets, the evolution of the stock index should be described by formula (167). The evolution of shares quoted on the exchange should be described by parametric processes described by formulas (181), (182). The parameters of such a process are determined in accordance with the Proposition 6.

\section{CONCLUSIONS}

Section 3 contains the results related to the uniqueness of the set of martingale measures. In Lemma 1 it is shown that in the case of evolution of risky assets given by formula (11) there is only one spot martingale measure for the considered class of risky assets. A wide class of risky asset evolutions has been identified for modeling real processes in the financial market. In Theorem 1, necessary and sufficient conditions are given for the evolution of risky assets under which the martingale measure is the only one, and in Theorem 2 it is shown that it coincides with a point martingale measure.

In section 4, Proposition 1 formulates the conditions for the evolution of risky assets under which the martingale measure is the same for a wide class of evolutions of risky assets. Proposition 2 states that the considered securities market in Proposition 1 is complete and non-arbitrage and provides formulas for the fair values of the contingent liabilities.

A direct consequence of the considered results is Corollary 1 known as the Cox, Ross, Rubinstein model and Theorem 3 being the direct generalization of the above mentioned model. In Theorem 4, the conditions are found under which the discounted evolution can be represented in the form considered in the work. A formula is found for the fair price of the super-hedge in this realistic case. In Proposition 3, a parametric model of the complete non-arbitrage market is proposed and formulas for the fair prices of contingent liabilities are written out. Proposition 4 provides an assessment of the parameters of a complete non-arbitrage market model, which opens up opportunities for modeling processes in financial markets.

Section 5 presents the theoretical foundations of the incomplete non-arbitrage market model. In Lemmas 2 and 3, conditions for the evolution of risky assets are formulated for which the family of martingale measures is equivalent to the original one. It is shown in Theorem 5 that the family of measures constructed in Lemma 2 is a family of martingale measures equivalent to the original measure. In Lemma 4 and Theorems 6 and 7, estimates are found for nonnegative random variables that ensure the validity of the optional decomposition for nonnegative super-martingales with respect to all martingale measures presented in Theorem 8. In contrast to earlier results, the optional decomposition can be found explicitly here. Lemma 5 contains a result that introduced in Definition 1 the spot measure is a martingale one (see also in [1]).

Theorems 9 and 10 describe all martingale measures equivalent to the original measure. In the case under consideration, the conditions of Theorems 9 and 10 are not restrictive. In Theorem 11, a formula is found for the fair price of the superhedge for random claims, which allows it to be calculated using a finite number of operations.

Section 6 presents possible models of incomplete non-arbitrage markets. For this, Theorem 12 shows that the set of spot measures does not depend on a certain type of evolution of risky assets and is one and the same set. Under certain simplified conditions, each spot measure is a direct product of the spot measures indicated in the theorem. Due to the finiteness of the set of spot measures in Theorem 13, it was found that for a certain class of contingent liabilities the super-hedge price is less than the initial price of the underlying asset. The range of non-arbitrage prices is found. Among these contingent liabilities is the standard European call option. Non-arbitrage price interval is found. Corollaries 6 and 15 provide examples of the evolution of risky assets. 
Theorems 15 and 16 consider realistic models of the evolution of both risky and non-risky assets for which there is a finite family of point measures.

Proposition 5 presents a realistic parametric model of an incomplete nonarbitrage market and also presents formulas for the fair price of the super hedge and the range of non-arbitrage prices. In Proposition 6, estimates of the parameters of the incomplete non-arbitrage market model are found.

\section{References Références Referencias}

1. Gonchar, N. S. (2020) Derivatives pricing in Non-arbitrage market, Global Journal of Science Frontier Research, V.20 issue 14, P. 32-101.

2. Gonchar, N. S. (2001) Fund market and economic grows. Bogolyubov Institute for Theoretical Physics, Kiev (in Ukrainian).

3. Cox J., Ross R.A., Rubinstein M. (1976) Option pricing: a simplied approach, Journal of Financial Economics, V. 7, P. 229 - 263.

4. Bachelier L.(1900) Theorie de la speculation. Annales de l'Ecole Normal Superieure. V. 17, P. 21-86.

5. Black F., Scholes M. (1973) The pricing of options and corporate liabilities. Journal of Political Economy, V. 81, N 3, P. 637 - 659.

6. Merton R. S. (1973) Theory of rational option pricing. Bell Journal of Economics and Management Science. N 4 (Spring), P. 141 - 183.

7. Harrison J.M., Kreps D.M. (1979) Martingales and Arbitrage in Multiperiod Securities Markets. Journal of Economic Theory, 20, 381- 408. https://doi.org/10.1016/0022-0531(79)90043-?

8. Harrison J.M., Pliska S.R. (1981) Martingales and Stochastic Integrals in the Theory of Continuous Trading. Stochastic Processes and their Applications. 11, 215.

9. Dalang R.C., Morton A., Willinger W. (1990): Equivalent Martingale measures and no-arbitrage in stochastic securities market model. Stochastics and Stochastic Reports. 29, 185-201. https://doi.org/10.1080/17442 509008833613

10. Schachermayer, W. (2003): Introduction to the Mathematics of Financial Markets. In: S. Albeverio, W. Schachermayer, M. Talagrand: Springer Lecture Notes in Mathematics 1816 - Lectures on Probability Theory and Statistics, Saint-Flour summer school 2000 (Pierre Bernard, editor), Springer Verlag, Hei-delberg, pp. 111-177.

11. Delbaen, F., and Schachermayer, W.(2006): The Mathematics and Arbitrage. Berlin: Springer.

12. Rogers L.C. G. (1995) Equivalent martingale measures and no-arbitrage. Stochastics and Stochastics Reports, V. 51, P. 41 - 50.

13. Shiryaev A. N. (1998) Foundations of Stochastic Financial Mathematics. V.1 Theory. PHAZIS, Moskow (in Russian).

14. Shiryaev A. N. (1998) Foundations of Stochastic Financial Mathematics. V.2 Theory. PHAZIS, Moskow (in Russian).

15. Gonchar, N. S. (2008) Mathematical foundations of information economics. Bogolyubov Institute for Theoretical Physics, Kiev.

16. Gonchar, N. S. (2015): Mathematical Model of Banking Operation. Cybernetics and System Analysis, 51, 378-399. https://doi.org/ 10.1007/s 10559-015-9730-0

17. Gonchar, N. S., and Terentieva, L.S.(2008) Default Risk Valuation of the Firm with the Special Process of Internal Yield. Journal of Automation and Information Sciences, 40, 57-71. https://doi.org/ 10.1615/ AutomatInfScien. $\mathrm{v} 40.18 .60$

18. Gonchar N.S. (2017) Banking and Risk Assessment. In: Jerzy, K., Ed., Banking: Services, Opportunity and Risks, Chapter 8, Nova Science Publisher, Inc., New York. 
19. Gonchar N.S. (2020): Assessment of contingent liabilities for risk assets evolutions built on Brownian motion. Advances in Pure Mathematics, 9, 259 - 296. https://doi.org/10.4236/apm.2020.105016

20. Gonchar N.S. (2019): Description of incomplete financial markets for time evolution of risk assets. Advances in Pure Mathematics, 10, 567 - 610. https://doi.org/10.4236/apm.2019.96029

21. Gonchar N.S. (2018): Martingales and super-martingales relative to a convex set of equivalent measures. Advances in Pure Mathematics, 8, 428 462. https://doi.org/10.4236/apm.2018.84025

22. Engle R. F. (1982) Autoregressive conditional heteroscedasticity with estimates of the variance of United Kingdom ination. Econometrica V. 50, N 4, P 987-1008.

23. Bollerslev T. (1986) Generalized autoregressive conditional heteroskedasticity. Journal of Econometrics, V. 31, P. 307-327.

24. Engle R. F., Bollerslev T. (1986) Moddeling the persistence of conditional variance. Econometrics Reviews, V. 5, P. 1-50.

25. El Karoui, N., and Quenez, M.C. (1995): Dynamic programming and pricing of contingent claims in an incomplete market. SIAM J. Control Optimizat., $33,27-66$.

26. Kramkov, D. O. (1996) Optional decomposition of super-martingales and hedging in incomplete security markets. Probab. Theory Relat. Fields, 105, 459-479. https://doi.org/ 10.1007/BF01191909

27. Follmer, H., and Kramkov, D.O. (1997): Optional decomposition theorem under constraints. Probability Theory and Related Fields, 109, 1-25. https://doi.org/10.1007/s004400050122

28. Follmer, H., and Kabanov, Yu. M.(1996): 'Optional decomposition theorems in discrete time,' in Atti del convegno in onore di Oliviero Lessi, Padova, 25-26 marzo, 47-68.

29. Follmer, H., and Kabanov, Yu. M. (1998): Optional decomposition and Lagrange multipliers. Finance Stochast., 2, 69-81.https://doi.org/10.1007/s 007800050033

30. Eberlein, E., Jacod, J. (1997): On the range of option price. Finance Stoch. 1.

31. Bellamy, N., Jeanblanc, M. (1999): Incompleteness of markets driven by mixed diffusion. Finance Stoch. 4, 209 - 222. 